\title{
THE CHECKLIST OF HUNGARIAN BRYOPHYTES - SECOND UPDATE
}

\author{
Peter Erzberger ${ }^{1}$ and Beáta PAPP ${ }^{2}$ \\ ${ }^{1}$ Belziger Str.37,D-10823 Berlin, Germany; erzberger.peter@gmail.com \\ ${ }^{2}$ Department of Botany, Hungarian Natural History Museum, H-1431 Budapest, Pf. 137, Hungary
}

Erzberger, P. \& Papp, B. (2020): The checklist of Hungarian bryophytes - second update. - Studia bot. hung. 51(2): 11-76.

\begin{abstract}
According to the checklist presented here, the bryophyte flora of Hungary consists of 2 species in 2 genera and 2 families of hornworts, 146 species and 3 additional infraspecific taxa ( 1 subspecies and 2 varieties) in 60 genera and 34 families of liverworts, and 521 species and 17 additional infraspecific taxa ( 5 subspecies and 12 varieties) in 186 genera and 64 families of mosses, in total 669 species, 6 subspecies and 14 varieties or 689 taxa. During the last decade, 40 bryophyte taxa (6 liverworts and 34 mosses) were added to the Hungarian bryophyte flora (Appendix 1.1), and an additional 19 taxa (4 liverworts and 15 mosses) that were already part of the Hungarian flora but not recognised in the previous checklist, are now incorporated in the list (Appendix 1.2). On the other hand, 29 taxa (7 liverworts and 22 mosses) must be excluded according to our present state of knowledge (Appendix 2). Another 13 taxa (2 liverworts and 11 mosses) that are at present not considered members of the Hungarian bryoflora are annotated in Appendix 3. They were not recognised in the previous checklist (with one exception), but are reported from Hungary in some literature and with five exceptions are also recognised in the European checklist. In many cases we have insufficient information to definitely decide upon their status. Annotations to all taxa listed in the three appendices provide additional information. In Appendix 4 to all names of bryophyte taxa that appeared in the previous checklist (except excluded taxa) the presently accepted names are assigned.
\end{abstract}

Key words: hornworts, liverworts, mosses, nomenclature, synonymy, taxonomy

\section{INTRODUCTION}

The first checklist of the bryophytes of Hungary (ERzBERGER and PAPP 2004) intended to adopt moderately modern taxonomy to the Hungarian bryophyte flora which had been summarised in three major accounts during the 20th century (BOROs 1953, 1968, ORBÁN and VAJDA 1983). These, however, were rather outdated by the time. Also, many reports of species not mentioned in these accounts and published in a scattered literature, were scrutinised and added to the list if approved. The updated list (PAPP et al. 2010), at the same time the first red list, was based on ERZBERGER and PAPP (2004) as far as taxonomy and nomenclature is concerned, but incorporated 30 additional taxa, many recognised during 
herbarium revisions dealing with the legacy of Boros and Vajda (ERZBERGER and SCHRÖDER 2008, ERZBERGER 2009a), and more recent fieldwork. Meanwhile, another 40 taxa were added to the Hungarian bryophyte flora, and taxonomy has undergone dramatic changes, mainly due to molecular work, resulting also in a bewildering number of nomenclatural substitutions. The time has therefore come to present a second update of the checklist. An update of the red list is also urgently needed, since we now have a much better knowledge of the situation of many species, but to evaluate this, more time will be needed. On a European scale, a new red list (HodGetTs et al. 2019) and a new checklist (HoDGETTS et al. 2020) have recently been published which are the result of coordinating the work of many individual authors (94 in HodgetTs et al.2019, 26 in HodgetTs et al.2020) and thus setting new standards. It seems a good occasion to follow the new European checklist with respect to taxonomy as far as possible, since a uniform approach will create best comparability between European countries.

Whereas the first Hungarian checklist (ERzBERGER and PAPP 2004) commented on many infraspecific taxa, these were nearly completely omitted in the updated list (PAPP et al.2010). However, the new European checklist (HodGETTS et al.2020) draws attention to many subspecies and varieties, some of which were recognised in Hungary in the 20th century (literature reports or herbarium specimens) or found during recent field work; these have been incorporated into the list when appropriate.

Finally, 29 taxa should be excluded from the Hungarian bryoflora, mostly since their vouchers turned out to represent other taxa (Appendix 2).

We follow the nomenclature of HodgETTs et al. (2020) with certain reservations. Any nomenclatural change should ultimately aim at nomenclatural stability. However, obviously, the present stage of molecular work is not final and therefore taxonomy as well as nomenclature will doubtless continue to change even in the near future.

On the other hand, the checklist, and in particular the future red list, which most likely will be largely based on the former regarding taxonomy and nomenclature, will be read and used also by many non-experts, e.g. professionals and national park staff involved with conservational aspects. For these users, the 168 nomenclatural novelties will be cumbersome, and make the necessary comparison with accounts published earlier very troublesome. To ensure compatibility with previous publications (and the extensive list of synonymies referring to the work of Boros, Orbán and Vajda published in ERZBERGER and PAPP 2004) we therefore decided to present our updated checklist Janus-faced, including an abridged version of the present checklist (without the newly added and excluded taxa) using the 'old' nomenclature with reference to the new names (Appendix 4). 


\section{METHODS}

The taxa listed in Hodgetts et al. (2020) were compared with those reported from Hungary in ERzBerger and PAPP (2004), PAPP et al. (2010) and other publications from the period 2010-2019 concerning the Hungarian bryoflora (most referenced in NAGY et al. 2019). In few cases unpublished results were also considered. Whenever specimen details are given, the code of the quadrant in the Central European system (BARTH A et al. 2015) is given in square brackets. Separate lists of recognised taxa, taxa not listed in PAPP et al. (2010) (Appendix 1 ), excluded taxa (Appendix 2), and problematic taxa (Appendix 3) were compiled. Nomenclature in general follows Hodgets et al. (2020) with the following exceptions: Bryum barnesii, B. bicolor, B. stirtonii, Hypnum pallescens var. reptile, and Microbryum muticum are not recognised in HodgetTs et al. (2020). For details, see the annotations to these taxa. On the other hand, we do not differentiate between Riccia canaliculata and $R$. duplex, nor between the two subspecies of Fissidens crassipes (ERzBER GER 2016b). We also include Brachythecium tenuicaule in the synonymy of $B$. tommasinii.

\section{RESULTS}

\section{Annotated and updated checklist of Hungarian bryophytes}

Nomenclature and taxonomy mostly according to HodgetTs et al. 2020) ordered alphabetically according to the accepted names (for comparison with older literature, see Appendix 4, where ordering is according to the names in older checklists (ERzBERgER and PAPP 2004, PAPP et al. 2010), and thus synonymy can be found. Synonyms from HodGETTs et al. (2020) are in square brackets. If the name used in PAPP et al. (2010) is not among them, it is added in parentheses (irrespective to its taxonomic correctness). Annotations in Hodget $e$ et al. (2020) mostly highlight molecular evidence for taxonomic placement, these are generally not repeated here. Our annotations mostly deal with the reports for the occurrence of taxa in Hungary, but also discuss some taxonomic questions and cases where we do not follow the nomenclature of the European checklist.

\section{Hornworts}

Anthoceros agrestis Paton [Anthoceros punctatus subsp. agrestis (Paton) Damsh., Anthoceros punctatus var. douinii (R. M. Schust.) Damsh.]

Phaeoceros carolinianus (Michx.) Prosk. [Phaeoceros laevis subsp. carolinianus (Michx.) Prosk.] 


\section{Liverworts}

Anastrophyllum michauxii (F. Weber) H. Buch

Aneura pinguis (L.) Dumort. [Aneura pinguis var. angustior (Hook.) Dumort., Aneura pinguis var. denticulata (Nees) Godelin., Aneura pinguis var. fuscovirens (Lindb.) Damsh.]

Apopellia endiviffolia (Dicks.) Nebel et D. Quandt [Pellia endiviifolia (Dicks.) Dumort.]

Asterella saccata (Wahlenb.) A. Evans

Barbilophozia barbata (Schmidel ex Schreb.) Loeske [Lophozia barbata (Schmidel ex Schreb.) Dumort.]

Barbilophozia hatcheri (A. Evans) Loeske [Lophozia hatcheri (A. Evans) Steph.] - (NÉmetH and NAgY 2016).

Barbilophozia sudetica (Nees ex Huebener) L. Söderstr., De Roo et Hedd. [Lophozia alpestris auct. non (Schleich. ex F. Weber) A. Evans, L. sudetica (Nees ex Huebener) Grolle, Pseudolophozia sudetica (Nees ex Huebener) Konstant. et Vilnet, Lophozia sudetica var. anomala (Schljakov) Schljakov, L. debiliformis R. M. Schust. et Damsh., Pseudolophozia debiliformis (R. M. Schust. et Damsh.) Konstant. et Vilnet, Lophozia debiliformis var. concolor R. M. Schust. et Damsh.] Bazzania trilobata (L.) Gray

Blasia pusilla L.

Blepharostoma trichophyllum (L.) Dumort.

Calypogeia azurea Stotler et Crotz

Calypogeia fissa (L.) Raddi

Calypogeia integristipula Steph.

Calypogeia muelleriana (Schiffn.) Müll. Frib.

Calypogeia neesiana (C. Massal. et Carestia) Müll. Frib.

Calypogeia suecica (Arnell et J. Perss.) Müll. Frib.

Cephalozia bicuspidata (L.) Dumort.

Cephalozia lacinulata (J. B. Jack ex Gottsche et Rabenh.) Spruce

Cephaloziella divaricata (Sm.) Schiffn. var. divaricata

Cephaloziella divaricata (Sm.) Schiffn. var. scabra (M. Howe) Haynes [Cephaloziella divaricata var. asperifolia (Taylor) Damsh.] - According to HodgetTs et al. (2020), the taxonomic value of Cephaloziella divaricata var. scabra is controversial. In Central Europe var. scabra intergrades with var. divaricata and is possibly only a morph of shady habitats (KöCKINGER 2017). Occurrence in Hungary (unpublished): [9975.1] Baranya County, Mecsek, PécsÜrög, Éger-völgy, N $46^{\circ} 05^{\prime} 31.3^{\prime \prime}$, E $18^{\circ} 10^{\prime} 26.0$ ”, acidophilous oak forest, soil over sandstone, 360 m a.s.l., 07.10.2014, leg. Erzberger, Csiky, Kovács, det. Meinunger (B-Erzberger 19052). 
Cephaloziella hampeana (Nees) Schiffn. ex Loeske [Cephaloziella hampeana var. subtilis (Velen.) Macvicar]

Cephaloziella integerrima (Lindb.) Warnst. [Dichiton integerrimum (Lindb.) H. Buch, Cephaloziella integerrima var. obtusa Müll. Frib.]

Cephaloziella rubella (Nees) Warnst. [Cephaloziella rubella var. sullivantii (Austin) Müll. Frib. ex R. M. Schust., Cephaloziella rubella var. bifida (Lindb.) Douin, Cephaloziella rubella var. pulchella (C. E. O. Jensen) R. M. Schust.]

Cephaloziella spinigera (Lindb.) Jørg. [Cephaloziella spinigera f. striatula (C. E. O. Jensen) Damsh., Cephaloziella subdentata Warnst.]

Cephaloziella stellulifera (Taylor ex Carrington et Pearson) Croz. [Cephaloziella stellulifera var. limprichtii (Warnst.) Macvicar]

Cephaloziella varians (Gottsche) Steph. [Cephaloziella alpina Douin, Cephaloziella arctica Bryhn et Douin, Cephaloziella varians var. arctica (Bryhn et Douin) Damsh., Cephaloziella varians var. scabra (S. W. Arnell) Damsh.] ERZBERGER and MEINUNGER (2014a).

Chiloscyphus pallescens (Ehrh.) Dumort.

Chiloscyphuspolyanthos (L.) Corda

Clevea hyalina (Sommerf.) Lindb. [Athalamia hyalina (Sommerf.) S. Hatt., Clevea byalina var. suecica (Lindb.) Lindb., Athalamia hyalina var. suecica (Lindb.)

S. Hatt., Clevea hyalina var. rufescens (S. W. Arnell) Konstant. nom. inval.]

Cololejeunea calcarea (Lib.) Steph.

Cololejeunea rossettiana (C. Massal.) Schiffn.

Conocephalum conicum (L.) Dumort.

Conocephalum salebrosum Szweyk., Buczk. et Odrzyk.

Crossocalyx bellerianus (Nees ex Lindenb.) Meyl. [Anastrophyllum hellerianum (Nees ex Lindenb.) R. M. Schust.]

Diplophyllum albicans (L.) Dumort.

Diplophyllum obtusifolium (Hook.) Dumort.

Endogemma caespiticia (Lindenb.) Konstant., Vilnet et A. V. Troitsky [Jungermannia caespiticia Lindenb.]

Fossombronia foveolata Lindb.

Fossombronia pusilla (L.) Nees

Fossombronia wondraczekii (Corda) Dumort. ex Lindb.

Frullania cleistostoma Schiffn. et W. Wollny [Frullania inflata auct. eur. non Gottsche]

Frullania dilatata (L.) Dumort.

Frullania fragilifolia (Taylor) Gottsche, Lindenb. et Nees

Frullania jackii Gottsche [Frullania davurica subsp. jackii (Gottsche) S. Hatt.]

Frullania tamarisci (L.) Dumort. [Frullania tamarisci var. atrovirens Carrington, Frullania tamarisci var. cornubica Carrington, Frullania tamarisci var. erice- 
torum Jørg., Frullania tamarisci var. robusta Lindb., Frullania tamarisci var. sardoa (De Not.) De Not.]

Fuscocephaloziopsis catenulata (Huebener) Váňa et L. Söderstr. [Cephalozia catenulata (Huebener) Lindb., Pleurocladula catenulata (Huebener) Konstant., Vilnet et Troitsky]

Fuscocephaloziopsis lunulifolia (Dumort.) Váňa et L. Söderstr. [Cephalozia lunulifolia (Dumort.) Dumort., Pleurocladula lunulifolia (Dumort.) Konstant., Vilnet et Troitsky]

Fuscocephaloziopsis macrostachya (Kaal.) Váňa et L. Söderstr. [Cephalozia macrostachya Kaal., Pleurocladula macrostachya (Kaal.) Konstant., Vilnet et Troitsky]

Fuscocephaloziopsis pleniceps (Austin) Váňa et L. Söderstr. [Cephalozia pleniceps (Austin) Lindb., Pleurocladula pleniceps (Austin) Konstant., Vilnet et Troitsky]

Gymnocolea inflata (Huds.) Dumort.

Isopaches bicrenatus (Schmidel ex Hoffm.) H. Buch [Lophozia bicrenata (Schmidel ex Hoffm.) Dumort.]

Jungermannia atrovirens Dumort. [Jungermannia lanceolata L. nom. rejic., Jungermannia lanceolata var. atrovirens (Dumort.) Damsh.]

Jungermannia pumila With. [Jungermannia pumila var. alpestris Gottsche et Rabenh.]

Lejeunea cavifolia (Ehrh.) Lindb.

Lepidozia reptans (L.) Dumort. [Lepidozia reptans var. julacea (Nees) Damsh. nom. inval., Lepidozia reptans var. tenera (Huebener) P. Allorge nom. inval.]

Liochlaena lanceolata Nees [Jungermannia lanceolata auct. non L., Jungermannia leiantha Grolle, Jungermannia subulata var. leiantha (Grolle) Damsh.]

Liochlaena subulata (A. Evans) Schljakov [Jungermannia subulata A. Evans]

Lophocolea coadunata (Sw.) Mont. [Chiloscyphus coadunatus (Nees) J. J. Engel et R. M. Schust., Lophocolea cuspidata auct. non (Nees) Limpr., L. bidentata var. rivularis (Raddi) Schiffn., Chiloscyphus latifolius (Nees) J. J. Engel et R. M. Schust.] - See annotation 84 in HodgetTs et al. (2020): 'The Lophocolea bidentata complex has been treated in various ways in the past, and there is also some nomenclatural confusion. VÁŇA and ENGEL (2013) clarify the concepts, not only for Europe but world-wide. As we follow the World checklist (SöDERSTRÖM et al. 2016), we here treat them in the genus Lophocolea as Lophocolea bidentata (autoicious) and Lophocolea coadunata (dioicious). Many earlier reports are confusing and the occurrences in some countries are still questioned.' Vegetative distinguishing characters are mostly not diagnostic. On the other hand, the formation of antheridia and archegonia seems to be influenced by environmental factors, and in most cases it is impossible to unequivocally 
determine the sexual condition (MeINUNGer and SCHRöDER 2007). Many authors therefore treat the complex as a single, variable species. According to KöCKINGER (2017), the dioicous L. coadunata is frequent, whereas the autoicous $L$. bidentata is rare. We tentatively identify the frequent taxon occurring in Hungary as the dioicous L. coadunata, and exclude L. bidentata. At present, we refrain from rectifying reports in Boros (1968) and ORBÁN and VAJDA (1983) of Lophocolea cuspidata (Nees) Limpr. and L. alata Mitt.

Lophocolea heterophylla (Schrad.) Dumort. [Chiloscyphus profundus (Nees) J. J. Engel et R. M. Schust.]

Lophocolea minor Nees [Chiloscyphus minor (Nees) J. J. Engel et R. M. Schust.]

Lophozia ascendens (Warnst.) R. M. Schust. [Lophozia gracillima H. Buch]

Lophozia guttulata (Lindb. et Arnell) A. Evans [Lophozia porphyroleuca (Nees) Schiffn. nom. illeg., L. longiflora auct. (sensu GROLLE and LONG 2000; SöDERSTRÖM, URMI, et al. 2002; DAMSHOLT 2002), L. longiflora var. guttulata (Lindb. et Arnell) Schljakov] - The specimens hitherto inserted in L. longiflo$r a$ have been collected from dead wood. They represent $L$. guttulata according to annotation 26 in HodgetTs et al. (2020): 'Since SCHLJA KOV (1980), Lophozia longiflora has been used to include Lophozia guttulata, a species mostly restricted to moist dead wood habitats. Lophozia longiflora was lectotypified by BAKALIN (2016). The lectotype corresponds to the concept of MÜLLER (1954), Saukel (1985), Meinunger and Schröder (2007), Bakalin (2016) and KöCKINGER (2017), describing a species occurring mostly on peaty soil and in rocky habitats, but not the concept of GROLLE and LONG (2000), which corresponds to our Lophozia guttulata.' Characters of stem anatomy as described in MEINUNGER and SCHRÖDER (2007) and of leaf shape and perianth mouth (BAKALIN 2016) were studied in some specimens from the Mátra Mts and confirm this view. Specimens studied: (1) Heves County [8186.1] Mátra Mts, Parád, Northern slope of Mt Kékes, Sötét-Lápa, andesite boulder scree, on decaying log, $c a \mathrm{~N} 47.87639^{\circ}$, E 20.01725 , $790 \mathrm{~m}$ a.s.l., 17.07.1997, leg. P. Erzberger, det. J. Váňa (as L. longiflora (Nees) Schiffn.) (BErzberger 3583,3588 ) soc. Anastrophyllum hellerianum, Nowellia curvifolia; rev. as L. guttulata P. Erzberger 01.07.2020. (2) Heves County [8186.1] Mátra Mts, Mátraháza village, Kékes North Forest Reserve, on decaying log in montane beech forest, $\mathrm{N} 47.874^{\circ}$, E $20.007^{\circ}, 800-900 \mathrm{~m}$ a.s.l., 20.06.1998, leg. P. Ódor, det. J. Váňa (as L. longiflora (Nees) Schiffn.), 09.10.1999, 'Rev.' (conf.) T. Pócs and P. Ódor, 18.10.1999, Herbarium Péter Ódor, Budapest, Hungary, Collection No. 19980620-3 (BP 48629/H). (3) Heves County [8186.1] Mátra Mts, Mátraháza village, Kékes North Forest Reserve, on decaying log in montane beech forest, $\mathrm{N} 47.874^{\circ}$, E 20.007, $800-900 \mathrm{~m}$ a.s.l., 24.09.1999, leg. P. Ódor, det. P. Ódor and T. Pócs (as L. longiflora (Nees) Schiffn.), 17.11.1999, 
Herbarium Péter Ódor, Budapest, Hungary, Collection No. 19990924-2 (BP 47408/H). (4) Borsod-Abaúj-Zemplén County [7989.1] Bükk Mts, in lignis putresc. in sylva Svédfenyves prope Jávorkút, 27.07.1959, leg. (et det.) L. Vajda (as Lophozia porphyroleuca (Nees) Schiffner c. per. cum Nowellia et Dolichotheca silesiaca) (BP 20977/H). (5) Baranya County [9975.1] Mecsek Mts, in valle Egervölgy prope Magyarürög, 27.06.1952, leg. (et det.) L. Vajda (as Lophozia ventricosa (Dicks.) Dumort. var. porphyroleuca (Nees) Hartmann cum Leucobryum glaucum) (BP 5113/H).

Lophozia silvicola H. Buch [Lophozia ventricosa auct. (sensu MÜLlER 1954, SCHLJAKov 1980, MeINUNGer and SCHRöder 2007, KöCKINGER 2017, non Grolle and LONG 2000), Lophozia ventricosa var. silvicola (H. Buch) E. W. Jones] - Plants with biconcentric oil bodies and an overall morphology of L. ventricosa have been collected in Hungary, but have hitherto been included in L. ventricosa. Specimens studied: (1) Heves County [8186.1] Mátra Mts, Parád, northern slope of Mt Kékes, Sötét-Lápa, andesite boulder scree, $\mathrm{N} 47.87628^{\circ}$, E 20.01686 $770 \mathrm{~m}$ a.s.l., 31.03.2017, leg. et det. P. Erzberger (B-Erzberger 22819, 22821, 22823) soc. Trilophozia quinquedentata. (2) Borsod-Abaúj-Zemplén County [7594.3] Zemplén Mts, Nagy-Péter-mennykö at Regéc, andesite rocks, N $48.41969^{\circ}$, E $21.38828^{\circ}, 710 \mathrm{~m}$ a.s.1., 25-26.05.2007, leg. et det. B. Papp (with biconcentric oil bodies) (BP 49318/H). L. silvicola has also been reported from Hungary by BAKALIN (2005) according to SöDERSTRÖM et al. (2007: annotation 234).

Lophozia ventricosa (Dicks.) Dumort. [Lophozia groenlandica auct. (sensu SCHLJAKOV 1980, 1998), L. confertifolia auct. (sensu SCHLJA Kov 1975, 1998, ŞTEFăNUȚ 2008), L. murmanica auct. (sensu SCHLJAKOv 1970), L. ventricosa var. confusa R. M. Schust.] - See annotation 32 in HodgetTs et al. (2020): 'The Lophozia ventricosa/wenzelii complex has never been studied in detail worldwide using both molecular and morphological methods. VILNET et al. (2010) and DE ROO et al. (2007) concentrated on other problems and did not include a sufficient number of specimens of these and related taxa to include the full morphological variation of this group. The taxonomy proposed here is based mostly on morphological studies (which, however, are contradictory!) and is therefore provisional pending future research. The name Lophozia ventricosa has been applied to different concepts since BUCH (1929) described Lophozia silvicola (see also BUCH 1932) and has often included the latter as synonym. The neotype chosen by GROLLE and LONG (2000) for Lophozia ventricosa define it as different from Lophozia silvicola. However, there are some doubts if this neotype represents Lophozia ventricosa sensu SöDERSTRÖM et al. (2016) or is a form of Lophozia wenzelii (see MEINUNGER and SCHRÖDER 2007, KöC KINGER 2017). Lophozia ventricosa is here understood as a plant with the 
overall morphology and anatomy of Lophozia silvicola, but showing homogeneous granular oil bodies. The neotype of GROLLE and LONG (2000) needs a reinvestigation as it approaches what we understand as Lophozia wenzelii.

Lophozia wenzelii (Nees) Steph. [Lophozia groenlandica auct. (sensu DAMSHOLT 1994, 2002, ŞTEFĂNUȚ 2008), L. confertifolia Schiffn. (sensu Damsholt 2002, KöC KINGER 2017), L. ventricosa var. uliginosa Breidl. ex Schiffn., L. iremelensis Schljakov] - See annotation 33 in Hodget ts et al. (2020): Lophozia wenzelii is very close to Lophozia ventricosa according to molecular studies by De Roo et al. (2007) and VILNET et al. (2010), but refer to the annotation under Lophozia ventricosa concerning the neotype of the latter species'.

Lophoziopsis excisa (Dicks.) Konstant. et Vilnet [Lophozia excisa (Dicks.) Dumort.]

Lophoziopsis longidens (Lindb.) Konstant. et Vilnet [Lophozia longidens (Lindb.) Macoun]

Lunularia cruciata (L.) Dumort. ex Lindb.

Mannia fragrans (Balb.) Frye et L. Clark

Mannia triandra (Scop.) Grolle

Marchantia polymorpha L. subsp. polymorpha [Marchantia aquatica (Nees) Burgeff ]

Marchantia polymorpha L. subsp. ruderalis Bischl. et Boissel.-Dub. [Marchantia latifolia Gray]

Marchantia quadrata Scop. [Preissia quadrata (Scop.) Nees]

Marsupella emarginata (Ehrh.) Dumort. [Marsupella emarginata var. pearsonii (Schiffn. ex Macvicar) Jørg.]

Marsupella funckii (F. Weber et D. Mohr) Dumort. [M. ramosa Müll. Frib., Marsupella funckii var. badensis (Schiffn.) Fam.]

Marsupella sprucei (Limpr.) Bernet [Marsupella sprucei var. neglecta (Limpr.) Damsh., Marsupella sprucei var. ustulata (Limpr.) Damsh.]

Mesoptychia badensis (Gottsche ex Rabenh.) L. Söderstr. et Váňa [Lophozia badensis (Gottsche ex Rabenh.) Schiffn., Leiocolea badensis (Gottsche) Jørg., Lophozia badensis var. obtusiloba (Bernet) Schiffn.]

Mesoptychia collaris (Nees) L. Söderstr. et Váňa [Leiocolea collaris (Nees) Schljakov, Lophozia alpestris (Schleich ex F. Weber) Evans nom. rej., Leiocolea alpestris (Schleich. ex F. Weber) Isov., Lophozia alpestris var. libertae (Huebener) Damsh.]

Mesoptychia heterocolpos (Thed. ex Hartm.) L. Söderstr. et Váňa [Lophozia heterocolpos (Thed. ex C. Hartm.) M. Howe, Leiocolea heterocolpos (Thed. ex Hartm.) H. Buch]

Metzgeria conjugata Lindb. [Metzgeria conjugata var. alipila Kaal., Metzgeria conjugata var. macvicarii Kaal.] 
Metzgeria furcata (L.) Corda [Metzgeria furcata var. expansa Douin, Metzgeria furcata var. flexipilis Kaal., Metzgeria furcata var. ulvula (Nees) Pavletic]

Metzgeria pubescens (Schrank) Raddi [Apometzgeria pubescens (Schrank) Kuwah.] Nardia geoscyphus (De Not.) Lindb.

Nardia scalaris Gray

Neoorthocaulis floerkei (F. Weber et D. Mohr) L. Söderstr., De Roo et Hedd. [Barbilophozia floerkei (F. Weber et D. Mohr) Loeske, Orthocaulis floerkei (F. Weber et D. Mohr) H. Buch, Lophozia floerkei (F. Weber et D. Mohr) Schiffn.] Nowellia curvifolia (Dicks.) Mitt.

Obtusifolium obtusum (Lindb.) S. W. Arnell [Lophozia obtusa (Lindb.) A. Evans] Oxymitra incrassata (Brot.) Sérgio et Sim-Sim

Pedinophyllum interruptum (Nees) Kaal.

Pellia epiphylla (L.) Corda

Pellia neesiana (Gottsche) Limpr. - ERZberger (2018).

Plagiochila asplenioides (L.) Dumort. [Plagiochila major (Nees) S. W. Arnell]

Plagiochila porelloides (Torr. ex Nees) Lindenb. [Plagiochila asplenioides subsp. porelloides (Torrey ex Nees) Kaal.]

Porella arboris-vitae (With.) Grolle

Porella baueri (Schiffn.) C. E. O. Jensen

Porella cordaeana (Huebener) Moore [Porella cordaeana var. faeroensis (C. E. O. Jensen) E. W. Jones, Porella cordaeana var. simplicior (J. E. Zetterst.) Arnell] Porella platyphylla (L.) Pfeiff. [Porella platyphylla var. subsquarrosa (Schiffn.) Arnell ]

Ptilidium pulcherrimum (Weber) Vain. [Ptilidium pulcherrimum var. subpinnatum (Jørg.) Damsh.]

Radula complanata (L.) Dumort. [Radula complanata var. alpestris (Lindb. ex Berggr.) Lindb.]

Radula lindenbergiana Gottsche ex C. Hartm. [Radula complanata subsp. lindenbergiana (Gottsche ex C. Hartm.) R. M. Schust., R. lindbergiana Gottsche ex J. B. Jack nom. inval.] - Although the only voucher in BP for this species is sterile and the determination by $\mathrm{R}$. Düll as 'probably $R$. lindenbergiana' must therefore appear doubtful, we accept the species even in the absence of a voucher, based on the literature: Boros (1968) reports a collection with perianths from the Köszeg Mts by Latzel, which therefore was estimated to be reliable, contrary to the sterile collections from other regions. Unfortunately, we could not locate a specimen for the record from the Köszeg Mts.

Reboulia hemisphaerica (L.) Raddi

Riccardia chamedryfolia (With.) Grolle [Riccardia chamedryfolia var. major (Nees) R. M. Schust. nom. illeg., Riccardia chamedryfolia var. submersa (C. E. O. Jensen ex Müll. Frib.) Damsh. nom. inval.] 
Riccardia incurvata Lindb.

Riccardia latifrons (Lindb.) Lindb.

Riccardia multifida (L.) Gray

Riccardia palmata (Hedw.) Carruth.

Riccia bifurca Hoffm. [Riccia bifurca var. subinermis Heeg]

Riccia canaliculata Hoffm. - We follow CASPARI et al. (2018) in including Riccia duplex Lorb. ex Müll. Frib. in R. canaliculata Hoffm.

Riccia cavernosa Hoffm. [Riccia teneriffae S. W. Arnell, Riccia cavernosa var. angustior (Nees) Damsh.]

Riccia ciliata Hoffm. [Riccia ciliata var. epilosa Warnst., $R$. ciliata var. intumescens Bisch., $R$. ciliata var. violacea Kny, $R$. dalslandica S. W. Arnell, $R$. intumescens (Bisch.) Underw., $R$. canescens Steph., $R$. trichocarpa M. Howe, $R$. crinita auct. eur.] - See annotation 150 in HodgetTs et al. (2020): 'Riccia trichocarpa was synonymised with the Australian Riccia crinita (the latter having priority) by JovET-Ast (2000), a synonymisation that has been largely overlooked or rejected by European bryologists. HugONNOT (2010) argued that JovET-AsT's (1986) concept of Riccia trichocarpa is actually Riccia ciliata, and her Riccia ciliata belongs to other species. Thus, Hugonnot synonymised both taxa under the oldest name, Riccia ciliata.' As a consequence, we exclude the name Riccia crinita Taylor from the checklist and use Riccia ciliata Hoffm. instead.

Riccia ciliifera Link [Riccia melitensis C. Massal.]

Riccia crozalsii Levier

Riccia fluitans L.

Riccia frostii Austin

Riccia glauca L. var. glauca [Riccia glauca var. major (Roth) Lindenb.]

Riccia glauca L. var. ciliaris Warnst. [Riccia glauca var. subinermis (Lindb.) Warnst.] - recognised in HodgetTs et al. (2020). Occurrence in Hungary (unpublished): [8079.2] Pest County, Börzsöny, Kemence, Rakottyásbérc, N $47^{\circ} 58^{\prime} 13.1^{\prime \prime}, \mathrm{E} 18^{\circ} 57^{\prime} 08.0^{\prime \prime}$, nyílt andezit sziklagyep, $650 \mathrm{~m}$ a.s.l., 07.08.2016, leg. J. Nagy, det. L. Meinunger (hb. J. Nagy).

Riccia gougetiana Durieu et Mont.

Riccia buebeneriana Lindenb.

Riccia papillosa Moris

Riccia rhenana Lorb. ex Müll. Frib.

Riccia sorocarpa Bisch. subsp. sorocarpa [Riccia sorocarpa var. heegii Schiffn.]

Riccia subbifurca Warnst. ex Croz. [?Riccia oelandica C. E. O. Jensen]

Riccia warnstorfii Limpr. ex Warnst. [Riccia warnstorfii var. commutata (J. B. Jack ex Levier) Damsh., Riccia warnstorfii var. subinermis Warnst., Riccia warnstorfii var. ciliaris Warnst.]

Ricciocarpos natans (L.) Corda 
Scapania aequiloba (Schwägr.) Dumort.

Scapania apiculata Spruce

Scapania aspera M. Bernet et Bernet

Scapania calcicola (Arnell et J. Perss.) Ingham

Scapania curta (Mart.) Dumort.

Scapania irrigua Nees

Scapania lingulata $\mathrm{H}$. Buch

Scapania mucronata $\mathrm{H}$. Buch

Scapania nemorea (L.) Grolle

Scapania parvifolia Warnst. - Already reported in Boros (1968) and OrBÁN and Vajda (1983), but later (Erzberger and PAPp 2004, PAPp et al. 2010) included in S. scandica following Grolle and LONG (2000); now recognised as a species in its own right (HodgetTs et al. 2020). New occurrence in Hungary (unpublished): [7494.4] Zemplén Mts, Borsod-Abaúj-Zemplén County, Füzérkomlós, Tegda-bérc, N 48 31'33.0”, E $21^{\circ} 25^{\prime} 36.1^{\prime \prime}$, on acidic soil between rhyolit rocks, $360 \mathrm{~m}$ a.s.l., 27.08.2015, leg. Németh, det. Németh, conf. Schröder and Erzberger (hb. Cs. Németh 7232).

Scapania praetervisa Meyl. [Scapania mucronata subsp. praetervisa (Meyl.) R. M. Schust.] - ERzBerger and MeINUNGer (2014b).

Scapania scandica (Arnell et H. Buch) Macvicar

Scapania umbrosa (Schrad.) Dumort.

Scapania undulata (L.) Dumort. [Scapania undulata var. aequatiformis (De Not.) C. Massal. et Carestia, Scapania undulata var. dentata (Dumort.) Jørg., Scapania undulata var. oakesii (Austin) H. Buch]

Schistochilopsis incisa (Schrad.) Konstant. [Lophozia incisa (Schrad.) Dumort., Massularia incisa (Schrad.) Schljakov, Schistochilopsis incisa var. inermis (Müll. Frib.) Konstant.]

Solenostoma gracillimum (Sm.) R. M. Schust. [Jungermannia gracillima Sm., Jungermannia gracillima var. crenulata (Mitt.) Damsh.]

Solenostoma byalinum (Lyell) Mitt. [Jungermannia hyalina Lyell, Plectocolea hyalina (Lyell) Mitt.]

Solenostoma sphaerocarpum (Hook.) Steph. [Jungermannia sphaerocarpa Hook., Solenostoma pusillum (C.E. O.Jens.) Steph., Jungermannia jenseniana Grolle, Jungermannia sphaerocarpa var. nana (Nees ex Flot.) Frye et L. Clark nom. illeg., Solenostoma sphaerocarpum var. nanum (Nees ex Flot.) R. M. Schust.] Sphaerocarpos europaeus Lorb. [Sphaerocarpos texanus auct. eur. non Austin] Sphenolobus minutus (Schreb. ex D. Crantz) Berggr. [Anastrophyllum minutum (Schreb.) R. M. Schust., Anastrophyllum minutum var. weberi (Mart.) Kartt.] Syzygiella autumnalis (DC.) K. Feldberg, Váňa, Hentschel et Heinrichs [Jamesoniella autumnalis (DC.) Steph., Crossogyna autumnalis (DC.) Schljakov] 
Trichocolea tomentella (Ehrh.) Dumort.

Trilophozia quinquedentata (Huds.) Bakalin [Tritomaria quinquedentata (Huds.) H. Buch, T. quinquedentata subsp. turgida (Lindb.) Damsh., Trilophozia quinquedentata subsp. turgida (Lindb.) Konstant., Tritomaria quinquedentata $\mathrm{f}$. gracilis (C. E. O. Jensen) R. M. Schust., T. quinquedentata var. dentata S. W. Arnell nom. inval., T. quinquedentata var. grandiretis $\mathrm{H}$. Buch et $\mathrm{S}$. W. Arnell] Tritomaria exsecta (Schmidel) Schiffn. ex Loeske Tritomaria exsectiformis (Breidl.) Schiffn. ex Loeske

\section{Mosses}

Abietinella abietina (Hedw.) M. Fleisch. (Thuidium abietinum (Hedw.) Schimp.) - For Abietinella abietina var. hystricosa (Mitt.) Sakurai see Appendix 3. Acaulon muticum (Hedw.) Müll. Hal. Acaulon triquetrum (Spruce) Müll. Hal. Alleniella besseri (Lobarz.) S. Olsson, Enroth et D. Quandt [Neckera besseri (Lobarz.) Jur.] (Homalia besseri Lobarz.)

Alleniella complanata (Hedw.) S. Olsson, Enroth et D. Quandt [Neckera complanata (Hedw.) Huebener]

Aloina aloides (Koch ex Schultz) Kindb.

Aloina ambigua (Bruch et Schimp.) Limpr.

Aloina rigida (Hedw.) Limpr.

Amblyodon dealbatus (Hedw.) P. Beauv.

Amblystegium serpens (Hedw.) Schimp. [Amblystegium serpens var. salinum Carrington] - Following ERzBerger and PAPP (2004), Amblystegium serpens var. juratzkanum (Schimp.) Rau et Herv. is included; it was treated as a separate taxon in the older Hungarian literature (Boros 1953, 1968, OrBÁN and VAJDA 1983), but it is not recognised in Hodgetts et al. (2020).

Amphidium mougeotii (Schimp.) Schimp.

Anacamptodon splachnoides (Froel. ex Brid.) Brid.

Andreaea rupestris Hedw.

Anomodon longifolius (Schleich. ex Brid.) Hartm. [Anomodontella longifolia (Schleich. ex Brid.) Ignatov et Fedosov]

Anomodon rugelii (Müll. Hal.) Keissl. [Anomodontopsis rugelii (Müll. Hal.) Ignatov et Fedosov]

Anomodon viticulosus (Hedw.) Hook. et Taylor

Antitrichia curtipendula (Hedw.) Brid.

Archidium alternifolium (Hedw.) Mitt.

Atrichum angustatum (Brid.) Bruch et Schimp.

Atrichum flavisetum Mitt. (Atrichum undulatum var. gracilisetum Besch., Atrichum haussknechtii Juratzka et Milde, Catharinea haussknechtii Broth.) - 
recognised as separate species in Boros (1968), ORBÁN and VAJDA (1983), but included in $A$. undulatum in previous checklists (ERZBERGER and PAPP 2004, PAPP et al. 2010).

Atrichum undulatum (Hedw.) P. Beauv.

Aulacomnium androgynum (Hedw.) Schwägr.

Aulacomnium palustre (Hedw.) Schwägr.

Barbula unguiculata Hedw.

Bartramia halleriana Hedw.

Bartramia ithyphylla Brid.

Bartramia pomiformis Hedw.

Blindia acuta (Hedw.) Bruch et Schimp.

Blindiadelphus campylopodus (Kindb.) Fedosov et Ignatov [Seligeria campylopoda Kindb.]

Blindiadelphus recurvatus (Hedw.) Fedosov et Ignatov [Seligeria recurvata (Hedw.) Bruch et Schimp.]

Brachydontium trichodes (F. Weber) Milde [Seligeria transylvanica Plam.]

Brachytheciastrum olympicum (Jur.) Vanderp. et al. - PAPP et al. (2020).

Brachytheciastrum velutinum (Hedw.) Ignatov et Huttunen [Brachytheciastrum vanekii (Šmarda) Ochyra et Żarnowiec, B. velutinum var. vagans (Milde)

Ochyra et Żarnowiec] (Brachythecium velutinum (Hedw.) Schimp.)

Brachythecium albicans (Hedw.) Schimp.

Brachythecium campestre (Müll. Hal.) Schimp.

Brachythecium capillaceum (F. Weber et D. Mohr) Giacom. [Brachythecium rotaeanum De Not.]

Brachythecium gebeebii Milde

Brachythecium glareosum (Bruch ex Spruce) Schimp.

Brachythecium laetum (Brid.) Schimp.

Brachythecium mildeanum (Schimp.) Schimp.

Brachythecium rivulare Schimp.

Brachythecium rutabulum (Hedw.) Schimp.

Brachythecium salebrosum (Hoffm. ex F. Weber et D. Mohr) Schimp.

Brachythecium tommasinii (Sendtn. ex Boulay) Ignatov et Huttunen (Cirriphyllum tommasinii (Boulay) Grout, Brachythecium tenuicaule (Spruce) Kindb., Rhynchostegiella tenuicaulis (Spruce) Kartt., Cirriphyllum germanicum (Grebe) Loeske et M. Fleisch.) - We follow Caspari et al. 2018 and NebeL and Philip PI (2001) and include Brachythecium tenuicaule in the synonymy of $B$. tommasinii. According to Hodgetts et al. (2020, annotation 480), molecular studies by KöCKINGER and KUČERA (2016) also demonstrate a close relationship of Brachythecium tenuicaule to B. tommasinii.

Bruchia flexuosa (Schwägr.) Müll. Hal. - Mesterházy and NÉmeth (2015). 
Bryoerythrophyllum recurvirostrum (Hedw.) P. C. Chen

Bryum argenteum $\mathrm{Hedw}$.

Bryum barnesii J. B. Wood ex Schimp. (Gemmabryum barnesii (J. B. Wood ex Schimp.) J. R. Spence, Osculatia barnesii (J. B. Wood ex Schimp.) Ochyra, Plášek et Bedn.-Ochyra) - Erzberger (2015). Although HolyoAK (2003) included Bryum barnesii in Bryum dichotomum, stating that 'many plants and populations intermediate between Bryum barnesii and Bryum dichotomum occur' (Hodget ts et al. 2020: annotation 363), we follow Central European authors (e.g. CASPARI et al. 2018) in regarding Bryum barnesii as a distinct species and retain the narrower concept of Bryum bicolor Dicks.

Bryum bicolor Dicks. [Osculatia bicolor (Dicks.) Ochyra, Plášek et Bedn.-Ochyra] - see the annotation under Bryum barnesii.

Bryum gemmiferum R. Wilczek et Demaret [Gemmabryum gemmiferum (R. Wilczek et Demaret) J. R. Spence, Osculatia gemmifera (R. Wilczek et Demaret) Ochyra, Plášek et Bedn.-Ochyra]

Bryum gemmilucens $\mathrm{R}$. Wilczek et Demaret [Gemmabryum gemmilucens ( $\mathrm{R}$. Wilczek et Demaret) J. R. Spence, Osculatia gemmilucens (R. Wilczek et Demaret) Ochyra, Plášek et Bedn.-Ochyra]

Bryum klinggraeffii Schimp. [Gemmabryum klinggraeffii (Schimp.) J. R. Spence et H. P. Ramsay, Osculatia klinggraeffii (Schimp.) Ochyra, Plášek et Bedn.Ochyra]

Bryum radiculosum Brid. [Gemmabryum radiculosum (Brid.) J. R. Spence et $\mathrm{H}$. P. Ramsay, Osculatia radiculosa (Brid.) Ochyra, Plášek et Bedn.-Ochyra]

Bryum ruderale Crundw. et Nyholm [Gemmabryum ruderale (Crundw. et Nyholm) J. R. Spence, Osculatia ruderalis (Crundw. et Nyholm) Ochyra, Plášek et Bedn.-Ochyra ]

Bryum stirtonii Schimp. - not recognised in HodgetTs et al. (2020), included in Ptychostomum elegans by many authors, e.g. CASPARI et al. (2018), KöCKINGER et al. (2008). In spite of its controversial taxonomic status, we want to keep this name in the checklist, in order to stimulate further floristical research, see also ERZBERGER and SCHRÖDER (2013).

Bryum violaceum Crundw. et Nyholm [Gemmabryum violaceum (Crundw. et Nyholm) J. R. Spence, Osculatia violacea (Crundw. et Nyholm) Ochyra, Plášek et Bedn.-Ochyra]

Buckia vaucheri (Lesq.) D. Rios, M. T. Gallego et J. Guerra [Hypnum vaucheri Lesq.]

Buxbaumia aphylla Hedw.

Buxbaumia viridis (Moug. ex Lam. et DC.) Brid. ex Moug. et Nestl.

Callicladium haldanianum (Grev.) H. A. Crum - ERZBERGER et al. (2016b).

Calliergon cordifolium (Hedw.) Kindb. 
Calliergon giganteum (Schimp.) Kindb.

Calliergonella cuspidata (Hedw.) Loeske

Calliergonella lindbergii (Mitt.) Hedenäs (Hypnum lindbergii Mitt.)

Campyliadelphus chrysophyllus (Brid.) R. S. Chopra (Campylium chrysophyllum (Brid.) Lange)

Campyliadelphus elodes (Lindb.) Kanda (Campylium elodes (Lindb.) Kindb.)

Campylium protensum (Brid.) Kindb. (Campylium stellatum var. protensum

(Brid.) Bryhn) - See annotation 431 in Hodget ss et al. (2020): 'Campylium protensum and Campylium stellatum are distinct in some parts of their range and overlap in others. They are retained as separate species for the present, although there is a strong case for Campylium protensum to be reduced to a variety of Campylium stellatum, as some authors have done.' Var. protensum is missing in PAPP et al. (2010), but see ERzBerger and PAPP (2004).

Campylium stellatum (Hedw.) Lange et C. E. O. Jensen

Campylophyllopsis calcarea (Crundw. et Nyholm) Ochyra [Campylidium calcareum (Crundw. et Nyholm) Ochyra, Campylophyllum calcareum (Crundw. et Nyholm) Hedenäs]

Campylopus flexuosus (Hedw.) Brid. - ERzBerger and Németh (2014).

Campylopus fragilis (Brid.) Bruch et Schimp. - Deme et al. (2015).

Campylopus introflexus (Hedw.) Brid.

Campylopus pyriformis (Schultz) Brid. [Campylopus pyriformis var. azoricus (Mitt.) M. F. V. Corley]

Campylostelium saxicola (F. Weber et D. Mohr) Bruch et Schimp.

Ceratodon purpureus (Hedw.) Brid.

Chenia leptophylla (Müll. Hal.) R. H. Zander [Leptophascum leptophyllum (Müll. Hal.) J. Guerra et M. J. Cano] (Phascum leptophyllum Müll. Hal.)

Chionoloma tenuirostre (Hook. et Taylor) M. Alonso, M. J. Cano et J. A. Jiménez [Oxystegus tenuirostris (Hook. et Taylor) A. J. E. Sm., Trichostomum tenuirostre (Hook. et Taylor) Lindb.]

Cinclidotus danubicus Schiffn. et Baumgartner

Cinclidotus fontinaloides (Hedw.) P. Beauv.

Cinclidotus riparius (Host ex Brid.) Arn. [Cinclidotus confertus Lüth]

Cirriphyllum crassinervium (Taylor) Loeske et M. Fleisch. (Eurhynchium crassinervium (Wilson) Schimp.)

Cirriphyllum piliferum (Hedw.) Grout

Claopodium rostratum (Hedw.) Ignatov [Anomodon rostratus (Hedw.) Schimp.]

Climacium dendroides (Hedw.) F. Weber et D. Mohr

Cnestrum schisti (F. Weber et D. Mohr) I. Hagen - Among several collections

inserted in BP under this name, only a single specimen could be confirmed (Papp, unpublished): Comit. Heves [8185.2] (Mátra Mts) In rupibus ande- 
sit. silvat. sept. montis Saskő prope Parád, 8-890 m, 27.04.1942, leg. et det. Boros, Á. (BP 97612).

Codonoblepharon forsteri (Dicks.) Goffinet [Zygodon forsteri (Dicks.) Mitt.] PAPP and Sinigla (2017).

Conardia compacta (Drumm. ex Müll. Hal.) H. Rob.

Coscinodon cribrosus (Hedw.) Spruce [Grimmia cribrosa Hedw.]

Cratoneuron filicinum (Hedw.) Spruce

Crossidium crassinervium (De Not.) Jur. - spelled 'crassinerve' in ERZBERGER and PAPP (2004) and PAPP et al. (2010).

Crossidium laxefilamentosum W. Frey et Kürschner - According to HodgetTs et al. (2020: annotation 241), Crossidium laxefilamentosum is doubtfully separable from Crossidium crassinervium.

Crossidium squamiferum (Viv.) Jur. var. squamiferum - NAGY and ERZBERGER (2018).

Ctenidium molluscum (Hedw.) Mitt.

Cynodontium polycarpon (Hedw.) Schimp. - spelled 'polycarpum' in ERZBERGER and PAPP (2004) and PAPP et al. (2010).

Cynodontium strumiferum (Hedw.) Lindb. (Cynodontium polycarpon var. strumiferum (Hedw.) Schimp.) - var. strumiferum is missing in PAPP et al. (2010), but see ERzBERGER and PAPP (2004).

Cynodontium tenellum (Schimp.) Limpr.

Dichodontium pellucidum (Hedw.) Schimp.

Dicranella cerviculata (Hedw.) Schimp.

Dicranella crispa (Hedw.) Schimp.

Dicranella beteromalla (Hedw.) Schimp.

Dicranella bowei Renauld et Cardot

Dicranella bumilis $\mathrm{R}$. Ruthe

Dicranella rufescens (Dicks.) Schimp.

Dicranella schreberiana (Hedw.) Dixon

Dicranella staphylina $\mathrm{H}$. Whitehouse

Dicranella subulata (Hedw.) Schimp.

Dicranella varia (Hedw.) Schimp.

Dicranodontium denudatum (Brid.) E. Britton [Dicranodontium denudatum var. alpinum (Schimp.) I. Hagen]

Dicranoweisia cirrata (Hedw.) Lindb.

Dicranum bonjeanii De Not. [Dicranum undulatum Turner, nom. illeg.]

Dicranum flagellare Hedw. [Orthodicranum flagellare (Hedw.) Loeske]

Dicranum fulvum Hook. [Orthodicranum fulvum (Hook.) G. Roth ex CasaresGil]

Dicranum montanum Hedw. [Orthodicranum montanum (Hedw.) Loeske] 
Dicranum muehlenbeckii Bruch et Schimp.

Dicranum polysetum Sw. ex anon. [Dicranum rugosum (Hoffm. ex Funck) Brid., Dicranum undulatum Ehrh. ex F. Weber et D. Mohr, nom. illeg.]

Dicranum scoparium Hedw.

Dicranum spurium Hedw.

Dicranum tauricum Sapjegin [Dicranum strictum Schleich. ex D. Mohr, hom. illeg., Orthodicranum tauricum (Sapjegin) Smirnova]

Dicranum viride (Sull. et Lesq.) Lindb.

Didymodon acutus (Brid.) K. Saito

Didymodon cordatus Jur. [Vinealobryum cordatum (Jur.) R. H. Zander]

Didymodon fallax (Hedw.) R. H. Zander [Geheebia fallax (Hedw.) R. H. Zander] Didymodon ferrugineus (Schimp. ex Besch.) M. O. Hill [Geheebia ferruginea (Schimp. ex Besch.) R. H. Zander]

Didymodon glaucus Ryan

Didymodon insulanus (De Not.) M. O. Hill [Vinealobryum insulanum (De Not.) R. H. Zander]

Didymodon luridus Hornsch. [Vinealobryum luridum (Hornsch.) R. H. Zander] Didymodon rigidulus $\mathrm{Hedw}$.

Didymodon sinuosus (Mitt.) Delogne [Vinealobryum sinuosum (Mitt.) R. H. Zander]

Didymodon spadiceus (Mitt.) Limpr. [Geheebia spadicea (Mitt.) R. H. Zander] Didymodon tophaceus (Brid.) Lisa subsp. erosus (J. A. Jiménez et J. Guerra) Jan Kučera [Didymodon erosus J. A. Jiménez et J. Guerra] - KuČERA et al. (2018). Didymodon tophaceus (Brid.) Lisa subsp. sicculus (M. J. Cano, Ros, García-Zam.

et J. Guerra) Jan Kučera [Didymodon sicculus M. J. Cano, Ros, García-Zam. et J. Guerra] - KUČERA et al. (2018).

Didymodon tophaceus (Brid.) Lisa subsp. tophaceus

Didymodon vinealis (Brid.) R. H. Zander [Vinealobryum vineale (Brid.) R. H. Zander]

Diphyscium foliosum (Hedw.) D. Mohr

Distichium capillaceum (Hedw.) Bruch et Schimp.

Ditrichum beteromallum (Hedw.) E. Britton

Ditrichum lineare (Sw.) Lindb. - NÉMETH (2016).

Ditrichum pallidum (Hedw.) Hampe

Ditrichum pusillum (Hedw.) Hampe

Drepanocladus aduncus (Hedw.) Warnst.

Drepanocladus lycopodioides (Brid.) Warnst. [Pseudocalliergon lycopodioides (Brid.) Hedenäs]

Drepanocladus polygamus (Schimp.) Hedenäs (Campylium polygamum (Schimp.) C. E. O.Jensen) - See annotation 438 in Hodget Ts et al. (2020): 'Campylium 
decipiens (Warnst.) Walsemann is a central European plant recognised as a distinct species by FraHM and WALSEMANN (1973) and MEINUNGER and SCHRÖDER (2007), but otherwise largely disregarded. It may be a form of Drepanocladus polygamus, but it requires further study.' See also ERZBERGER and NÉmet t (2015) and the note under Campylium decipiens in Appendix 3.

Drepanocladus sendtneri (Schimp. ex H. Müll.) Warnst.

Drepanocladus sordidus (Müll. Hal.) Hedenäs

Encalypta ciliata Hedw.

Encalypta rhaptocarpa Schwägr. - ERZBERGER (2014).

Encalypta streptocarpa Hedw.

Encalypta vulgaris $\mathrm{Hedw}$.

Entodon concinnus (De Not.) Paris

Entosthodon fascicularis (Hedw.) Müll. Hal.

Entosthodon hungaricus (Boros) Loeske

Entosthodon mublenbergii (Turner) Fife (Funaria mublenbergii Turner) - misspelled 'mueblenbergii' in PAPP et al. (2010).

Entosthodon pulchellus (H. Philib.) Brugués (Funaria pulchella H. Philib.)

Ephemerum cohaerens (Hedw.) Hampe

Ephemerum crassinervium (Schwägr.) Hampe subsp. sessile (Bruch) Holyoak [Ephemerum sessile (Bruch) Müll. Hal.]

Ephemerum recurvifolium (Dicks.) Boulay

Ephemerum serratum (Hedw.) Hampe [Ephemerum minutissimum Lindb., $E$. serratum var. minutissimum (Lindb.) Grout] - 'As a consequence of lectotypification, Ephermerum minutissimum Lindb. was placed in synonymy with Ephemerum serratum (Hedw.) Hampe (Ellis and PRICE 2015)' (HODGETTS et al. 2020: annotation 283). We would like to discourage the use of the name $E$. serratum without any clarification, because it is ambiguous: sensu Ellis and PRICE (2015) it stands for what was formerly named $E$. minutissimum, but in long established use it formerly meant $E$. stoloniferum.

Ephemerum stoloniferum (Hedw.) L. T. Ellis et M. J. Price [Ephemerum serratum auct., Ephemerum stellatum H. Philib.] - 'Ephemerum stellatum was synonymised with Ephemerum serratum by HOLYOAK (2010). Subsequently, ELLIS and PRICE (2015) lectotypified E. stoloniferum and showed that it was the correct name for the large-spored taxon previously treated under the name E. serratum' (HodGETTS et al. 2020: annotation 284).

Eucladium verticillatum (With.) Bruch et Schimp. [Eucladium verticillatum var. angustifolium Lindb]

Eurbynchiastrum pulchellum (Hedw.) Ignatov et Huttunen [Eurhynchiastrum pulchellum var. praecox (Hedw.) Ochyra et Żarnowiec] (Eurhynchium pulchellum (Hedw.) Jenn.) 
Eurhynchium angustirete (Broth.) T. J. Kop.

Eurbynchium striatum (Hedw.) Schimp.

Exsertotheca crispa (Hedw.) S. Olsson, Enroth et D. Quandt [Neckera crispa Hedw.]

Fabronia ciliaris (Brid.) Brid.

Fabronia pusilla Raddi

Fissidens adianthoides Hedw.

Fissidens arnoldii R. Ruthe

Fissidens bryoides Hedw.

Fissidens crassipes Wilson ex Bruch et Schimp. - Contrary to Hodgetts et al. (2020), we follow BRUGUÉs and GUERRA (2015) and include subsp. warnstorfii (M. Fleisch.) Brugg.-Nann. in the synonymy of $F$. crassipes. See also ERZBERGER (2016b).

Fissidens crispus Mont. [Fissidens herzogii R. Ruthe ex Herzog, Fissidens limbatus Sull., Fissidens minutulus Sull.] - ERZBERGER (2016a,b).

Fissidens curvatus Hornsch. [Fissidens algarvicus Solms]

Fissidens dubius P. Beauv. var. dubius [Fissidens cristatus Wilson ex Mitt.]

Fissidens dubius P. Beauv. var. mucronatus (Limpr.) Kartt., Hedenäs et L. Söderstr. - ERzBERger (2016b), see also HodgetTs et al. (2020: annotation 213) 'Fissidens dubius var. mucronatus is morphologically characterised by the mucronate apex of the upper leaves. It also has a different ecology. Recent Dutch research also shows differences in DNA sequences between var. mucronatus and the type (H. N. Siebel and M. Stech, pers. comm. 2019)'.

Fissidens exilis Hedw.

Fissidens gracilifolius Brugg.-Nann. et Nyholm [Fissidens viridulus var. tenuifolius (Boulay) A. J. E. Sm.]

Fissidens gymnandrus Buse

Fissidens incurvus Starke ex Röhl. [Fissidens viridulus var. incurvus (Starke ex Röhl.) Waldh.]

Fissidens pusillus (Wilson) Milde [Fissidens viridulus var. pusillus Wilson]

Fissidens taxifolius Hedw. [Fissidens taxifolius subsp.pallidicaulis (Mitt.) Mönk.]

Fissidens viridulus (Sw.) Wahlenb. [Fissidens bambergeri Milde, Fissidens exiguus

Sull., Fissidens viridulus var. bambergeri (Schimp.) Waldh.] - see the notes under F. bambergeri and F. exiguus in Appendix 3.

Flexitrichum flexicaule (Schwägr.) Ignatov et Fedosov [Ditrichum flexicaule (Schwägr.) Hampe]

Flexitrichum gracile (Mitt.) Ignatov et Fedosov [Ditrichum crispatissimum (Müll. Hal.) Paris, Ditrichum gracile (Mitt.) Kuntze]

Fontinalis antipyretica Hedw.

Fontinalis hypnoides $\mathrm{C}$. Hartm.

Funaria hygrometrica Hedw. 
Grimmia anodon Bruch et Schimp.

Grimmia crinita Brid.

Grimmia decipiens (Schultz) Lindb.

Grimmia dissimulata E. Maier

Grimmia elatior Bruch ex Bals.-Criv. et De Not.

Grimmia funalis (Schwägr.) Bruch et Schimp.

Grimmia hartmanii Schimp.

Grimmia laevigata (Brid.) Brid. [Guembelia laevigata (Brid.) Ochyra et Żarnowiec ]

Grimmia lisae De Not.

Grimmia longirostris Hook. [Guembelia longirostris(Hook.) Ochyra et Żarnowiec]

Grimmia montana Bruch et Schimp. [Orthogrimmia montana (Bruch et Schimp.) Ochyra et Żarnowiec]

Grimmia mueblenbeckii Schimp.

Grimmia orbicularis Bruch ex Wilson [Dryptodon orbicularis (Bruch ex Wilson) Ochyra et $\dot{Z}$ arnowiec]

Grimmia ovalis (Hedw.) Lindb. [Guembelia ovalis (Hedw.) Müll. Hal.]

Grimmia plagiopodia Hedw.

Grimmia pulvinata (Hedw.) Sm.

Grimmia teretinervis Limpr.

Grimmia tergestina Tomm. ex Bruch et Schimp. [Grimmia tergestina var. tergestinoides (Culm.) Podp., Guembelia tergestina (Tomm. ex Bruch et Schimp.) Buyss.] - 'Grimmia tergestina var. tergestinoides seems distinct in Central Europe. More work is needed to determine its taxonomic value' (annotation 328 in HodgetTs et al. 2020). Because of this taxonomic uncertainty, we refrain from recognising var. tergestinoides, in spite of the fact that a specimen of var. tergestinoides from the Bükk Mts is cited in ERzBERGER and PAPP (2004).

Grimmia trichophylla Grev. [Grimmia britannica A. J. E. Sm.]

Gymnostomum aeruginosum Sm.

Gymnostomum calcareum Nees et Hornsch. [Gymnostomum calcareum var. lanceolatum Sérgio, Gymnostomum lanceolatum M. J. Cano, Ros et J. Guerra ]

Gymnostomum viridulum Brid.

Gyroweisia tenuis (Hedw.) Schimp.

Hamatocaulis vernicosus (Mitt.) Hedenäs

Hedwigia ciliata (Hedw.) P. Beauv.

Hedwigia emodica Hampe ex Müll. Hal. [Hedwigia ciliata var. leucophaea Bruch et Schimp.] - 'HEDENäs (1994) suggested that Hedwigia emodica was a synonym of Hedwigia ciliata var. leucophaea. BUCHBENDER et al. (2014) later proved the hybrid origin of some accessions referable to this taxon. IGNATOVA et al. 
(2016) recognised the taxon at species level, as Hedwigia emodica, but doubts persist that all the European material is the same as the plants from Russia (or the Himalayan type of Hedwigia emodica)' (Hodgetts et al. 2020: annotation 346). In Hungary, $H$. ciliata var. leucophaea occurs in two morphs: One with straight, appressed leaves in the dry state, which corresponds to the photographs published in IGNATOVA et al. (2016), and another one with dry leaves squarrosely reflexed, in habit not different from $H$. stellata (Erzberger, unpublished observations). Preliminary molecular work kindly undertaken by J. Kučera on Hungarian specimens (unpublished) unfortunately did not yield unequivocal results, not least due to insufficient sampling of the Russian and Asian material examined in IgNATOva et al. (2016).

Hedwigia stellata Hedenäs

Helodium blandowii (F. Weber et D. Mohr) Warnst. [Elodium blandowii (F. Weber et D. Mohr) Eckel]

Henediella heimii (Hedw.) R. H. Zander [Desmatodon wilczekii Meyl., Tortula rhodonia R. H. Zander] (Desmatodon heimii (Hedw.) Mitt.)

Herzogiella seligeri (Brid.) Z. Iwats.

Heterocladiella dimorpha (Brid.) Ignatov et Fedosov [Heterocladium dimorphum (Brid.) Schimp.]

Heterocladium heteropterum (Brid.) Schimp. - BARÁTH and ERZBERGER (2017a). Hilpertia velenovskyi (Schiffn.) R. H. Zander

Homalia trichomanoides (Hedw.) Brid.

Homalothecium lutescens (Hedw.) H. Rob.

Homalothecium philippeanum (Spruce) Schimp.

Homalothecium sericeum (Hedw.) Schimp.

Homomallium incurvatum (Schrad. ex Brid.) Loeske

Hookeria lucens (Hedw.) Sm. - Ódor and SzURDOKI (2011).

Hydrogonium consanguineum (Thwaites et Mitt.) Hilp. [Barbula consanguinea (Thwaites et Mitt.) A. Jaeger] (Barbula indica (Hook.) Spreng.) - 'Hydrogonium consanguineum was earlier reported from Europe as Barbula indica; KöcKINGER et al. (2012) proved that these reports belong to Barbula consanguinea, transferred to Hydrogonium by KUČERA et al. (2013)' (HODGETTS et al. 2020: annotation 291).

Hygroamblystegium fluviatile (Hedw.) Loeske (Amblystegium fluviatile (Hedw.) Schimp.)

Hygroamblystegium humile (P. Beauv.) Vanderp., Goffinet et Hedenäs [Hygroamblystegium varium var. humile Vanderp. et Hedenäs] (Amblystegium bumile (P. Beauv.) Crundw.

Hygroamblystegium tenax (Hedw.) Jenn. (Amblystegium tenax (Hedw.) C. E. O. Jensen) 
Hygroamblystegium varium (Hedw.) Mönk. [Amblystegium varium (Hedw.) Lindb.] Hygrobypnum luridum (Hedw.) Jenn.

Hylocomiadelphus triquetrus (Hedw.) Ochyra et Stebel [Rhytidiadelphus triquetrus (Hedw.) Warnst.]

Hylocomium splendens (Hedw.) Schimp.

Hymenostylium recurvirostrum (Hedw.) Dixon [Gymnostomum recurvirostrum Hedw.]

Hypnum cupressiforme Hedw. var. cupressiforme

Hypnum cupressiforme Hedw. var. lacunosum Brid.

Hypnum cupressiforme Hedw. var. subjulaceum Molendo [Hypnum subcomplanatum Hedenäs, Schlesak et D. Quandt, nom. illeg., H. subjulaceum (Molendo) Hedenäs, Schlesak et D. Quandt] (Hypnum cupressiforme var. cuspidatum Jur.) - Hypnum subjulaceum was published by SCHLESAK et al. (2018) as H. subcomplanatum rather than $H$. subjulaceum because the latter was thought to be blocked by the name $H$. subjulaceum Besch. However, the latter name was not validly published, so its use at species level is not blocked. This was corrected by SCHLESAK et al. (2019). Later, KuČERA et al. (2019) found that Hypnum subjulaceum formed a supported lineage within $H$. cupressiforme s. lat., and so it was returned to varietal status (HoDGETTS et al. 2020, annotation 492). The variety is reported by Boros (1968) as H. cupressiforme var. cuspidatum Jur. for the Bükk Mts and Mátra Mts, but its occurrence in Hungary was considered doubtful by Düll (in litt., see ERZBERGER and PAPP 2004). Inter alia, the following specimen from the Börzsöny Mts was confirmed: Herbarium Musei Hist. Nat. Hung. Budapest. Flora Hungarica. Comit Nógrád. In rupibus siccis montis Oltárkő prope pag. Diósjenő, montes Börzsöny, 02.10.1957, leg. et det. L. Vajda (BP 58014) conf. B. Papp, 25.06.2020 [8079.4, ca $800 \mathrm{~m}$ a.s.l.].

Hypnum jutlandicum Holmen et E. Warncke

Hypnum pallescens (Hedw.) P. Beauv. var. reptile (Michx.) Husn. - HodgetTs et al. (2020) include Hypnum pallescens var. reptile in a broadly understood taxon Jochenia pallescens (Hedw.) Hedenäs, Schlesak et D. Quandt, in the family Jocheniaceae Jan Kučera et Ignatov. However, in accordance with CASPARI et al. (2018) and based on the experience of MEINUNGER and SCHRÖDER (2007), who separated Hypnum reptile at species level from $H$. pallescens and described in detail their morphological, ecological and chorological differences, we here retain the name $H$. pallescens var. reptile for the Hungarian plants, which are clearly different from var. pallescens as found, e.g. in the Alps. As a consequence, we cannot recognise the family Jocheniaceae for the bryoflora of Hungary.

Imbribryum alpinum (Huds. ex With.) N. Pedersen [Bryum alpinum Huds. ex With.]

Imbribryum mildeanum (Jur.) J. R. Spence [Bryum mildeanum Jur.] 
Imbribryum subapiculatum (Hampe) D. Bell et Holyoak [Bryum subapiculatum Hampe, Gemmabryum subapiculatum (Hampe) J. R. Spence et H. P. Ramsay, Osculatia subapiculata (Hampe) Ochyra, Plášek et Bedn.-Ochyra] - SCHRÖDER and ERZBERGER (2012).

Imbribryum tenuisetum (Limpr.) D. Bell et Holyoak [Bryum tenuisetum Limpr., Gemmabryum tenuisetum (Limpr.) J. R. Spence et H. P. Ramsay, Osculatia tenuiseta (Limpr.) Ochyra, Plášek et Bedn.-Ochyra] - Erzberger and SCHRÖDER (2016).

Isopterygiopsis pulchella (Hedw.) Z. Iwats. - The status of this species in Hungary is controversial. Köckinger (2016, pers. comm.) doubted the occurrence of $I$. pulchella in Hungary, and some representative specimens were revised to Orthothecium intricatum (Hartm.) Schimp. by Hedenäs (2016, unpublished). Interestingly, some of the old collections by Boros in BP were originally identified as $O$. intricatum. More work is needed to decide on the status of I. pulchella in Hungary.

Isothecium alopecuroides (Lam. ex Dubois) Isov.

Isothecium myosuroides Brid. [Isothecium myosuroides subsp. brevinerve Kindb.]

Kindbergia praelonga (Hedw.) Ochyra

Leptobryum pyriforme (Hedw.) Wilson

Leptodictyum riparium (Hedw.) Warnst.

Leptodon smithii (Hedw.) F. Weber et D. Mohr

Lescuraea saviana (De Not.) E. Lawton [Pseudoleskea saviana (De Not.) Latzel]

Leskea polycarpa $\mathrm{Hedw}$.

Leucobryum glaucum (Hedw.) Ångstr.

Leucobryum juniperoideum (Brid.) Müll. Hal.

Leucodon sciuroides (Hedw.) Schwägr. [Leucodon sciuroides var. morensis (Schwägr.) De Not.]

Lewinskya affinis (Schrad. ex Brid.) F. Lara, Garilleti et Goffinet [Orthotrichum affine Schrad. ex Brid., O. affine var. bohemicum Plášek et Sawicki]

Lewinskya rupestris (Schleich. ex Schwägr.) F. Lara, Garilleti et Goffinet [Orthotrichum rupestre Schleich. ex Schwägr.]

Lewinskya speciosa (Nees) F. Lara, Garilleti et Goffinet [Orthotrichum speciosum Nees]

Lewinskya striata (Hedw.) F. Lara, Garilleti et Goffinet [Orthotrichum striatum Hedw.] - Lewinskya shawii (Wilson) F. Lara, Garilleti et Goffinet (Orthotrichum shawii Wilson), which was mentioned in ERzBERGER and PAPP (2004) based on a report in Boros (1968), does not occur in Hungary (specimens revised by Kirmaci, Papp and Erzberger, unpublished).

Loeskeobryum brevirostre (Brid.) M. Fleisch. (Hylocomium brevirostre (Brid.) Schimp.) 
Meesia triquetra (L. ex Jolycl.) Ångstr.

Microbryum curvicollum (Hedw.) R. H. Zander (Phascum curvicolle Hedw.)

Microbryum davallianum (Sm.) R. H. Zander (Pottia davalliana (Sm.) C. E. O. Jensen)

Microbryum floerkeanum (F. Weber et D. Mohr) Schimp. (Phascum floerkeanum F. Weber et D. Mohr)

Microbryum muticum (Venturi) Cl. Schneid., Th. Schneid. et Mahévas (Pottia mutica Venturi) - This taxon is not recognised in Hodgetts et al. (2020). We follow CASPARI $e t$ al. (2018) for the combination under Microbryum.

Microbryum starckeanum (Hedw.) R. H. Zander (Pottia starckeana (Hedw.) Müll. Hal.)

Microeurbynchium pumilum (Wilson) Ignatov et Vanderp. [Oxyrrhynchium pumilum (Wilson) Loeske] (Eurhynchium pumilum (Wilson) Schimp.)

Mnium bornum Hedw.

Mnium lycopodioides Schwägr.

Mnium marginatum (Dicks.) P. Beauv.

Mnium spinulosum Bruch et Schimp.

Mnium stellare Hedw.

Mnium thomsonii Schimp.

Myurella julacea (Schwägr.) Schimp.

Neckera pennata Hedw.

Neckera pumila Hedw.

Nogopterium gracile (Hedw.) Crosby et W. R. Buck [Pterogonium gracile (Hedw.) Sm. nom. illeg.]

Nybolmiella gymnostoma (Bruch ex Brid.) Holmen et E. Warncke [Orthotrichum gymnostomum Bruch ex Brid.]

Nybolmiella obtusifolia (Brid.) Holmen et E. Warncke [Orthotrichum obtusifolium Brid.]

Orthodontium lineare Schwägr. - SzÜcs and BIDLó (2012).

Orthothecium intricatum (Hartm.) Schimp.

Orthotrichum anomalum Hedw.

Orthotrichum cupulatum Brid. - HodgetTs et al. (2020) distinguish several varieties, but in Hungary only the typical variety occurs, contrary to reports of var. riparium in Boros (1968), also mentioned in ERZBERGER and PAPP (2004); the corresponding specimens represent var. cupulatum and a mixture of $O$. cupulatum var. cupulatum and $O$. anomalum, respectively (Papp, unpublished revision).

Orthotrichum diaphanum Brid.

Orthotrichum pallens Bruch ex Brid.

Orthotrichum patens Bruch ex Brid. 
Orthotrichum pumilum Sw. ex anon.

Orthotrichum rogeri Brid.

Orthotrichum schimperi Hammar - O. schimperi was treated by Boros (1968) as a variety of $O$. pumilum, and thus included in the latter in PAPP et al. (2010). However, recently the taxon has been elevated to species rank, and we therefore follow HoDGETTS et al. (2020) in considering it a species in its own right. The species has many recent records.

Orthotrichum sprucei Mont.

Orthotrichum stramineum Hornsch. ex Brid.

Orthotrichum urnigerum Myrin

Oxyrrbynchium hians (Hedw.) Loeske (Eurhynchium hians (Hedw.) Sande Lac.) Oxyrrhynchium schleicheri (R. Hedw.) Röll (Eurhynchium schleicheri (R. Hedw.) Jur.)

Oxyrrhynchium speciosum (Brid.) Warnst. (Eurhynchium speciosum (Brid.) Jur.) Palustriella commutata (Hedw.) Ochyra

Palustriella falcata (Brid.) Hedenäs - PAPP et al. (2020).

Paraleucobryum longifolium (Hedw.) Loeske

Philonotis caespitosa Jur.

Philonotis calcarea (Bruch et Schimp.) Schimp.

Philonotis capillaris Lindb. [Philonotis arnellii Husn.] - Philonotis capillaris is the correct name for Philonotis arnellii (Koponen and Isovirta 2010, HoDGETTS et al. 2020: annotation 353).

Philonotis fontana (Hedw.) Brid.

Philonotis marchica (Hedw.) Brid.

Physcomitrium eurystomum Sendtn.

Physcomitrium patens (Hedw.) Mitt. [Aphanorrhegma patens (Hedw.) Lindb., Physcomitrella patens (Hedw.) Bruch et Schimp.]

Physcomitrium pyriforme (Hedw.) Bruch et Schimp.

Physcomitrium sphaericum (C. F. Ludw. ex Schkuhr) Brid.

Plagiomnium affine (Blandow ex Funck) T. J. Kop. [Orthomnion affine (Blandow ex Funck) T. J. Kop. et Yu Sun]

Plagiomnium cuspidatum (Hedw.) T. J. Kop. [Orthomnion cuspidatum (Hedw.) T. J. Kop. et Yu Sun]

Plagiomnium elatum (Bruch et Schimp.) T. J. Kop. [Orthomnion elatum (Bruch et Schimp.) T. J. Kop. et Yu Sun]

Plagiomnium ellipticum (Brid.) T. J. Kop. [Orthomnion ellipticum (Brid.) T. J. Kop. et Yu Sun]

Plagiomnium medium (Bruch et Schimp.) T. J. Kop. [Orthomnion medium (Bruch et Schimp.) T. J. Kop. et Yu Sun] 
Plagiomnium rostratum (Schrad.) T. J. Kop. [Orthomnion rostratum (Schrad.) T. J. Kop. et Yu Sun]

Plagiomnium undulatum (Hedw.) T. J. Kop. [Orthomnion undulatum (Hedw.) T. J. Kop. et Yu Sun]

Plagiopus oederianus (Sw.) H. A. Crum et L. E. Anderson

Plagiothecium cavifolium (Brid.) Z. Iwats.

Plagiothecium curvifolium Schlieph. ex Limpr.

Plagiothecium denticulatum (Hedw.) Schimp. var. denticulatum

Plagiothecium denticulatum (Hedw.) Schimp. var. undulatum R. Ruthe ex Geh. (Plagiothecium ruthei Limpr.) - This taxon has been reduced from specific rank to variety in HodGETTs et al. (2020).

Plagiothecium laetum Schimp.

Plagiothecium latebricola Schimp. - BARÁTH and ERZBERger (2017b).

Plagiothecium nemorale (Mitt.) A. Jaeger

Plagiothecium platyphyllum Mönk.

Plagiothecium succulentum (Wilson) Lindb.

Plagiothecium undulatum (Hedw.) Schimp. [Buckiella undulata (Hedw.) Ireland] Plasteurbynchium striatulum (Spruce) M. Fleisch. (Eurbynchium striatulum (Spruce) Schimp.)

Platydictya jungermannioides (Brid.) H. A. Crum

Platygyrium repens (Brid.) Schimp.

Pleuridium acuminatum Lindb.

Pleuridium subulatum (Hedw.) Rabenh.

Pleurozium schreberi (Willd. ex Brid.) Mitt.

Pogonatum aloides (Hedw.) P. Beauv.

Pogonatum nanum (Hedw.) P. Beauv.

Pogonatum urnigerum (Hedw.) P. Beauv.

Poblia andalusica (Höhn.) Broth.

Poblia annotina (Hedw.) Lindb.

Pohlia camptotrachela (Renauld et Cardot) Broth.

Poblia cruda (Hedw.) Lindb.

Poblia elongata $\mathrm{Hedw}$.

Poblia lescuriana (Sull.) Ochi

Poblia lutescens (Limpr.) H. Lindb.

Poblia melanodon (Brid.) A. J. Shaw

Poblia nutans (Hedw.) Lindb. subsp. nutans

Pohlia nutans (Hedw.) Lindb. subsp. schimperi (Müll. Hal.) Nyholm (Pohlia schimperi (Müll. Hal.) A. L. Andrews) - This taxon has been reduced from specific rank to that of subspecies in HodGETTS et al. (2020).

Pohlia proligera (Kindb.) Lindb. ex Broth. 
Poblia wahlenbergii (F. Weber et D. Mohr) A. L. Andrews

Polytrichastrum alpinum (Hedw.) G. L. Sm. [Polytrichastrum norwegicum (Hedw.)

Schljakov, Polytrichum alpinum Hedw., P. alpinum var. arcticum (Sw. ex

Brid.) Wahlenb.]

Polytrichum commune Hedw. [Polytrichum commune var. commune, P. commune

var. uliginosum Wallr., P. uliginosum (Wallr.) Schriebl]

Polytrichum formosum Hedw. [Polytrichastrum formosum (Hedw.) G. L. Sm.]

Polytrichum juniperinum Hedw.

Polytrichum longisetum Sw. ex Brid. [Polytrichastrum longisetum (Sw. ex Brid.) G. L. Sm.]

Polytrichum perigoniale Michx. [Polytrichum commune var. bumile Sw., P. commune var. perigoniale (Michx.) Hampe] - var. perigoniale is missing in PAPP et al. (2010), but see ERZBERgER and PAPP (2004).

Polytrichum piliferum Hedw.

Polytrichum strictum Menzies ex Brid. [Polytrichum alpestre Hoppe]

Pseudanomodon attenuatus (Hedw.) Ignatov et Fedosov [Anomodon attenuatus (Hedw.) Huebener]

Pseudephemerum nitidum (Hedw.) Loeske

Pseudoamblystegium subtile (Hedw.) Vanderp. et Hedenäs [Amblystegium subtile (Hedw.) Schimp.]

Pseudocampylium radicale (P. Beauv.) Vanderp. et Hedenäs [Amblystegium radicale (P. Beauv.) Schimp.]

Pseudocrossidium hornschuchianum (Schultz) R. H. Zander

Pseudocrossidium revolutum (Brid.) R. H. Zander

Pseudoleskeella catenulata (Brid. ex Schrad.) Kindb.

Pseudoleskeella nervosa (Brid.) Nyholm

Pseudoscleropodium purum (Hedw.) M. Fleisch. (Scleropodium purum (Hedw.) Limpr.)

Pseudotaxiphyllum elegans (Brid.) Z. Iwats.

Pterigynandrum filiforme Hedw. [Pterigynandrum filiforme var. majus (De Not.) De Not.]

Pterygoneurum lamellatum (Lindb.) Jur.

Pterygoneurum ovatum (Hedw.) Dixon [Pterygoneurum compactum M. J. Cano, J. Guerra et Ros, Pterygoneurum crossidioides W. Frey, Herrnst. et Kürschner, Pterygoneurum squamosum Segarra et Kürschner] - 'Synonymies of Pterygoneurum ovatum follow GUERRA et al. 2006. However, this treatment is not yet supported by molecular data' (HoDGETTs et al. 2020: annotation 259). In consequence, we exclude $P$. compactum, $P$. crossidioides and $P$. squamosum from the updated Hungarian checklist.

Pterygoneurum subsessile (Brid.) Jur. 
Ptilium crista-castrensis (Hedw.) De Not.

Ptychostomum capillare (Hedw.) Holyoak et N. Pedersen [Rosulabryum capillare (Hedw.) J. R. Spence, Bryum capillare Hedw.]

Ptychostomum cernuum (Hedw.) Hornsch. [Bryum uliginosum (Brid.) Bruch et Schimp.]

Ptychostomum compactum Hornsch. [Bryum algovicum Sendtn. ex Müll. Hal., Bryum compactum (Hornsch.) Kindb.]

Ptychostomum creberrimum (Taylor) J. R. Spence et H. P. Ramsay [Bryum creberrimum Taylor]

Ptychostomum elegans (Nees) D. Bell et Holyoak [Bryum elegans Nees, Rosulabryum elegans (Nees) Ochyra]

Ptychostomum funkii (Schwägr.) J. R. Spence [Bryum funkii Schwägr.] - For the spelling 'funkii' see annotation 379 in HodgetTs et al. (2020).

Ptychostomum imbricatulum (Müll. Hal.) Holyoak et N. Pedersen [Bryum caespiticium Hedw., Gemmabryum caespiticium (Hedw.) J. R. Spence, Osculatia caespiticia (Hedw.) Ochyra, Plášek et Bedn.-Ochyra]

Ptychostomum inclinatum (Sw. ex Brid.) J. R. Spence [Bryum archangelicum Bruch et Schimp., Ptychostomum archangelicum (Bruch et Schimp.) J. R. Spence] (Bryum imbricatum (Schwägr.) Bruch et Schimp.) - For the nomenclatural issues see annotation 380 in HodgetTs et al. (2020).

Ptychostomum intermedium (Brid.) J. R. Spence [Bryum intermedium (Brid.) Blandow]

Ptychostomum moravicum (Podp.) Ros et Mazimpaka [Bryum moravicum Podp., Rosulabryum laevifilum (Syed) Ochyra]

Ptychostomum pallens (Sw. ex anon.) J. R. Spence [Bryum pallens Sw. ex anon., Bryum sibiricum Lindb. et Arnell]

Ptychostomum pallescens (Schleich. ex Schwägr.) J. R. Spence [Bryum pallescens Schleich. ex Schwägr., Ptychostomum boreale (F. Weber et D. Mohr) Ochyra et Bedn.-Ochyra] - we include Ptychostomum lonchocaulon (Müll. Hal.) J. R. Spence (Bryum lonchocaulon Müll. Hal.) in a broadly conceived P.pallescens. See also Appendix 3.

Ptychostomum pseudotriquetrum (Hedw.) J. R. Spence et H. P. Ramsay ex Holyoak et N. Pedersen var. pseudotriquetrum [Bryum neodamense Itzigs., Bryum pseudotriquetrum (Hedw.) P. Gaertn., B. Mey. et Scherb., Ptychostomum neodamense (Itzigs.) J. R. Spence] - 'Ptychostomum neodamense has been shown by HOLYOAK and HEDENÄs (2006), from molecular and other data, to represent an inconstant phenotype of Ptychostomum pseudotriquetrum occurring locally in highly calcareous habitats prone to inundation, and connected to it by intermediate forms known widely in Europe and also in Asia, Alaska and Greenland. Its retention at species rank by SPENCE (2014) is a 
consequence of an over-emphasis on morphological data' (HODGETTs et al. 2020: annotation 386). In consequence, we exclude Ptychostomum (Bryum) neodamense from the updated Hungarian checklist.

Ptychostomum pseudotriquetrum (Hedw.) J. R. Spence et H. P. Ramsay ex Holyoak et N. Pedersen var. bimum (Schreb.) Holyoak et N. Pedersen [Bryum pseudotriquetrum var. bimum (Schreb.) Lilj.] - var. bimum is missing in PAPP et al. (2010), but see Erzberger and Papp (2004).

Ptychostomum rubens (Mitt.) Holyoak et N. Pedersen [Bryum rubens Mitt., Osculatia rubens (Mitt.) Ochyra, Plášek et Bedn.-Ochyra, Rosulabryum rubens (Mitt.) J. R. Spence]

Ptychostomum torquescens (Bruch et Schimp.) Ros et Mazimpaka [Bryum torquescens Bruch et Schimp., Rosulabryum torquescens (Bruch et Schimp.) J. R. Spence]

Ptychostomum turbinatum (Hedw.) J. R. Spence [Bryum turbinatum (Hedw.) Turner]

Ptychostomum warneum (Röhl.) J. R. Spence [Bryum warneum (Röhl.) Brid.]

Ptychostomum weigelii (Biehler) J. R. Spence [Bryum weigelii Biehler]

Ptychostomum zieri (Hedw.) Holyoak et N. Pedersen [Plagiobryum zieri (Hedw.) Lindb.] - spelled as 'zierii' in ERzBERger and PAPP (2004) and PAPP et al. (2010).

Pulvigera lyellii (Hook. et Taylor) Plášek, Sawicki et Ochyra [Orthotrichum lyellii Hook. et Taylor]

Pylaisia polyantha (Hedw.) Schimp.

Pyramidula tetragona (Brid.) Brid.

Racomitrium aciculare (Hedw.) Brid. [Codriophorus acicularis (Hedw.) P. Beauv.]

Racomitrium affine (F. Weber et D. Mohr) Lindb. [Bucklandiella affinis (Schleich. ex F. Weber et D. Mohr) Bedn.-Ochyra et Ochyra]

Racomitrium aquaticum (Brid. ex Schrad.) Brid. [Codriophorus aquaticus (Brid. ex Schrad.) Bedn.-Ochyra et Ochyra]

Racomitrium canescens (Hedw.) Brid. [Niphotrichum canescens (Hedw.) Bedn.Ochyra et Ochyra]

Racomitrium heterostichum (Hedw.) Brid. [Bucklandiella heterosticha (Hedw.) Bedn.-Ochyra et Ochyra]

Racomitrium lanuginosum (Hedw.) Brid. - According to PAPP et al. (2010) the presence of this species in Hungary was based on a single herbarium specimen (Cottus Castriferrei, S-Kápolna (Sorkikápolna) in silvis, without year, leg. J. Márton BP 6630). However, later doubts arose concerning the trustworthiness of the collector, József Márton (1860-1895), whose herbarium also holds other taxa that did not grow in the reported localities (A. Mesterházy, pers. comm.). But even if this doubtful record is excluded, the presence 
of $R$. lanuginosum in Hungary is out of question, since, fortunately, a new occurrence has recently been discovered: [8182.2] Nógrád County, Cserhát, Bér, Nagy-hegy, Kötenger, on andesite boulders, N $47^{\circ} 51^{\prime} 56.91^{\prime \prime}, \mathrm{E} 19^{\circ} 28^{\prime}$ 41.38”, 365 m a.s.l., 18.07.2020, leg. Csiky János and Csikyné Radnai Éva, det. Csiky János, conf. P. Erzberger, J. Csiky, pers. comm.

Racomitrium microcarpon (Hedw.) Brid. [Bucklandiella microcarpa (Hedw.) Bedn.-Ochyra et Ochyra] - BednAREK-Ochyra et al. (2011).

Rhabdoweisia crispata (Dicks.) Lindb. - MESTERHÁzy et al. (2017).

Rhabdoweisia fugax (Hedw.) Bruch et Schimp.

Rhizomnium punctatum (Hedw.) T. J. Kop.

Rhodobryum ontariense (Kindb.) Kindb.

Rhodobryum roseum (Hedw.) Limpr.

Rhynchostegiella curviseta (Brid.) Limpr.

Rhynchostegiella tenella (Dicks.) Limpr.

Rhynchostegiella teneriffae (Mont.) Dirkse et Bouman [Rhynchostegiella jacquinii (Garov.) Limpr., Rhynchostegiella macilenta (Renauld et Cardot) Cardot, Rhynchostegiella teesdalei (Schimp.) Limpr.]

Rhynchostegium confertum (Dicks.) Schimp.

Rhynchostegium megapolitanum (Blandow ex F. Weber et D. Mohr) Schimp.

Rhynchostegium murale (Hedw.) Schimp. [Rhynchostegium arcticum (I. Hagen)

Ignatov et Huttunen]

Rhynchostegium riparioides (Hedw.) Cardot [Platyhypnidium grolleanum Ochyra et Bedn.-Ochyra, Platyhypnidium torrenticola (Ochyra, C. Schmidt et Bültmann) Ochyra et Bedn.-Ochyra] (Platyhypnidium riparioides (Hedw.) Dixon) Rhynchostegium rotundifolium (Scop. ex Brid.) Schimp.

Rhytidiadelphus squarrosus (Hedw.) Warnst.

Rhytidium rugosum (Hedw.) Kindb.

Saelania glaucescens (Hedw.) Broth.

Sanionia uncinata (Hedw.) Loeske

Sarmentypnum exannulatum (Schimp.) Hedenäs [Warnstorfia exannulata (Schimp.) Loeske]

Schistidium apocarpum (Hedw.) Bruch et Schimp.

Schistidium brunnescens Limpr. subsp. brunnescens

Schistidium brunnescens Limpr. subsp. griseum (Nees et Hornsch.) H. H. Blom Schistidium confertum (Funck) Bruch et Schimp.

Schistidium confusum $\mathrm{H}$. H. Blom

Schistidium crassipilum $\mathrm{H}$. H. Blom

Schistidium dupretii (Thér.) W. A. Weber

Schistidium elegantulum $\mathrm{H}$. H. Blom subsp. elegantulum

Schistidium flaccidum (De Not.) Ochyra 
Schistidium helveticum (Schkuhr) Deguchi [Schistidium singarense (Schiffn.) Laz.] Schistidium lancifolium (Kindb.) H. H. Blom

Schistidium papillosum Culm.

Schistidium platyphyllum (Mitt.) H. Perss.

Schistidium pruinosum (Wilson ex Schimp.) G. Roth

Schistidium robustum (Nees et Hornsch.) H. H. Blom

Sciuro-bypnum curtum (Lindb.) Ignatov - EC KSTEIN et al. (2017), see also annotation 487 in HodgetTs et al. (2020): 'Sciuro-hypnum curtum was restored from synonymy with Sciuro-hypnum oedipodium by IgNATOV and MiLYUTINA (2007). It is a widespread species in Europe, whereas Sciuro-hypnum oedipodium, which is primarily a western North American species, is very rare, with just a few records from Eastern Europe'.

Sciuro-bypnum flotowianum (Sendtn.) Ignatov et Huttunen (Eurhynchium flotowianum (Sendtn.) Kartt.)

Sciuro-bypnum plumosum (Hedw.) Ignatov et Huttunen (Brachythecium plumosum (Hedw.) Schimp.)

Sciuro-hypnum populeum (Hedw.) Ignatov et Huttunen (Brachythecium populeum (Hedw.) Schimp.)

Sciuro-hypnum reflexum (Starke) Ignatov et Huttunen (Brachythecium reflexum (Starke) Schimp.)

Scorpidium cossonii (Schimp.) Hedenäs (Drepanocladus cossonii (Schimp.) Loeske) Scorpidium scorpioides (Hedw.) Limpr.

Seligeria acutifolia Lindb. - NÉMETH et al. (2016).

Seligeria calcarea (Hedw.) Bruch et Schimp.

Seligeria donniana (Sm.) Müll. Hal. [Seligeria galinae Mogensen et I. Goldberg] Seligeria patula (Lindb.) I. Hagen [Seligeria alpestris T. Schauer, S. patula var. alpestris (T. Schauer) Gos et Ochyra, S. tristichoides var. patula (Lindb.) Broth.] - Occurrence in Hungary is based on an unpublished revision by $\mathrm{L}$. Gos, which R. Ochyra (pers. comm.) kindly brought to our attention. Specimen: Herbar. Musei Hist. Nat. Hung. Budapest, Flora Hungarica, Comit. Veszprém. In rupibus calcareis umbrosis vallis Ördögárok, prope Gézaháza, montes Bakony [8673.4] 5. VII. 1969, leg. et det. L. Vajda (as Seligeria calcarea (Dicks.) Br. eur.) (BP 74406). Seligeria tristichoides Kindb. var. patula (Lindb.) Broth. Revised by Lidia Gos 1991.

Seligeria pusilla (Hedw.) Bruch et Schimp.

Seligeria trifaria (Brid.) Lindb. var. longifolia (Lindb. ex Broth.) Ochyra et Gos Sematophyllum adnatum (Michx.) E. Britton - ERZBERGER et al. (2018b).

Serpoleskea confervoides (Brid.) Schimp. [Amblystegium confervoides (Brid.) Schimp.]

Sphagnum angustifolium (C. E. O. Jensen ex Russow) C. E. O. Jensen 
Sphagnum auriculatum Schimp. [Sphagnum denticulatum Brid.]

Sphagnum capillifolium (Ehrh.) Hedw. [Sphagnum capillifolium subsp. capillifolium]

Sphagnum centrale C. E. O. Jensen [Sphagnum palustre var. centrale (C. E. O. Jensen) A. Eddy]

Sphagnum compactum Lam. et DC.

Sphagnum contortum Schultz

Sphagnum cuspidatum Ehrh. ex Hoffm.

Sphagnum divinum Flatberg et Hassel [Sphagnum magellanicum auct. eur. p. p., non Brid.] - 'All European records of Sphagnum magellanicum are referable to Sphagnum divinum or Sphagnum medium (HASSEL et al.2018). Sphagnum magellanicum s. str. is confined to southern South America' (Hodget al. 2020: annotation 164). Accoding to E. Szurdoki (pers. comm.), all Hungarian specimens inserted under 'Sphagnum magellanicum' in BP and EGR represent Sphagnum divinum.

Sphagnum fallax (H. Klinggr.) H. Klinggr.

Sphagnum fimbriatum Wilson

Sphagnum flexuosum Dozy et Molk.

Sphagnum girgensobnii Russow

Sphagnum inundatum Russow

Sphagnum obtusum Warnst.

Sphagnum palustre L.

Sphagnum platyphyllum (Lindb. ex Braithw.) Warnst.

Sphagnum quinquefarium (Braithw.) Warnst.

Sphagnum riparium Ångstr.

Sphagnum russowii Warnst.

Sphagnum squarrosum Crome

Sphagnum subnitens Russow et Warnst.

Sphagnum subsecundum Nees

Sphagnum teres (Schimp.) Ångstr.

Sphagnum warnstorfii Russow

Splachnobryum obtusum (Brid.) Müll. Hal.

Straminergon stramineum (Dicks. ex Brid.) Hedenäs (Calliergon stramineum (Brid.) Kindb.)

Streblotrichum convolutum (Hedw.) P. Beauv. var. commutatum (Jur.) J. J. Amann [Barbula convoluta var. sardoa Schimp., Streblotrichum commutatum (Jur.) Hilp.] - var. commutata is not listed in PAPP et al. (2010), but has been mentioned in ERzBerger and PAPP (2004), although as being controversial. Meanwhile, the occurrence of the variety in Hungary has been confirmed from 20 floristical grid cells during recording (Erzberger et al., unpublished). 
Streblotrichum convolutum (Hedw.) P. Beauv. var. convolutum [Barbula convoluta Hedw.]

Syntrichia calcicola J. J. Amann (Tortula calcicolens W. A. Kramer)

Syntrichia caninervis Mitt. var. gypsophila (J. J. Amann ex G. Roth) Ochyra (Tortula caninervis (Mitt.) Broth. subsp. spuria (J. J. Amann) W. A. Kramer)

Syntrichia laevipila Brid. [Syntrichia pagorum (Milde) J. J. Amann] (Tortula laevipila (Brid.) Schwaegr.)

Syntrichia latifolia (Bruch ex Hartm.) Huebener (Tortula latifolia Bruch ex Hartm.)

Syntrichia montana Nees var. calva (Durieu et Sagot ex Bruch et Schimp.) J. J. Amann (Tortula crinita var. calva (Durieu et Sagot) Nebel et Heinrichs) var. calva is missing in PAPP et al. (2010), but see ERzBERGER and PAPP (2004).

Syntrichia montana Nees var. montana [Syntrichia intermedia Brid.] (Tortula crinita (De Not.) De Not.)

Syntrichia norvegica F. Weber (Tortula norvegica (F. Weber) Wahlenb. ex Lindb.) Syntrichia papillosa (Wilson) Jur. (Tortula papillosa Wilson)

Syntrichia ruraliformis (Besch.) Mans. [Syntrichia ruralis var. ruraliformis (Besch.) Delogne] (Tortula ruraliformis (Besch.) Ingham)

Syntrichia ruralis (Hedw.) F. Weber et D. Mohr var. epilosa (Venturi) J. J. Amann - 'Syntrichia ruralis var. epilosa was resurrected by GALLEGo et al. (2018). It may represent a special phenotype within Syntrichia ruraliformis or Syntrichia ruralis, but more study is required (HEDENÄs et al. 2019)' (HoDGETTS et al. 2020: annotation 264). Occurrence in Hungary: ERzBERGER et al. (2018a).

Syntrichia ruralis (Hedw.) F. Weber et D. Mohr var. ruralis [Syntrichia densa (Velen.) J.-P. Frahm, Syntrichia glabra J.-P. Frahm et M. T. Gallego] (Tortula ruralis (Hedw.) P. Gaertn., B. Mey. et Scherb.)

Syntrichia subpapillosissima (Bizot et R. B. Pierrot ex W. A. Kramer) M. T. Gallego et J. Guerra (Tortula papillosissima (Copp.) Broth. var. submamillosa (W. A. Kramer) Heinrichs et Caspari) - var. submamillosa is not listed in PAPP et al. (2010), but see ERzBERGER and PAPP (2004). Contrary to TótH (1986), the typical variety (var. papillosissima $=$ Syntrichia papillosissima (Copp.) Loeske) does not occur in Hungary (U. Abts, unpublished). See also annotation 266 in HodgetTs et al. (2020): 'Syntrichia subpapillosissima may represent a special phenotype within Syntrichia ruraliformis or Syntrichia ruralis, but more study is required (HEDENÄs et al. 2019)'. The species has many recent records.

Syntrichia virescens (De Not.) Ochyra (Tortula virescens (De Not.) De Not.) Taxiphyllum densifolium (Lindb. ex Broth.) Reimers 
Taxiphyllum wissgrillii (Garov.) Wijk et Margad.

Tetraphis pellucida Hedw.

Thamnobryum alopecurum (Hedw.) Gangulee

Thamnobryum neckeroides (Hook.) E. Lawton - Occurrence in Hungary is based on an unpublished revision by M. Mastracci. Specimen: Herbar. Musei Nat. Hungar. Budapest, Flora Hungarica Comit. Nógrád. In rupibus umbrosis supra vall. rivi Rakottyáspatak prope Királyháza, montes Börzsöny [8079.2] 09.05.1959, leg. et det. L. Vajda (as Thamnium alopecurum (L.) Br. eur. fo. pratensa Turn.) with a handwritten note (in Á. Boros' writing) reading: 'Isothecium myurum nem lehet a teljesen más sejthálózat miatt. Keskeny, hosszú levél sejtjei vannak. Climacium-nak széles, háromszögalakú levelei vannak. Sejthálózata a Thamnium-éhez hasonló, középere is fogas, de levélformája más és nagyon concav, míg a Th.-é lapos, ovális, élesen hegyes' (transl.: It cannot be Isothecium myurum because of its totally different areolation. It has narrow, long leaf cells. Climacium has broad triangular leaves. The areolation is similar to that of Thamnium, also the midrib is denticulate, but the leaf shape is different, very concave, whereas Th. has flat, ovate, sharply acute leaves.) (BP 62151) Thamnobryum neckeroides (Hook.) E. Lawton, rev. M. Mastracci 2003.

Thuidium assimile (Mitt.) A. Jaeger (Thuidium philibertii Limpr.)

Thuidium delicatulum (Hedw.) Schimp.

Thuidium recognitum (Hedw.) Lindb.

Thuidium tamariscinum (Hedw.) Schimp.

Timmia austriaca Hedw.

Timmia bavarica Hessl.

Tomentypnum nitens (Hedw.) Loeske

Tortella fasciculata (Culm.) Culm. [Tortella bambergeri auct., non (Schimp.) Broth. p. p., Tortella tortuosa subsp. fasciculata Culm.] - 'Tortella fasciculata was resurrected by KöCKINGER and HEDENÄs (2017) as part of their revision of Tortella bambergeri' (HODGETTS et al. 2020: annotation 299). A Hungarian collection by Boros was studied by KöcKINGER and HedenÄs (2017). See also ERzBERGER and PAPP (2018).

Tortella inclinata (R. Hedw.) Limpr.

Tortella pseudofragilis (Thér.) Köckinger et Hedenäs [Tortella bambergeri auct., non (Schimp.) Broth. p. p., Tortella fragilis var. moravica Podp.] - 'The name Tortella pseudofragilis was introduced by KöcKINGER and HEDENÄs (2017) as part of their revision of Tortella bambergeri' (HODGETTs et al. 2020: annotation 303). Occurrence in Hungary: CASPARI and ERzBERgER (2019), see also ERZBERGER and PAPP (2018).

Tortella squarrosa (Brid.) Limpr. [Pleurochaete squarrosa (Brid.) Lindb.] 
Tortella tortuosa (Hedw.) Limpr. [Tortella bambergeri (Schimp.) Broth.] - 'Using morphological and molecular methods, KöcKINGER and HEDENÄs (2017) demonstrated that the type of Tortella bambergeri was synonymous with Tortella tortuosa. Plants recently treated as Tortella bambergeri are referable to Tortella fasciculata and Tortella pseudofragilis. Up to now, the acceptance of Tortella tortuosa var. fragilifolia (Jur.) Limpr. has been based on the treatment of ECKEL (1998), but this concept is based on plants later referred to Tortella bambergeri (ECKEL 2010). In general, the name 'var. fragilifolia' has been used for morphs of Tortella tortuosa with fragile leaves, and the variety is therefore not included in the checklist' (HodgETTs et al. 2020: annotation 306).

Tortula acaulon (With.) R. H.Zander var. acaulon [Phascum cuspidatum Hedw.] Tortula acaulon (With.) R. H. Zander var. papillosa (Lindb.) R. H. Zander [Phascum cuspidatum var. papillosum (Lindb.) G. Roth] - Phascum cuspidatum var. papillosum Limpr. is missing in PAPP et al. (2010), but mentioned in ERZberger and PAPp (2004). Meanwhile its occurrence in Hungary has been established in 19 floristical grid cells during recording (Erzberger et al., unpublished). Another variety, var. mitraeforme Limpr., also mentioned in ERZBERgER and PAPP (2004), has been preliminarily synonymised with var. papillosum, based on available descriptions, but original material should also be checked (CASPARI et al. 2018).

Tortula acaulon (With.) R. H. Zander var. pilifera (Hedw.) R. H. Zander [Phascum cuspidatum var. piliferum (Hedw.) Hook. et Taylor] - Phascum cuspidatum var. piliferum (Hedw.) Hook. et Taylor is missing in PAPP et al. (2010), but mentioned in ERZBERGER and PAPP (2004) and was already reported by Boros (1968). Meanwhile its occurrence in Hungary has been established in 51 floristical grid cells during recording (Erzberger et al., unpublished).

Tortula atrovirens $(\mathrm{Sm}$.) Lindb.

Tortula brevissima Schiffn.

Tortula caucasica Broth. [Pottia intermedia (Turner) Fürnr., Tortula modica R. $\mathrm{H}$. Zander]

Tortula cernua (Huebener) Lindb. (Desmatodon cernuus (Huebener) Bruch et Schimp.)

Tortula inermis (Brid.) Mont.

Tortula lindbergii Broth. [Pottia lanceolata (Hedw.) Müll. Hal., Tortula lanceola R. H. Zander]

Tortula mucronifolia Schwägr.

Tortula muralis Hedw. subsp. muralis var. aestiva Hedw. - included in Tortula muralis in PAPP et al. (2010), but mentioned in ERZBERGER and PAPP (2004). 'The two varieties of Tortula muralis subsp. muralis intergrade, but 
there is a certain level of distinctness, so this treatment follows KošNAR and KOLÁ ́̌ (2009)' (HodGETTS et al. 2020: annotation 270).

Tortula muralis Hedw. subsp. muralis var. muralis

Tortula muralis Hedw. subsp. obtusifolia (Schwägr.) Culm. [Tortula obtusifolia (Schwägr.) Mathieu] - 'KošNAR and KolÁř (2009) reduced Tortula obtusifolia to a subspecies of Tortula muralis' (HODGETTs et al. 2020: annotation 271).

Tortula protobryoides R. H. Zander [Protobryum bryoides (Dicks.) J. Guerra et M. J. Cano] (Pottia bryoides (Dicks.) Mitt.)

Tortula schimperi M. J. Cano, O. Werner et J. Guerra [Tortula subulata var. angustata (Schimp.) Limpr.]

Tortula subulata Hedw. [Tortula subulata var. graeffi $i$ Warnst., Tortula subulata var. subinermis (Bruch et Schimp.) Wilson]

Tortula truncata (Hedw.) Mitt. (Pottia truncata (Hedw.) Bruch et Schimp.)

Trichodon cylindricus (Hedw.) Schimp. [Ditrichum cylindricum (Hedw.) Grout]

Trichostomum brachydontium Bruch

Trichostomum crispulum Bruch

Ulota bruchii Hornsch. ex Brid.

Ulota coarctata (P. Beauv.) Hammar

Ulota crispa (Hedw.) Brid. - 'The treatment of the Ulota crispa complex (Ulota crispa s. str., Ulota crispula, Ulota intermedia) follows CAPARRós et al. (2016)' (HodgetTs et al. 2020: annotation 418).

Ulota crispula Bruch [Ulota crispa var. crispula (Bruch) Hammar] - The presence of Ulota crispula in Hungary was first demonstrated by CAPARRós et al. (2016). Meanwhile there are data from 77 floristical grid cells (Erzberger et al., unpublished).

Ulota hutchinsiae (Sm.) Hammar

Ulota intermedia Schimp. [Ulota crispa var. intermedia (Schimp.) Cardot] - The presence of Ulota intermedia in Hungary was first demonstrated by CAPARRós et al. (2016). Meanwhile there are data from 19 floristical grid cells (Erzberger $e t$ al., unpublished).

Weissia brachycarpa (Nees et Hornsch.) Jur.

Weissia condensa (Voit) Lindb.

Weissia controversa $\mathrm{Hedw}$. var. controversa

Weissia controversa Hedw. var. crispata (Nees et Hornsch.) Nyholm (Weissia fallax Sehlm.)

Weissia longifolia Mitt.

Weissia rostellata (Brid.) Lindb.

Weissia rutilans (Hedw.) Lindb.

Zygodon rupestris Schimp. ex Lorentz 


\section{DISCUSSION}

Compared to the latest checklist (PAPP et al. 2010), 59 additional taxa (Appendix 1) have been added to the list, but not all of these represent 'true' additions to the bryoflora of Hungary, since taxonomic re-evaluation also contributes to these additional names. True additions to the Hungarian bryoflora (Appendix 1.1) since 2010 comprise 4 species and 2 infraspecific taxa (varieties) of liverworts, and 29 species and 5 infraspecific taxa ( 2 subspecies and 3 varieties) of mosses, altogether 40 taxa. Due to taxonomic changes, another 19 taxa that were known to be part of the Hungarian bryoflora but were not recognised in the previous checklist have been added (Appendix 1.2), 4 species of liverworts and 8 species and 7 varieties of mosses.

On the other hand, 29 taxa have been excluded from the bryophyte flora of Hungary, 7 liverworts and 22 mosses (Appendix 2).

As a result, we obtain a total number of 689 taxa in the Hungarian bryoflora (Table 1).

Table 1. Number of taxa in different Hungarian checklists.

\begin{tabular}{lcccc}
\hline & Hornworts & Liverworts & Mosses & total \\
\hline ERzBERgER and PAPP (2004) & 2 & 143 & 484 & 629 \\
PAPP et al. (2010) & 2 & 146 & 511 & 659 \\
'true' additions (Appendix 1.1) & 0 & 6 & 34 & 40 \\
'taxonomic additions' (Appendix 1.2) & 0 & 4 & 15 & 19 \\
exclusions (Appendix 2) & 0 & 7 & 22 & 29 \\
result present checklist & 2 & 149 & 538 & 689 \\
\hline
\end{tabular}

For some critical or otherwise problematic taxa we feel that our decision not to incorporate them into the checklist of the Hungarian bryoflora is preliminary, these taxa were reported from Hungary but for various reasons cannot be verified satisfactorily. They are compiled and annotated in Appendix 3.

Finally, in Appendix 4 an abridged version of the checklist (without the taxa of Appendices 1 and 2) is arranged according to traditional nomenclature in order to facilitate comparison with previous publications and as a table of synonyms, which seems necessary since there are more than 150 names unfamiliar to bryological non-experts.

Checklists usually are out of date the moment they are printed, yet we hope that much of our work can serve as a taxonomic and nomenclatural basis for a future red list. 
Acknowledgements - Thanks are due to U. Abts, J. Csiky, L. Hedenäs, N. Hodgetts, Th. Kiebacher, M. Kirmaci, G. Kis, H. Köckinger, D. Kovács, J. Kučera, M. Mastracci, L. Meinunger†, J. Nagy, Cs. Németh, R. Ochyra, Chr. Schröck, W. Schröder† and E. Szurdoki for various contributions of unpublished results, examination of critical specimens and discussions on taxonomic and other issues. We would also like to thank all unnamed contributors to the recording project.

Összefoglaló: Az alábbi „checklist” alapján a magyar mohaflóra 2 becősmoha fajból ( 2 nemzetség, 2 család), 146 májmoha fajból és 3 faj alatti taxonból ( 1 alfaj és 2 változat), amelyek 60 nemzetségbe és 34 családba tartoznak, valamint 521 lombosmoha fajból és 17 faj alatti taxonból ( 5 alfaj és 12 változat) áll, amelyek 186 nemzetségbe és 64 családba tartoznak. Így a magyar mohaflóra összesen 669 fajt és további 6 alfajt és 14 változatot, azaz 689 taxont számlál. Az eltelt 10 év alatt 40 mohataxont (6 májmohát és 34 lombosmohát) először mutattak ki a magyar mohaflórában (1.1. Függelék) és további 19 taxon (4 májmoha és 15 lombosmoha), amelyek már korábban a magyar mohaflóra tagjai voltak, de az előző „checklist”-ben nem szerepeltek, most ismét felkerültek a listára (1.2. Függelék). Más részről, 29 taxont (7 májmohát és 22 lombosmohát) jelen ismereteink alapján ki kell zárni a magyar mohaflórából (2. Függelék). A 3. Függelékben további 13 taxont (2 májmohát és 11 lombosmohát) találunk, amelyeket jelenleg nem tekintünk a magyar mohaflóra tagjainak, egy kivételével nem szerepeltek az előző „checklist”-ben, de említésre kerültek valamely más irodalomban és öt kivételével szerepelnek az európai „checklist”-ben. Ezekről a taxonokról nem rendelkezünk még elég információval, hogy megfelelően dönthessünk róluk. A fent említett függelékekben az egyes fajokról bővebb magyarázatokat is adunk, hogy helyzetük megfelelö értékelését segítsük. A 4. Függelékben az összes fajnevet feltüntetjük, amelyek az előző „checklist”-ben szerepeltek, és hozzájuk rendeljük aktuálisan elfogadott nevüket.

\section{REFERENCES}

Bakalin, V. A. (2005): Monograficheskaia obrabotka roda Lophozia (Dumort.) Dumort. s. str. [Monograph of the genus Lophozia (Dumort.) Dumort. s. str.]. - Nauka, Moscow.

BAKalin, V. A. (2016): Notes on Lophozia VIII. The Lectotypification of Lophozia longiflora (Nees) Schiffn. (Lophoziaceae, Hepaticae). - Herzogia 29: 635-643.

https://doi.org/10.13158/heia.29.2.2016.635

BARÁth, K. and ERZberger, P. (2017a): 16. Heterocladium heteropterum (Brid.) Schimp. - In: Ellis, L. T. (ed.): New national and regional bryophyte records, 50. - J. Bryol. 39: 99-114. https://doi.org/10.1080/03736687.2016.1259931

Baráth, K. and Erzberger, P. (2017b): 21. Plagiothecium latebricola Schimp. - In: Ellis, L. T. (ed.): New national and regional bryophyte records, 52. - J. Bryol. 39: 285-394. https://doi.org/10.1080/03736687.2017.1341752

Bartha, D., Király, G., Schmidt, D. and Tiborcz, V. (eds) (2015): Magyarország edényes növényfajainak elterjedési atlasza. (Distribution atlas of vascular plants of Hungary). University of West Hungary Press, Sopron.

Bednarek-Ochyra, H., Erzberger, P. and Ochyra, R. (2011): 5. Bucklandiella microcarpa (Hedw.) Bednarek-Ochyra \& Ochyra. - In: Ellis, L. T. (ed.): New national and regional bryophyte records, 29. - J. Bryol. 33: 316-323.

https://doi.org/10.1179/1743282011Y.0000000031

Boros, Á. (1953): Magyarország mohái. Bryophyta Hungariae. - Akadémiai Kiadó Budapest, 360 pp. Boros, Á. (1968): Bryogeographie und Bryoflora Ungarns. - Akadémiai Kiadó, Budapest, 466 pp. 
Brugués, M. and Guerra, J. (eds) (2015): Flora Briofítica Ibérica. Vol. II, Archidiales: Archidiaceae, Dicranales: Bruchiaceae, Ditrichaceae, Rhabdoweisiaceae, Dicranaceae, Leucobryaceae, Fissidentales: Fissidentaceae, Seligeriales: Seligeriaceae, Grimmiales: Grimmiaceae, Ptychomitriaceae. Universidad de Murcia, Sociedad Española de Briología, Murcia.

Buch, H. (1929): Eine neue moossystematische Methodik. - In: Winge, Ø. (ed.): Beretning om, det 18. Skandinaviske naturforskermøde i København 26-31. August 1929. Frederiksberg Bogtrykkeri, København, pp. 225-229.

Buch, H. (1932): Vorarbeiten zu einer Lebermoosflora Fenno-scandias. I. Ein Versuch zur Aufspaltung der Gattungen Lophozia Dum. und Sphenolobus Steph. - Mem. Soc. Fauna Flora Fenn. 8: 282-297.

Buchbender, V., Hespanhol, H., Krug, M., Sérgio, C., Séneca, A., Mau, K., Hedenäs, L. and QuAndt, D. (2014): Phylogenetic reconstructions of the Hedwigiaceae reveal cryptic speciation and hybridisation in Hedwigia. - Bryoph. Div. Evol. 36: 1-21.

https://doi.org/10.11646/bde.36.1.1

Caparrós, R., Lara, F., Draper, I., Mazimpaka, V. and Garilleti, R. (2016): Integrative taxonomy sheds light on an old problem: the Ulota crispa complex (Orthotrichaceae, Musci).

- Bot. J. Linn. Soc. 180: 427-451. https://doi.org/10.1111/boj.12397

CASPARI, S. and Erzberger, P. (2019): 54. Tortella pseudofragilis (Thér.) Köckinger \& Hedenäs. - In: Ellis, L. T. (ed.): New national and regional bryophyte records, 58. - J. Bryol. 41(1): 63-84. https://doi.org/10.1080/03736687.2018.1559636

CASPARI, S., DÜRhAMmer, O., SAUER, M. and SChmidT, C. (2018): Rote Liste und Gesamtartenliste der Moose (Anthocerotophyta, Marchantiophyta und Bryophyta) Deutschlands. - In: METzing, D., Hofbauer, N., Ludwig, G. and MATZKe-Hajek, G. (eds): Rote Liste gefährdeter Tiere, Pflanzen und Pilze Deutschlands. Band 7: Pflanzen. Landwirtschaftsverlag, Münster. - Naturschutz und Biologische Vielfalt 70(7): 361-489.

DAmsholt, K. (1994): On the identity of Jungermannia groenlandica Nees. - J. Hattori Bot. Lab. 75: $173-178$.

DAmsholt, K. (2002): Illustrated flora of Nordic liverworts and hornworts. - Nordic Bryological Society, Lund.

De Roo, R. T., Hedderson, T. A. and Söderström, L. (2007): Molecular insights into the phylogeny of the leafy liverwort family Lophoziaceae Cavers. - Taxon 56: 301-314.

Deme, J., Csiky, J. and Erzberger, P. (2015): Campylopus fragilis (Brid.) Bruch \& Schimp. - In: Ellis, L. T. (ed.): New national and regional bryophyte records, 43. - J. Bryol. 37: 128-147. https://doi.org/10.1179/1743282015Y.0000000003

ECKel, P. (1998): A re-evaluation of Tortella (Musci, Pottiaceae) in conterminous USA and Canada, with a treatment of the European species Tortella nitida. - Bull. Buffalo Soc. Nat. Sci. 36: 117-191.

ECKel, P. (2010): Tortella bambergeri in North America and an evaluation of its taxonomy. - Bull. Buffalo Soc. Nat. Sci. 39: 1-10.

Eckstein, J., Nagy, J. and Erzberger, P. (2017): 26. Sciuro-hypnum curtum (Lindb.) Ignatov. - In: Ellis, L. T. (ed.): New national and regional bryophyte records, 53. - J. Bryol. 39(4): 368-387. https://doi.org/10.1080/03736687.2017.1384204.

Ellis, L. T. and PRICE, M. J. (2015): Review of the type specimens of species described by J. Hedwig in Phascum Hedw. (Pottiaceae). - J. Bryol. 37: 23-41. https://doi.org/10.1179/1743282014y.0000000116

Erzberger, P. (2009a): The genera Grimmia and Coscinodon (Grimmiaceae, Musci) in Hungary - Studia bot. hung. 40: 37-124.

Erzberger, P. (2009b): 15. Pseudoleskea incurvata (Hedw.) Loeske. - In: Blockeel, T. L. (ed.): New national and regional bryophyte records, 20. - J. Bryol. 31: 54-62. 
https://doi.org/10.1179/jbr.2009.31.1.64

ERzBERgER, P. (2014): 14. Encalypta rhaptocarpa Schwaegr. - In: Ellis, L. T. (ed.): New national and regional bryophyte records, 39. - J. Bryol. 36: 134-151.

https://doi.org/10.1179/1743282014Y.0000000100

Erzberger, P. (2015): 7. Bryum barnesii J. B. Wood ex Schimp. - In: Ellis, L. T. (ed.): New national and regional bryophyte records, 45. - J. Bryol. 37: 308-329.

https://doi.org/10.1179/1743282015Y.0000000035

Erzberger, P. (2016a): 23. Fissidens crispus Mont. - In: Ellis, L. T. (ed.): New national and regional bryophyte records, 48. - J. Bryol. 38: 243-244.

https://doi.org/10.1080/03736687.2016.1206685

Erzberger, P. (2016b): The genus Fissidens Hedw. (Bryophyta) in Hungary. - Studia bot. hung. 47: 41-139. https://doi.org/10.17110/StudBot.2016.47.1.41

Erzberger, P. (2018): 33. Pellia neesiana (Gottsche) Limpr. - In: Ellis, L. T. (ed.): New national and regional bryophyte records, 54. - J. Bryol. 40: 74-97.

https://doi.org/10.1080/03736687.2018.1425573

Erzberger, P. and Meinunger, L. (2014a): 8. Cephaloziella varians (Gottsche) Stephani var. varians. - In: Ellis, L. T. (ed.): New national and regional bryophyte records, 39. - J. Bryol. 36: 134-151. https://doi.org/10.1179/1743282014Y.0000000100

Erzberger, P. and Meinunger, L. (2014b): 27. Scapania praetervisa Meyl. - In: Ellis, L. T. (ed.): New national and regional bryophyte records, 39. - J. Bryol. 36: 134-151. https://doi.org/10.1179/1743282014Y.0000000100

Erzberger, P. and NÉmeth, C. (2014): 7. Campylopus flexuosus (Hedw.) Brid. - In: Ellis, L. T. (ed.): New national and regional bryophyte records, 39. - J. Bryol. 36: 134-151.

https://doi.org/10.1179/1743282014Y.0000000100

Erzberger, P. and Németh, Cs. (2015): 13. Campylium decipiens (Warnst.) Walsem. - In: Ellis, L. T. (ed.): New national and regional bryophyte records, 45. - J. Bryol. 37: 308-329. https://doi.org/10.1179/1743282015Y.0000000035

Erzberger, P. and PAPP, B. (2004): Annotated checklist of Hungarian bryophytes. - Studia bot. bung. 35: 91-149.

Erzberger, P. and PAPp, B. (2018): Tortella fasciculata and T. pseudofragilis (Pottiaceae, Bryophyta) in Hungary. - Studia bot. hung. 49(2): 39-48.

https://doi.org/10.17110/StudBot.2018.49.2.39

Erzberger, P. and Schröder, W. (2008): The genus Schistidium (Grimmiaceae, Musci) in Hungary. - Studia bot. hung. 39: 27-88.

ERzberger, P. and Schröder, W. (2013): The genus Bryum (Bryaceae, Musci) in Hungary. Studia bot. hung. 44: 5-192.

ERzberger, P. and SCHRöder, W. (2016): 4. Bryum tenuisetum Limpr. - In: Ellis, L. T. (ed.): New national and regional bryophyte records, 47. - J. Bryol. 38: 151-167.

https://doi.org/10.1080/03736687.2016.1171453

Erzberger, P., Baráth, K., and Gallego, M. T. (2018a): 37. Syntrichia ruralis (Hedw.) F. Weber \& D. Mohr var. epilosa (Venturi) J. J. Amann. - In: ElLIS, L. T. (ed.): New national and regional bryophyte records, 57. - J. Bryol. 40(4): 399-419.

https://doi.org/10.1080/03736687.2018.1523601

Erzberger, P., BednAREK-Ochyra, H. and Ochyra, R. (2016a): Grimmiaceae subfam. Racomitrioideae (Bryophyta) in Hungary. - Polish Bot. J. 61(1): 23-51.

https://doi.org/10.1515/pbj-2016-0015 
Erzberger, P., Németh, Cs., Baráth, K. and Mesterházy, A. (2018b): 45. Sematophyllum adnatum (Michx.) E. Britton. - In: Ellis, L. T. (ed.): New national and regional bryophyte records, 54. - J. Bryol. 40: 74-97. https://doi.org/10.1080/03736687.2018.1425573

Erzberger, P., NÉmeth, Cs. and Mesterházy, A. (2016b): 6. Callicladium haldanianum (Grev.) H. A. Crum. - In: Ellis, L. T. (ed.): New national and regional bryophyte records, 47. - J. Bryol. 38: 151-167. https://doi.org/10.1080/03736687.2016.1171453

Frahm, J.-P. and Walsemann, E. (1973): Nachträge zur Moosflora von Schleswig-Holstein. Mitteil. Arbeitsgem. Geobot. Schleswig-Holstein und Hamburg 23: 1-203.

Gallego, M. T., Hugonnot, V. and Cano, M. J. (2018): Taxonomic resurrection of an awnless variety of Syntrichia ruralis and comparison with other European muticous taxa in this genus. - J. Bryol. 40: 244-250. https://doi.org/10.1080/03736687.2018.1468971

Grolle, R. and Long, D. G. (2000): An annotated check-list of the Hepaticae and Anthocerotae of Europe and Macaronesia. - J. Bryol. 22: 103-140. https://doi.org/10.1179/jbr.2000.22.2.103

Grolle, R. and So, M. L. (2003): Riccia fruticulosa O. F. Müll., 1782 and blue Metzgeria (Marchantiophyta) in Europe. - Bot. J. Linn. Soc. 142: 229-235. https://doi.org/10.1046/j.1095-8339.2003.00176.x

Guerra, J., Cano, M. J. and Ros, R. M. (eds) (2006): Flora Briofítica Ibérica. Vol. III. Pottiales: Pottiaceae, Encalyptales: Encalyptaceae. - Universidad de Murcia, Sociedad Española de Briología, Murcia.

Hassel, K., Kyrkjeeide, M. O., Yousefi, N., Prestø, T., Stenøien, H. K., Shaw, J. A. and FLATBERG, K. I. (2018): Sphagnum divinum (sp. nov.) and S. medium Limpr. and their relationship to S. magellanicum Brid. - J. Bryol. 40: 197-222. https://doi.org/10.1080/03736687.2018.1474424

Hedenäs, L. (1994): The Hedwigia ciliata complex in Sweden, with notes on the occurrence of the taxa in Fennoscandia. - J. Bryol. 18: 139-157. https://doi.org/10.1179/jbr.1994.18.1.139

HedenÄs, L., Heinrichs, J. and Gallego, M. T. (2019): The Scandinavian Syntrichia ruralis complex (Musci. Pottiaceae): a chaos of diversification. - Plant Syst. Evol. 305: 639-661. https://doi.org/10.1007/s00606-019-01596-0

Hill, M. O., Bell, N., Bruggeman-Nannenga, M. A., Brugués, M., Cano, M. J., Enroth, J., Flatberg, K. I., Frahm, J.-P., Gallego, M. T., Garilleti, R., Guerra, J., Hedenäs, L., Holyoak, D. T., Hyvönen, J., Ignatov, M. S., Lara, F., Mazimpaka, V., Muñoz, J. and SÖDERSTRÖM, L. (2006): An annotated checklist of the mosses of Europe and Macaronesia. - J. Bryol. 28: 198-267. https://doi.org/10.1179/174328206x119998

Hodgetts, N., CÁlix, M. et al. (2019): A miniature world in decline: European red list of mosses, liverworts and hornworts. - IUCN, Brussels.

Hodgetts, N. G., Söderström, L., Blockeel, T. L., Caspari, S., Ignatov, M. S., Konstantinova, N. A., Lockhart, N., Papp, B., Schröck, C., Sim-Sim, M., Bell, D., Bell, N. E., Blom, H. H., Bruggeman-Nannenga, M. A., Brugués, M., Enroth, J., Flatberg, K. I., Garilleti, R., Hedenäs, L., Holyoak, D. T., Hugonnot, V., Kariyawasam, I., Köckinger, H., Kučera, J., LARA, F. and Porley, R. D. (2020): An annotated checklist of bryophytes of Europe, Macaronesia and Cyprus. - J. Bryol. 42(1): 1-116.

https://doi.org/10.1080/03736687.2019.1694329

Hofmann, H. (1998): A monograph of the genus Homalothecium (Brachytheciaceae, Musci). Lindbergia 23: 119-159.

Holyoak, D. T. (2003): A taxonomic review of some British coastal species of the Bryum bicolor complex, with a description of Bryum dyffrynense sp. nov. - J. Bryol. 25: 107-113. https://doi.org/10.1179/037366803235001779 
Holyoak, D. T. (2010): Notes on taxonomy of some European species of Ephemerum (Bryopsida: Pottiaceae). - J. Bryol. 32: 122-132. https://doi.org/10.1179/037366810x12578498136192

Holyoak, D. T. and HedenÄs, L. (2006): Morphological, ecological and molecular studies of the intergrading taxa Bryum neodamense and B. pseudotriquetrum (Bryopsida: Bryaceae). - J. Bryol. 28: 299-311. https://doi.org/10.1179/174328206x136304

Holz, I. (2005): Riccia. - In: Nebel, M. and Philip Pi, G. (eds): Die Moose Baden-Württembergs. Bd. 3. Ulmer, Stuttgart, pp. 116-138.

Hugonnot, V. (2010): Towards an improved understanding of Riccia ciliata Hoffm. (Marchantiopsida: Ricciaceae). - J. Bryol. 32: 300-303. https://doi.org/10.1179/jbr.2010.32.4.300

Ignatov, M. S. and Milyutina, I. A. (2007): On Sciuro-hypnum oedipodium and S. curtum (Brachytheciaceae, Bryophyta). - Arctoa 16: 47-61. https://doi.org/10.15298/arctoa.16.06

Ignatova, E. A., Kuznetsova, O. I., Fedosov, V. E. and Ignatov, M. S. (2016): On the genus Hedwigia (Hedwigiaceae, Bryophyta) in Russia. - Arctoa 25: 241-277. https://doi.org/10.15298/arctoa.25.20

Jovet-Ast, S. (1986): Les Riccia de la région Méditerranéenne. - Cryptogamie, Bryol. Lichénol. 7 : 287-431.

Jovet-Ast, S. (2000): Documents pour la connaissance des Riccia australiens (Hépatiques, Marchantiales) - nouvelles récoltes. Taxons nouveaux. Commentaires morphologiques \& écologiques. (Contribution to the knowledge of Australian Riccia (Hepaticae, Marchantiales): new records, new taxa, morphological and ecological data). - Cryptogamie, Bryol. 21: 289-343. https://doi.org/10.1016/s1290-0796(00)01042-7

Konstantinova, N. A., Ротеmkin, A. D. and Schlja Kov, R. N. (1992): Checklist of the Hepaticae and Anthocerotae of the former USSR. - Arctoa 1: 87-127. https://doi.org/10.15298/arctoa.01.02

Konstantinova, N. A., Bakalin, V. A., Andreeva, E. N., Bezgodov, A. G., Borovichev, E. A., Dulin, M. A. and Mamontov, Y. S. (2009): Checklist of liverworts (Marchantiophyta) of Russia. - Arctoa 18: 1-64. https://doi.org/10.15298/arctoa.18.01

Koponen, T. and Isovirta, P. (2010): Philonotis capillaris Lindb. and P. arnellii Husn.; one moss, two names. - Cryptogamie, Bryol. 31: 75-94.

KošnaR, J. and KolÁ ̌̌, F. (2009): A taxonomic study of selected European taxa of the Tortula muralis (Pottiaceae, Musci) complex: variation in morphology and ploidy level. - Preslia 81 : $399-421$.

KöCKINGER, H. (2017): Die Horn- und Lebermoose Österreichs (Anthocerotophyta und Marchantiophyta). - Catalogus Florae Austriae II(2): 1-382.

KöCKIngeR, H. and Heden Äs, L. (2017): A farewell to Tortella bambergeri (Pottiaceae) as understood over the last decades. - J. Bryol. 39: 213-225. https://doi.org/10.1080/03736687.2017.1307313

Köckinger, H. and KučERA, J. (2016): Brachythecium funkii Schimp. and B. japygum (Glow.) Köckinger \& Jan Kučera comb. nov., two alpine species hitherto included in B. cirrhosum (Schwägr.) Schimp. - J. Bryol. 38: 267-285. https://doi.org/10.1080/03736687.2016.1156355

Köckinger, H., Kučera, J., Hofmann, H., Müller, N. and Amann, G. (2010): Barbula consanguinea discovered in Switzerland and Austria, with a revision of former European records of B. indica. - Herzogia 25: 61-70. https://doi.org/10.13158/heia.25.1.2010.61

Köckinger, H., Suanjak, M., Schriebl, A. and Schröck, C. (2008): Die Moose Kärntens. Sonderreihe Natur Kärnten 4, Verlag des Naturwissenschaftlichen Vereins für Kärnten, Klagenfurt.

KuČera, J., Blockeel, T. L., Erzberger, P., Papp, B., Soldán, Z., Vella K, K., Werner, O. and Ros, R. M. (2018): The Didymodon tophaceus complex (Pottiaceae, Bryophyta) revisited: 
new data support the subspecific rank of currently recognized species. - Cryptogamie, Bryol. 39(2): 241-257. https://doi.org/10.7872/cryb/v39.iss2.2018.241

KuČera, J., Košnar, J. and Werner, O. (2013): Partial generic revision of Barbula (Musci: Pottiaceae): re-establishment of Hydrogonium and Streblotrichum, and the new genus Gymnobarbula. - Taxon 62: 21-39. https://doi.org/10.1002/tax.621004

KuČera, J., Kuznetsova, O. I., Manukjanová, A. and Ignatov, M. S. (2019): A phylogenetic revision of the genus Hypnum. Towards completion. - Taxon 68: 628-660. https://doi.org/10.1002/tax.12095

Latzel, A. (1930): Moose aus dem Komitate Vas u. einigen anderen Komitaten. - Magyar Bot. Lapok 29: 105-138.

MeINUNGer, L. and SCHRöder, W. (2007): Verbreitungsatlas der Moose Deutschlands. - O. Dürhammer für die Regensburgische Botanische Gesellschaft, Regensburg.

Mesterházy, A. and Németh, Cs. (2015): 6. Bruchia flexuosa (Schwaegr.) Müll. Hal. - In: Ellis, L. T. (ed.): New national and regional bryophyte records, 45. - J. Bryol. 37: 308-329. https://doi.org/10.1179/1743282015Y.0000000035

Mesterházy, A., BArÁth, K. and Erzberger, P. (2017): 26. Rhabdoweisia crispata (Dicks. ex With.) Lindb. - In: Ellis, L. T. (ed.): New national and regional bryophyte records, 50. - J. Bryol. 39: 99-114. https://doi.org/10.1080/03736687.2016.1259931

MülLER, K. (1954): Die Lebermoose Europas (Dr. L. Rabenhorst's Kryptogamen-Flora von Deutschland, 3. Aufl.) 6. Band, 4. Lieferung. - Akademische Verlagsgesellschaft, Leipzig, pp. 481-640.

NAGY, J. and Erzberger, P. (2018): Crossidium squamiferum (Viv.) Jur. - In: Ellis, L. T. (ed.): New national and regional bryophyte records, 55. - J. Bryol. 40(2): 173-187. https://doi.org/10.1080/03736687.2018.1454161

NAGY, J. and NÉmet h, Cs. (2017): A Pseudoleskea saviana (De Not.) Latzel lombosmoha Magyarországon. (Distribution of Pseudoleskea saviana (De Not.) Latzel in Hungary). - Kitaibelia 22(2): 272-285. https://doi.org/10.17542/kit.22.272

Nagy, J., Pap-Kovács, A. and Erzberger, P. (2019): Bibliography of bryological research in Hungary (1968-2018). - Studia bot. hung. 50(1): 53-106.

https://doi.org/10.17110/StudBot.2019.50.1.53.

Nebel, M. and Philippi, G. (eds) (2001): Die Moose Baden-Württembergs. Bd. 2. - Ulmer, Stuttgart.

NÉmeth, Cs. (2016): 20. Ditrichum lineare (Sw.) Lindb. - In: Ellis, L. T. (ed.): New national and regional bryophyte records, 48. - J. Bryol. 38: 235-259. https://doi.org/10.1080/03736687.2016.1206685

Németh, Cs. and NAGY, J. (2016): 4. Barbilophozia hatcheri (Evans) Loeske. - In: Ellis, L. T. (ed.): New national and regional bryophyte records, 49. - J. Bryol. 38: 327-347. https://doi. org/10.1080/03736687.2016.1225777

Németh, Cs., Kovács, A. and Erzberger, P. (2016): 30. Seligeria acutifolia Lindb. - In: Ellis, L. T. (ed.): New national and regional bryophyte records, 49. - J. Bryol. 38: 327-347. https://doi.org/10.1080/03736687.2016.1225777

Ódor, P. and Szurdoki, E. (2011): 8. Hookeria lucens (Hedw.) Sm. - In: Ellis, L. T. (ed.): New national and regional bryophyte records, 26. - J. Bryol. 33: 66-73. https://doi.org/10.1179/1743282010Y.0000000014

Orbán, S. and VAjDA, L. (1983): Magyarország mohaflórájának kézikönyve. - Akadémiai Kiadó, Budapest, $518 \mathrm{pp}$.

PAPp, B. and Sinigla, M. (2017): 32. Zygodon forsteri (Dicks.) Mitt. - In: Ellis, L. T. (ed.): New national and regional bryophyte records, 52. - J. Bryol. 39: 285-304.

https://doi.org/10.1080/03736687.2017.1341752 
Papp, B., Erzberger, P., Lökös, L., Szurdoki, E., Németh, Cs., Buczkó, K., Höhn, M., AszALósné Balogh, R., Baráth, K., Matus, G., Pif Kó, D. and FARKas, E. (2020): Taxonomical and chorological notes 12 (126-136). - Studia bot. hung. 51(1): 77-98. https://doi.org/10.17110/StudBot.2020.51.1.77

Papp, B., Erzberger, P., Ódor, P., Hock, Zs., Szövényi, P., Szurdoki, E. and Tóth, Z. (2010): Updated checklist and red list of Hungarian bryophytes. - Studia bot. hung. 41: 31-59.

SAUKEL, J. (1985): Zum Merkmalsbestand einiger mitteleuropäischer Arten der Lebermoosgattung Lophozia (Dum.) Dum. (Sektion Lophozia). - Stapfia 14: 149-185.

Schlesak, S., Hedenäs, L., Nebel, M. and Quandt, D. (2018): Clearing a taxonomic dustbin: placing the European Hypnum species in a phylogenetic context! - Bryoph. Div. Evol. 40: 37-54. https://doi.org/10.11646/bde.40.2.3

Schlesak, S., Hedenäs, L., Nebel, M. and Quandt, D. (2019): Addendum to Hypnum subcomplanatum Hedenäs, Schlesak \& Quandt. - Bryoph. Div. Evol. 41: 1.

https://doi.org/10.11646/bde.41.1.1

Schlja kov, R. N. (1970): [Sectio nova generis Lophozia Dum. emend. Loeske]. - Nov. sist. nizsh. rast. $7: 324-333$.

Schljakov, R. N. (1975): [Notulae systematicae de hepaticis s. str.]. - Nov. sist. nizsh. rast. 12: 307-317.

SchlJakov, R. N. (1980): Pechenochnye Mkhi Severa SSSR. 3. (Hepaticae of the north of the USSR. 3). - Nauka, Leningrad.

Schljakov, R. N. (1998): On the Lophozia groenlandica (Nees) Macoun (Hepaticae). - Arctoa 7: 191-196. https://doi.org/10.15298/arctoa.07.16

Schröder, W. and Erzberger, P. (2012): 4. Bryum subapiculatum Hampe. - In: Ellis, L. T. (ed.): New national and regional bryophyte records, 31. - J. Bryol. 34: 123-134. https://doi.org/10.1179/1743282012Y.0000000009

SMITH, A.J. E. (1990): The liverworts of Britain and Ireland. - Cambridge University Press, Cambridge.

Söderström, L., Hagborg, A., von Konrat, M., Bartholomew-Began, S., Bell, D., Briscoe, L., Brown, E., Cargill, D. C., Costa, D. P., Crandall-Stotler, B. J., et al. (2016): World checklist of hornworts and liverworts. - PhytoKeys 59: 1-828.

Söderström, L., URmi, E. and VÁŇA, J. (2002): Distribution of Hepaticae and Anthocerotae in Europe and Macaronesia. - Lindbergia 27: 3-47.

Spence, J. R. (2014): Bryaceae. - In: Flora of North America. Vol. 28. Bryophyta, Part 2. Oxford University Press, New York.

ŞTEFĂNUT,, S. (2008): The hornwort and liverwort atlas of Romania. - Ars Docendi, Bucharest.

Szücs, P. and Bidló, A. (2012): 10. Orthodontium lineare Schwägr. - In: Ellis, L. T. (ed.): New national and regional bryophyte records, 33. - J. Bryol. 34: 281-291. https://doi.org/10.1179/1743282012Y.0000000030

Tóтн, Z. (1986): A Tortula Hedw. Sect. Rurales De Not. (Musci, Pottiaceae) rendszertani revíziója és elterjedése a Kárpát-medencében. (Taxonomic revision of Tortula Hedw. sect. Rurales De Not. (Musci, Pottiaceae) and its distribution in the Carpathian Basin). - Abstracta Bot. 10: $145-185$.

VÁŇA, J. and ENGEL, J. J. (2013): The liverworts and hornworts of the Tristan da Cunha group of islands in the south Atlantic Ocean. - Mem. New York Bot. Gard. 105: 1-138.

Vilnet, A. A., Konstantinova, N. A. and Troitsky, A. V. (2010): Molecular insight on phylogeny and systematics of the Lophoziaceae, Scapaniaceae, Gymnomitriaceae and Jungermanniaceae. - Arctoa 19: 31-50.

(submitted: 30.10 .2020 , accepted: 30.11 .2020 ) 
Appendix 1. Taxa not included in PAPP et al. (2010) (but inserted in the main checklist).

\subsection{Additions to the bryophyte flora of Hungary (taxa not known previously to occur in Hungary).}

\section{Liverworts}

Barbilophozia hatcheri (A. Evans) Loeske - NÉMETH and NAGY (2016).

Cephaloziella divaricata var. scabra (M. Howe) Haynes - (B-Erzberger 19052, det. Meinunger, unpublished) see entry in main list.

Cephaloziella varians (Gottsche) Steph. - Erzberger and Meinunger (2014a).

Pellia neesiana (Gottsche) Limpr. - ERzBERGER (2018).

Riccia glauca var. ciliaris Warnst. - see entry in main list.

Scapania praetervisa Meyl. - ERzBerger and Meinunger (2014b).

\section{Mosses}

Brachythecium olympicum (Jur.) Vanderp. et al. - PAPP et al. (2020).

Bruchia flexuosa (Schwägr.) Müll. Hal. - Mesterházy and NÉmet H (2015).

Bryum barnesii J. B. Wood - ERzBERGER (2015).

Callicladium haldanianum (Grev.) H. A. Crum - ERzBERger et al. (2016b).

Campylopus flexuosus (Hedw.) Brid. - ERZBERGER and NÉMETH (2014).

Campylopus fragilis (Brid.) Bruch et Schimp. - Deme et al. (2015).

Codonoblepharon forsteri (Dicks.) Goffinet (Zygodon forsteri (Dicks.) Mitt.) - PAPP and Sinigla (2017).

Crossidium squamiferum (Viv.) Jur. var. squamiferum - NAGY and ERZBERGER (2018).

Didymodon tophaceus subsp. erosus (J. A. Jiménez et J. Guerra) Jan Kučera - KučERA et al. (2018).

Didymodon tophaceus subsp. sicculus (M. J. Cano, Ros, García-Zam. et J. Guerra) Jan Kučera KuČERA et al. (2018).

Ditrichum lineare (Sw.) Lindb. - NÉMETH (2016).

Encalypta rhaptocarpa Schwägr. - ERzBERGER (2014).

Fissidens crispus Mont. - ERzBerger (2016a).

Fissidens dubius var. mucronatus (Limpr.) Kartt., Hedenäs et L. Söderstr. - ERzBERGER (2016b).

Heterocladium heteropterum (Brid.) Schimp. - BARÁTH and ERZBERGER (2017a).

Hookeria lucens (Hedw.) Sm. - Ódor and SzURDoki (2011).

Imbribryum subapiculatum (Hampe) D. Bell et Holyoak - SCHRÖDER and ERzBERgER (2012).

Imbribryum tenuisetum (Limpr.) D. Bell et Holyoak - ERZBERGER and SCHRöDER (2016).

Orthodontium lineare Schwägr. - SzücS and BIDLÓ (2012).

Plagiothecium latebricola Schimp. - BARÁTH and ERZBERger (2017b).

Racomitrium microcarpum (Hedw.) Brid. - BEDNAREK-OCHYRA et al. (2011).

Rhabdoweisia crispata (Dicks.) Lindb. - MESTERHÁzy et al. (2017).

Sciuro-hypnum curtum (Lindb.) Ignatov - ECKSTEIN et al. (2017).

Seligeria acutifolia Lindb. - NÉMETH et al. (2016).

Seligeria patula (Lindb.) I. Hagen - a specimen collected by L. Vajda (BP) was revised by L. Gos, see note in main checklist.

Sematophyllum adnatum (Michx.) E. Britton - ERzBERGER et al. (2018b).

Sphagnum divinum Flatberg et Hassel - see entry in main list; specimens of this taxon were previously considered to represent $S$. magellanicum Brid. 
Syntrichia ruralis var. epilosa (Venturi) J. J. Amann - ERZBERGER et al. (2018a).

Thamnobryum neckeroides (Hook.) E. Lawton - see entry in main list.

Tortella fasciculata (Culm.) Culm. - Köckinger and Hedenäs (2017), Erzberger and PApP (2018).

Tortella pseudofragilis (Thér.) Köckinger et Hedenäs - CASPARI and Erzberger (2019), ErzBERGER and PAPP (2018).

Tortula acaulon var. papillosa (Lindb.) R. H. Zander (Phascum cuspidatum var. papillosum (Lindb.) G. Roth) - infraspecific taxon recognised in Hodgets et al. (2020) and recorded from Hungary (Erzberger et al. unpublished).

Ulota crispula Bruch - CAPARrós et al. (2016).

Ulota intermedia Schimp. - CAPARRós et al. (2016).

\subsection{Taxa previously known to occur in Hungary but not recognised in PAPP et al. (2010).}

\section{Liverworts}

Lophocolea coadunata (Sw.) Mont. - see entry in main list; this name replaces the familiar name Lophocolea bidentata (L.) Dumort., which stands for a different taxon.

Lophozia guttulata (Lindb. et Arnell) A. Evans - see entry in main list; specimens of this taxon were previously considered to represent $L$. longiflora (Nees) Schiffn.

Lophozia silvicola $\mathrm{H}$. Buch - see entry in main list; previously included in L. ventricosa (Dicks.) Dumort.

Scapania parvifolia Warnst. - see entry in main list; previously included in S. scandica (Arnell et H. Buch) Macvicar

\section{Mosses}

Atrichum flavisetum Mitt. - see entry in main list; previously included in A. undulatum (Hedw.) P. Beauv.

Campylium protensum (Brid.) Kindb. (Campylium stellatum var. protensum (Brid.) Bryhn) -upgraded to specific rank, previously included in C. stellatum (Hedw.) Lange et C. E. O. Jensen

Cynodontium strumiferum (Hedw.) Lindb. - upgraded to specific rank from C. polycarpon var. strumiferum

Hedwigia emodica Hampe ex Müll. Hal. (Hedwigia ciliata var. leucophaea Bruch et Schimp.) - see entry in main list; upgraded to specific rank

Hypnum cupressiforme var. lacunosum Brid. - see entry in main list; previously included in $\mathrm{H}$. cupressiforme Hedw.

Hypnum cupressiforme var. subjulaceum Molendo - see entry in main list; previously included in $H$. cupressiforme Hedw.

Orthotrichum schimperi Hammar - included in Orthotrichum pumilum Sw. as Orthotrichum pumilum var. schimperi (Hammar) Hinn. in BoRos (1968), upgraded to specific rank.

Palustriella falcata (Brid.) Hedenäs - upgraded to specific rank and recorded from Hungary (PAPP et al. 2020).

Polytrichum perigoniale Michx. - upgraded to specific rank and recorded from 5 floristical grid cells (Erzberger et al. unpublished).

Ptychostomum pseudotriquetrum var. bimum (Schreb.) Holyoak et N. Pedersen - previously included in Bryum pseudotriquetrum (Hedw.) P. Gaertn., E. Mey. et Scherb. (syn. Ptychostomum 
pseudotriquetrum J. R. Spence et H. P. Ramsay ex Holyoak et N. Pedersen) or treated as separate species Bryum bimum (Schreb.) Turner (ERZBERger and SCHRöDER 2013).

Streblotrichum convolutum var. commutatum (Jur.) J. J. Amann - infraspecific taxon recognised in HodgetTs et al. (2020) and recorded from 20 floristical grid cells (Erzberger et al. unpublished).

Syntrichia montana var. calva (Durieu et Sagot ex Bruch et Schimp.) J. J. Amann - previously included in S. montana Nees.

Syntrichia subpapillosissima (Bizot et R. B. Pierrot ex W. A. Kramer) M. T. Gallego et J. Guerra (Tortula papillosissima (Copp.) Broth. var. submamillosa (W. A. Kramer) Heinrichs et Caspari) see entry in main list; previously included in Tortula papillosissima (Copp.) Broth.

Tortula acaulon var. pilifera (Hedw.) R. H. Zander (Phascum cuspidatum var. piliferum (Hedw.) Hook. et Taylor) - see entry in main list; previously included in Phascum cuspidatum Hedw. (syn. Tortula acaulon (With.) R. H. Zander).

Tortula muralis subsp. muralis var. aestiva Hedw. - previously included in T. muralis L. ex Hedw., not rare in Hungary (BOROS 1968, ORBÁN and VAJDA 1983).

\section{Appendix 2. Taxa excluded from the checklist.}

\section{Liverworts}

Lophocolea bidentata (L.) Dumort. [Lophocolea cuspidata (Nees) Limpr.] - see entry in main list under L. coadunata (Sw.) Mont.

Lophozia longiflora (Nees) Schiffn. [Lophozia ventricosa var. longiflora (Nees) Macoun, Lophozia ventricosa var. uliginosa auct. (sensu SöDERSTRÖm, URmi, et al. 2002, DAMSholt 2002)] (Lophozia porphyroleuca (Nees) Schiffn.) - The Hungarian specimens under this name were collected on dead wood (in one case on peat with Leucobryum glaucum), they were revised to L. guttulata (see the entry in main list under that name).

Metzgeria simplex Lorb. ex Müll. Frib. [Metzgeria conjugata subsp. simplex (Lorb. ex Müll. Frib.) R. M. Schust.] - doubtful according to ERzBerger and PAPP (2004). To our knowledge the only voucher specimen for this taxon has not been examined cytologically, which would be necessary to identify it properly as the haploid taxon, since it cannot reliably be distinguished from $M$. conjugata by morphological methods. Until appropriate material of this taxon turns up, we exclude Metzgeria simplex from the Hungarian bryophyte flora.

Metzgeria violacea (Ach.) Dumort. [Metzgeria fruticulosa auct. non (O. F. Müll.) A. Evans] 'Grolle and So (2003) demonstrated that the name Metzgeria fruticulosa technically belongs to Riccardia palmata and Metzgeria violacea is the name that should be used' (HoDGeTTs et al. (2020): annotation 127). According to ERZBERGER and PAPP (2004), no voucher specimen could be located for the single record of this taxon, which was already considered doubtful by Boros (1968). We therefore exclude this taxon from the bryophyte flora of Hungary until proper material turns up.

Riccia crystallina L. emend. Raddi - All reports of this species from Hungary are referable to Riccia cavernosa Hoffm. emend. Raddi. R. crystallina does not occur in Central Europe (Holz 2005).

Riccia crinita Taylor - See the note under Riccia ciliata Hoffm.

Riccia duplex Lorb. ex Müll. Frib. - According to Meinunger and Schröder (2007), R. duplex is only a cytological race (with doubled chromosome number) of $R$. canaliculata Hoffm. We therefore follow CASPARI et al. (2018) and include $R$. duplex in $R$. canaliculata. In addition, the only Hungarian specimen (for details, see ERZBERGER and PAPP 2004) was interpreted differently by L. Vajda and S. Jovet-Ast, who revised Vajda's determination to $R$. canaliculata. 


\section{Mosses}

Aloina brevirostris (Hook. et Grev.) Kindb. - The specimens under this name in BP and in the herbarium of the first author do not represent this species (B. Papp and P. Erzberger, unpublished revision).

Brachythecium tenuicaule (Spruce) Kindb. (Rhynchostegiella tenuicaulis (Spruce) Kartt., Cirriphyllum germanicum (Grebe) Loeske et M. Fleisch.) - We follow CASPARI et al. 2018 and NeBEL and PHILIPPI (2001) and include Brachythecium tenuicaule in the synonymy of $B$. tommasinii (Sendtn. ex Boulay) Ignatov et Huttunen.

Bryum neodamense Itzigs. ex Müll. Hal. - See the note under Ptychostomum pseudotriquetrum (Hedw.) J. R. Spence et H. P. Ramsay ex Holyoak et N. Pedersen.

Bryum versicolor A. Braun ex Bruch et Schimp. - No specimens to support earlier reports of this species in Hungary were found during generic revision (ERZBERGER and SCHRÖDER 2013).

Ceratodon conicus (Hampe) Lindb. [Ceratodon purpureus var. conicus (Hampe) Husn.] -The report by ZANTEN (1999) is based on a single sterile specimen (ERZBEREGER and PAPP 2004), however, the diagnostic difference to C. purpureus is found in the peristome. Since the specimen collected by Zanten is the only voucher for the taxon in Hungary, we consider the presence of $C$. conicus doubtful and exclude it from the Hungarian bryophyte flora until appropriate material turns up.

Dialytrichia mucronata (Brid.) Broth. - According to ERZBERGER and PAPP (2004), the presence of this species in Hungary was based on a single collection by A. Latzel (BP 110580. Com. Sopron. Kéthely [Répcekéthely = Mannersdorf an der Rabnitz]. In der Klausen. 25.04.1895. leg. A. Latzel). This record was also published by LATzel (1930). However, the locality 'Klausen' is situated in present-day Austria (H. Köckinger and Chr. Schröck, pers. comm.). Therefore, $D$. mucronata must be excluded from the Hungarian bryoflora.

Fissidens crassipes subsp. warnstorfii (M. Fleisch.) Brugg.-Nann. - See the note under F. crassipes.

Fissidens curnovii Mitt. (F. bryoides var. caespitans Schimp.) - No specimens to support earlier reports of this species in Hungary were found during generic revision, the specimen cited in ERzBERger and PAPP (2004) was revised to F. bryoides var. bryoides (ERzBERGER 2016b).

Fissidens exiguus Sull. - see entry in Appendix 3.

Lescuraea incurvata (Hedw.) E. Lawton (Pseudoleskea incurvata (Hedw.) Loeske) - see entry in Appendix 3.

Orthotrichum scanicum Grönvall - A single specimen was found under this name in BP (Comit. Fejér. Ad corticem in silva Nagy-erdő prope Vajta, 14.05.1952, without collector, det. B. Oláh, rev. L. Vajda, BP 157967) and was revised to O. pallens by P. Erzberger and Th. Kiebacher in 2017 (unpublished).

Orthotrichum stellatum Brid. (O. braunii Bruch et Schimp.) - All specimens under this name in BP collected in Hungary (13 specimens: BP 10021, BP 39181, BP 57753, BP 57767, BP 58398, BP 58399, BP 58400, BP 59357, BP 59404, BP 62779, BP 63935, BP 63937, BP 156033) were revised to other species by P. Erzberger and Th. Kiebacher in 2017 (unpublished).

Orthotrichum tenellum Bruch ex Brid. - Two specimens were found under this name in BP collected in Hungary (BP 155951, BP 10043), both were revised to other species by Th. Kiebacher 12.10.2017 (unpublished).

Pterygoneurum compactum M. J. Cano, J. Guerra et Ros - see the note under Pterygoneurum ovatum (Hedw.) Dixon.

Pterygoneurum crossidioides W. Frey, Herrnst. et Kürschn. - see the note under Pterygoneurum ovatum (Hedw.) Dixon.

Pterygoneurum squamosum Segarra et Kürschn. - see the note under Pterygoneurum ovatum (Hedw.) Dixon. 
Ptychostomum bornholmense (Wink. et R. Ruthe) Holyoak et N. Pedersen (Bryum bornholmense Wink. et R. Ruthe) - There is no specimen to support earlier reports of this species in Hungary (ERZBERGER and SCHRÖDER 2013).

Ptychostomum schleicheri (DC.) J. R. Spence ex D. Bell et Holyoak (Bryum schleicheri DC.) - All specimens originally labelled Bryum schleicheri were revised to B. pseudotriquetrum (ERZBERGER and SCHRÖDER 2013).

Racomitrium obtusum (Brid.) Brid. - All specimens under this name in BP collected in Hungary were shown by $\mathrm{H}$. Bednarek-Ochyra to represent epilose morphs of Bucklandiella heterosticha (Hedw.) Bednarek-Ochyra et Ochyra (Racomitrium heterostichum (Hedw.) Brid.) (ERzBERGER et al. 2016a).

Sphagnum magellanicum Brid. - see the note under Sphagnum divinum Flatberg et Hassel.

Tortella bambergeri auct. europ. non (Schimp.) Broth. - see the notes under Tortella fasciculata (Culm.) Culm., Tortella pseudofragilis (Thér.) Köckinger et Hedenäs and Tortella tortuosa (Hedw.) Limpr.

Tortula papillosissima (Copp.) Broth. var. papillosissima (Syntrichia papillosissima (Copp.) Loeske) - see the note under Syntrichia subpapillosissima (Bizot et R. B. Pierrot ex W. A. Kramer) M. T. Gallego et J. Guerra. The corresponding specimens from BP cited in Тóтн (1986) were revised by U. Abts (unpublished).

\section{Appendix 3. Problematic taxa (not included in main list).}

\section{Liverworts}

Chiloscyphus pallescens var. fragilis (Roth) Müll. Frib. - see annotation 81 in HodGETTs et al. 2020: 'The Chiloscyphus polyanthos complex has been treated in various ways in the past, without any recent solution. Some authors have treated it as one species (Chiloscyphus polyanthos) with two subspecies (subsp. polyanthos and pallescens (e.g. SMITH 1990); others as four segregate species (Chiloscyphus polyanthos, Chiloscyphus pallescens, Chiloscyphus fragilis and Chiloscyphus rivularis (e.g. Konstantinova, Potemkin, et al. 1992). Grolle and Long (2000) kept two species, but did not deal with any subspecific taxa. SöDERSTRöm, URMI, et al. (2002) kept two species but recognised four varieties (Chiloscyphus polyanthos var. polyanthos and var. rivularis and Chiloscyphus pallescens var. pallescens and var. fragilis). KoNSTANTINOVA, BAKALIN, et al. (2009) also used this concept as well as the World checklist of hornworts and liverworts (SÖDERSTRÖM et al. 2016). However, several recent publications do not recognise any varieties of the two species (e.g. KöcKINGER 2017). The distribution of the taxa recognised here is therefore not recorded consistently over the continent.' Var. fragilis is distinguished in Boros (1968), but the distinction from var. pallescens seems difficult (DAMShOlt 2002). We follow CASPARI et al. (2018) in not distinguishing between varieties. Chiloscyphus polyanthos var. rivularis (Schrad.) Nees - see also the note on Chiloscyphus pallescens var. fragilis. Var rivularis is distinguished in Boros (1968), but we follow CASPARI et al. 2018 and many other authors and do not recognise this variety.

\section{Mosses}

Abietinella abietina var. hystricosa (Mitt.) Sakurai - On a joint excursion of the authors with T. Pócs and Cs. Németh in 2014, a population of Abietinella abietina (Hedw.) M. Fleisch. was studied in Aggtelek National Park (Borsod-Abaúj-Zemplén County 7490.3 Bódvarákó, limestone grassland on Esztrámos Hill, N 48 31' 01.0”, E 2044' 50,4”, 300 m a.s.l., 18.03.2014, 
leg. Erzberger, Németh, Papp and Pócs, B-Erzberger 17082 dupl. in BP and EGR). There was a discussion whether these plants belong to var. abietina or var. hystricosa, and a sample was sent to Nick Hodgetts for revision together with other collections of T. Pócs from Romania. The answer 'I agree that all the specimens key out as hystricosa, although your specimen from Esztramos is the least satisfactory. It just emphasises the unsatisfactory nature of these two taxa' did not finally resolve the question. T. Pócs and B. Papp supported acceptance as var. hystricosa, whereas P. Erzberger's opinion was that intermediate forms between the two varieties were at hand. At the moment, this taxon remains controversial.

Campylium decipiens (Warnst.) Walsem. - See the note under Drepanocladus polygamus. ERzBERGER and NÉmETH (2015) reported C. decipiens (det. Meinunger) from Hungary, but that report remains controversial, since a specimen shown to L. Hedenäs was revised by him to Pseudocampylium radicale (unpublished). The population at the original site had been destroyed by wild animals. Until proper material turns up, we exclude this taxon as doubtful from the Hungarian checklist.

Fissidens bambergeri Schimp. ex Milde - although provisionally recognised in ERZBERGER (2016b), we do not include it in this updated checklist pending further studies. The same applies for $F$. exiguus Sull. See also annotation 217 in HodgetTs et al. (2020): 'The status of Fissidens bambergeri and Fissidens exiguus, treated here as synonyms of Fissidens viridulus, remains problematic. In a recent DNA study, it appeared that an incompletely limbate species of the Fissidens bryoides complex from the Netherlands was different from Fissidens viridulus (H. N. Siebel and M. Stech, pers. comm. 2019). However, several incompletely limbate species in this complex have been described. A morphological and molecular revision of these species is needed'.

Fissidens exiguus Sull. - see the note above under Fissidend bambergeri.

Homalothecium lutescens var. fallax (H. Philib. ex Schimp.) Düll - Although recognised in HodgETTS et al. (2020) and distinguished by Boros (1968), this taxon has been mostly neglected following Hofm ANN (1998). The only difference between var. fallax and var. lutescens is the shape and orientation of the capsule: straight and erect in var. fallax, and curved and inclined in the typical variety. However, these characters are not constant. Some authors assume a hybrid origin (between H. lutescens var. lutescens and H. sericeum, HofmanN 1998). We follow CASPARI et al. (2018) in not recognising infraspecific taxa.

Lescuraea incurvata (Hedw.) E. Lawton (syn. Pseudoleskea incurvata (Hedw.) Loeske) - In ERZBERGER and PAPP (2004) this species was excluded on the basis of revised specimens. However, meanwhile other specimens had turned up (e.g. ERZBERGER 2009b), but these were ultimately revised to $P$. saviana (for details, see NAGY and NÉMETH 2017). The only remaining evidence for the presence of $L$. incurvata in Hungary is a report by LATZEL (1930) from the Köszeg Mts without a specimen. K. Baráth and P. Erzberger searched the valley described by Latzel for the species in 2016 without finding it. In view of the difficulties of the distinction between $P$. saviana and $L$. incurvata we cannot accept Latzel's report without a specimen as sufficient evidence and therefore exclude $L$. incurvata until proper material is found.

Mnium marginatum var. dioicum (H. Müll.) Crundwell - Although recognised in HodgetTs et al. (2020), the taxonomy of this and related taxa still seems problematic, therefore we follow CASPARI et al. (2018) in not recognising infraspecific taxa of $M$. marginatum. See also ERZBERGER and PAPP (2004).

Ptychostomum badium (Bruch ex Brid.) J. R. Spence (Bryum badium (Bruch ex Brid.) Schimp.) not recognised in HODGETTs et al. (2020), doubtfully recorded from Hungary (ERZBERGER and SCHRÖDER 2013).

Ptychostomum knowltonii (Barnes) J. R. Spence (Bryum knowltonii Barnes) - doubtfully recorded from Hungary (ERZBERGER and SCHRÖDER 2013). 
Ptychostomum kunzei (Hornsch.) J. R. Spence (Bryum kunzei Hornsch.) - due to the difficulties in separating this taxon from Ptychostomum imbricatulum (Müll. Hal.) Holyoak et N. Pedersen (Bryum caespiticium Hedw.) many authors have included the former in the synonymy or as an infraspecific taxon in the latter (ERzBERGER and SCHRöDER 2013). We follow KöcKINGER et al. (2008) and include P. kunzei as a taxon of controversial status (doubtful infraspecific rank or representing merely an environment-induced modification) in P. imbricatulum.

Ptychostomum lonchocaulon (Müll. Hal.) J. R. Spence (Bryum lonchocaulon Müll. Hal.) - the status of this taxon is controversial: accepted as species by MEINUNGER and SCHRÖDER (2007), considered conspecific with Bryum pallescens in HILL et al. (2006), not recognised by HoDGETTS et al. (2020). It has been shown to occur in Hungary (ERZBERGER and SCHRöDER 2013), however due to the difficulties in separating it from Ptychostomum pallescens s.str. (ERzBERgER and SCHRÖDER 2013), we include it in a broadly conceived Ptychostomum pallescens.

Appendix 4. Updated checklist of Hungarian bryophytes ordered alphabetically according to traditional nomenclature, PAPP et al. (2010), also list of synonyms.

In the following list, only taxa recognised in PAPP et al. (2010) are contained; excluded taxa are not listed, see Appendix 2 for these. For the taxa newly reported from Hungary, see Appendix 1.

The abbreviation p. p. (latin: pro parte) indicates that a name was used in PAPP et al. (2010) in a wider sense than the same name accepted in the present checklist (e. g. in the previous checklist, Ulota crispa contained, apart from $U$. crispa s. str., also the new segregates $U$. crispula and U. intermedia).

\section{Hornworts}

Anthoceros agrestis $=$ Anthoceros agrestis Paton

Phaeoceros carolinianus $=$ Phaeoceros carolinianus (Michx.) Prosk.

\section{Liverworts}

Anastrophyllum hellerianum $=$ Crossocalyx bellerianus (Nees ex Lindenb.) Meyl.

Anastrophyllum michauxii $=$ Anastrophyllum michauxii (F. Weber) H. Buch

Anastrophyllum minutum $=$ Sphenolobus minutus (Schreb. ex D. Crantz) Berggr.

Aneura pinguis = Aneura pinguis (L.) Dumort.

Apometzgeria pubescens $=$ Metzgeria pubescens (Schrank) Raddi

Asterella saccata $=$ Asterella saccata (Wahlenb.) A. Evans

Athalamia hyalina $=$ Clevea hyalina (Sommerf.) Lindb.

Barbilophozia barbata = Barbilophozia barbata (Schmidel ex Schreb.) Loeske

Barbilophozia floerkei = Neoorthocaulis floerkei (F. Weber et D. Mohr) L. Söderstr., De Roo et Hedd.

Bazzania trilobata = Bazzania trilobata (L.) Gray

Blasia pusilla = Blasia pusilla L.

Blepharostoma trichophyllum $=$ Blepharostoma trichophyllum (L.) Dumort.

Calypogeia azurea $=$ Calypogeia azurea Stotler et Crotz

Calypogeia fissa $=$ Calypogeia fissa (L.) Raddi

Calypogeia integristipula $=$ Calypogeia integristipula Steph . 
Calypogeia muelleriana = Calypogeia muelleriana (Schiffn.) Müll. Frib.

Calypogeia neesiana = Calypogeia neesiana (C. Massal. et Carestia) Müll. Frib.

Calypogeia suecica $=$ Calypogeia suecica (Arnell et J. Perss.) Müll. Frib.

Cephalozia bicuspidata = Cephalozia bicuspidata (L.) Dumort.

Cephalozia catenulata $=$ Fuscocephaloziopsis catenulata (Huebener) Váňa et L. Söderstr.

Cephalozia lacinulata = Cephalozia lacinulata (J. B. Jack ex Gottsche et Rabenh.) Spruce

Cephalozia lunulifolia $=$ Fuscocephaloziopsis lunulifolia (Dumort.) Váňa et L. Söderstr.

Cephalozia macrostachya = Fuscocephaloziopsis macrostachya (Kaal.) Váňa et L. Söderstr.

Cephalozia pleniceps = Fuscocephaloziopsis pleniceps (Austin) Váňa et L. Söderstr.

Cephaloziella divaricata $=$ Cephaloziella divaricata $(\mathrm{Sm}$.) Schiffn.

Cephaloziella hampeana $=$ Cephaloziella hampeana $($ Nees) Schiffn. ex Loeske

Cephaloziella integerrima $=$ Cephaloziella integerrima (Lindb.) Warnst.

Cephaloziella rubella $=$ Cephaloziella rubella $(\mathrm{Nees})$ Warnst.

Cephaloziella spinigera $=$ Cephaloziella spinigera (Lindb.) Jørg.

Cephaloziella stellulifera = Cephaloziella stellulifera (Taylor ex Carrington et Pearson) Croz.

Chiloscyphus pallescens = Chiloscyphus pallescens (Ehrh.) Dumort.

Chiloscyphus polyanthos = Chiloscyphus polyanthos $(\mathrm{L}$.) Corda

Cololejeunea calcarea $=$ Cololejeunea calcarea $(\mathrm{Lib}$.$) Steph$.

Cololejeunea rossettiana $=$ Cololejeunea rossettiana (C. Massal.) Schiffn.

Conocephalum conicum $=$ Conocephalum conicum (L.) Dumort.

Conocephalum salebrosum = Conocephalum salebrosum Szweyk., Buczk. et Odrzyk.

Diplophyllum albicans $=$ Diplophyllum albicans (L.) Dumort.

Diplophyllum obtusifolium = Diplophyllum obtusifolium (Hook.) Dumort.

Fossombronia foveolata $=$ Fossombronia foveolata Lindb.

Fossombronia pusilla = Fossombronia pusilla (L.) Nees

Fossombronia wondraczekii = Fossombronia wondraczekii (Corda) Dumort. ex Lindb.

Frullania dilatata $=$ Frullania dilatata $($ L.) Dumort.

Frullania fragilifolia $=$ Frullania fragilifolia (Taylor) Gottsche, Lindenb. et Nees

Frullania inflata $=$ Frullania cleistostoma Schiffn. et W. Wollny

Frullania jackii $=$ Frullania jackii Gottsche

Frullania tamarisci $=$ Frullania tamarisci $($ L.) Dumort.

Gymnocolea inflata $=$ Gymnocolea inflata (Huds.) Dumort.

Jamesoniella autumnalis = Syzygiella autumnalis (DC.) K. Feldberg, Váňa, Hentschel et Heinrichs Jungermannia atrovirens $=$ Jungermannia atrovirens Dumort.

Jungermannia caespiticia $=$ Endogemma caespiticia (Lindenb.) Konstant., Vilnet et A. V. Troitsky

Jungermannia gracillima $=$ Solenostoma gracillimum $(\mathrm{Sm}$.$) R. M. Schust.$

Jungermannia hyalina $=$ Solenostoma byalinum (Lyell) Mitt.

Jungermannia leiantha $=$ Liochlaena lanceolata Nees

Jungermannia pumila = Jungermannia pumila With.

Jungermannia sphaerocarpa = Solenostoma sphaerocarpum (Hook.) Steph.

Jungermannia subulata $=$ Liochlaena subulata (A. Evans) Schljakov

Leiocolea badensis = Mesoptychia badensis (Gottsche ex Rabenh.) L. Söderstr. et Váňa

Leiocolea collaris = Mesoptychia collaris (Nees) L. Söderstr. et Váňa

Leiocolea heterocolpos = Mesoptychia heterocolpos (Thed. ex Hartm.) L. Söderstr. et Váňa

Lejeunea cavifolia = Lejeunea cavifolia (Ehrh.) Lindb.

Lepidozia reptans $=$ Lepidozia reptans $(\mathrm{L}$.) Dumort.

Lophocolea bidentata $=$ Lophocolea coadunata (Sw.) Mont.

Lophocolea heterophylla $=$ Lophocolea heterophylla (Schrad.) Dumort. 
Lophocolea minor $=$ Lophocolea minor Nees

Lophozia ascendens $=$ Lophozia ascendens (Warnst.) R. M. Schust.

Lophozia bicrenata $=$ Isopaches bicrenatus (Schmidel ex Hoffm.) H. Buch

Lophozia excisa = Lophoziopsis excisa (Dicks.) Konstant. et Vilnet

Lophozia incisa $=$ Schistochilopsis incisa (Schrad.) Konstant.

Lophozia longidens = Lophoziopsis longidens (Lindb.) Konstant. et Vilnet

Lophozia obtusa $=$ Obtusifolium obtusum (Lindb.) S. W. Arnell

Lophozia sudetica $=$ Barbilophozia sudetica (Nees ex Huebener) L. Söderstr., De Roo et Hedd.

Lophozia ventricosa $=$ Lophozia ventricosa (Dicks.) Dumort.

Lophozia wenzelii = Lophozia wenzelii (Nees) Steph.

Lunularia cruciata $=$ Lunularia cruciata (L.) Dumort. ex Lindb.

Mannia fragrans = Mannia fragrans (Balb.) Frye et L. Clark

Mannia triandra = Mannia triandra (Scop.) Grolle

Marchantia polypmorpha subsp. polymorpha = Marchantia polymorpha L. subsp. polymorpha

Marchantia polypmorpha subsp. ruderalis = Marchantia polymorpha L. subsp. ruderalis Bischl. et

Boissel.-Dub.

Marsupella emarginata = Marsupella emarginata (Ehrh.) Dumort.

Marsupella funckii $=$ Marsupella funckii (F. Weber et D. Mohr) Dumort.

Marsupella sprucei $=$ Marsupella sprucei (Limpr.) Bernet

Metzgeria conjugata subsp. conjugata $=$ Metzgeria conjugata $\mathrm{Lindb}$.

Metzgeria furcata $=$ Metzgeria furcata (L.) Corda

Nardia geoscyphus = Nardia geoscyphus (De Not.) Lindb.

Nardia scalaris $=$ Nardia scalaris Gray

Nowellia curvifolia $=$ Nowellia curvifolia (Dicks.) Mitt.

Oxymitra incrassata $=$ Oxymitra incrassata (Brot.) Sérgio et Sim-Sim

Pedinophyllum interruptum $=$ Pedinophyllum interruptum (Nees) Kaal.

Pellia endiviifolia = Apopellia endiviifolia (Dicks.) Nebel et D. Quandt

Pellia epiphylla = Pellia epiphylla (L.) Corda

Plagiochila asplenioides = Plagiochila asplenioides (L.) Dumort.

Plagiochila porelloides = Plagiochila porelloides (Torr. ex Nees) Lindenb.

Porella arboris-vitae $=$ Porella arboris-vitae $($ With.) Grolle

Porella baueri $=$ Porella baueri (Schiffn.) C. E. O. Jensen

Porella cordaeana $=$ Porella cordaeana (Huebener) Moore

Porella platyphylla $=$ Porella platyphylla (L.) Pfeiff.

Preissia quadrata $=$ Marchantia quadrata Scop.

Ptilidium pulcherrimum = Ptilidium pulcherrimum (Weber) Vain.

Radula complanata $=$ Radula complanata (L.) Dumort.

Radula lindenbergiana $=$ Radula lindenbergiana Gottsche ex C. Hartm.

Reboulia hemisphaerica $=$ Reboulia hemisphaerica $(\mathrm{L}$.) Raddi

Riccardia chamedryfolia = Riccardia chamedryfolia (With.) Grolle

Riccardia incurvata $=$ Riccardia incurvata Lindb.

Riccardia latifrons $=$ Riccardia latifrons (Lindb.) Lindb.

Riccardia multifida = Riccardia multifida (L.) Gray

Riccardia palmata $=$ Riccardia palmata (Hedw.) Carruth.

Riccia bifurca = Riccia bifurca Hoffm.

Riccia canaliculata = Riccia canaliculata Hoffm.

Riccia cavernosa = Riccia cavernosa Hoffm.

Riccia ciliata = Riccia ciliata Hoffm. 
Riccia ciliifera $=$ Riccia ciliifera Link

Riccia crozalsii = Riccia crozalsii Levier

Riccia fluitans = Riccia fluitans L.

Riccia frostii = Riccia frostii Austin

Riccia glauca $=$ Riccia glauca $\mathrm{L}$.

Riccia gougetiana $=$ Riccia gougetiana Durieu et Mont.

Riccia buebeneriana $=$ Riccia buebeneriana Lindenb.

Riccia papillosa $=$ Riccia papillos $a$ Moris

Riccia rhenana $=$ Riccia rbenana Lorb. ex Müll. Frib.

Riccia sorocarpa $=$ Riccia sorocarpa Bisch.

Riccia subbifurca = Riccia subbifurca Warnst. ex Croz.

Riccia warnstorfi $=$ Riccia warnstorfi $i$ Limpr. ex Warnst.

Ricciocarpos natans $=$ Ricciocarpos natans $(\mathrm{L}$.) Corda

Scapania aequiloba $=$ Scapania aequiloba (Schwägr.) Dumort.

Scapania apiculata $=$ Scapania apiculata Spruce

Scapania aspera $=$ Scapania aspera M. Bernet et Bernet

Scapania calcicola = Scapania calcicola (Arnell et J. Perss.) Ingham

Scapania curta = Scapania curta (Mart.) Dumort.

Scapania irrigua $=$ Scapania irrigua Nees

Scapania lingulata = Scapania lingulata $\mathrm{H}$. Buch

Scapania mucronata = Scapania mucronata $\mathrm{H}$. Buch

Scapania nemorea = Scapania nemorea (L.) Grolle

Scapania scandica = Scapania scandica (Arnell et H. Buch) Macvicar

Scapania umbrosa = Scapania umbrosa (Schrad.) Dumort.

Scapania undulata = Scapania undulata (L.) Dumort.

Sphaerocarpos texanus auct. $=$ Sphaerocarpos europaeus Lorb.

Trichocolea tomentella $=$ Trichocolea tomentella (Ehrh.) Dumort.

Tritomaria exsecta = Tritomaria exsecta (Schmidel) Schiffn. ex Loeske

Tritomaria exsectiformis = Tritomaria exsectiformis (Breidl.) Schiffn. ex Loeske

Tritomaria quinquedentata $=$ Trilophozia quinquedentata (Huds.) Bakalin

\section{Mosses}

Acaulon muticum = Acaulon muticum (Hedw.) Müll. Hal.

Acaulon triquetrum = Acaulon triquetrum (Spruce) Müll. Hal.

Aloina aloides $=$ Aloina aloides (Koch ex Schultz) Kindb.

Aloina ambigua = Aloina ambigua (Bruch et Schimp.) Limpr.

Aloina rigida $=$ Aloina rigida (Hedw.) Limpr.

Amblyodon dealbatus $=$ Amblyodon dealbatus (Hedw.) P. Beauv.

Amblystegium confervoides $=$ Serpoleskea confervoides (Brid.) Schimp.

Amblystegium fluviatile $=$ Hygroamblystegium fluviatile (Hedw.) Loeske

Amblystegium humile $=$ Hygroamblystegium humile (P. Beauv.) Vanderp., Goffinet et Hedenäs

Amblystegium radicale $=$ Pseudocampylium radicale (P. Beauv.) Vanderp. et Hedenäs

Amblystegium serpens $=$ Amblystegium serpens (Hedw.) Schimp.

Amblystegium subtile = Pseudoamblystegium subtile (Hedw.) Vanderp. et Hedenäs

Amblystegium tenax $=$ Hygroamblystegium tenax (Hedw.) Jenn.

Amblystegium varium $=$ Hygroamblystegium varium (Hedw.) Mönk.

Amphidium mougeotii = Amphidium mougeotii (Schimp.) Schimp. 
Anacamptodon splachnoides $=$ Anacamptodon splachnoides (Froel. ex Brid.) Brid . Andreaea rupestris $=$ Andreaea rupestris $\mathrm{Hedw}$. Anomodon attenuatus $=$ Pseudanomodon attenuatus (Hedw.) Ignatov et Fedosov Anomodon longifolius = Anomodon longifolius (Schleich. ex Brid.) Hartm. Anomodon rostratus $=$ Claopodium rostratum (Hedw.) Ignatov Anomodon rugelii $=$ Anomodon rugelii (Müll. Hal.) Keissl. Anomodon viticulosus = Anomodon viticulosus (Hedw.) Hook. et Taylor Antitrichia curtipendula = Antitrichia curtipendula (Hedw.) Brid. Aphanorrhegma patens $=$ Physcomitrium patens $(\mathrm{Hedw}$.$) Mitt.$ Archidium alternifolium $=$ Archidium alternifolium (Hedw.) Mitt. Atrichum angustatum = Atrichum angustatum (Brid.) Bruch et Schimp. Atrichum undulatum $=$ Atrichum undulatum (Hedw.) P. Beauv. Aulacomnium androgynum = Aulacomnium androgynum (Hedw.) Schwägr. Aulacomnium palustre = Aulacomnium palustre (Hedw.) Schwägr. Barbula convoluta $=$ Streblotrichum convolutum (Hedw.) P. Beauv. Barbula indica $=$ Hydrogonium consanguineum (Thwaites et Mitt.) Hilp. Barbula unguiculata $=$ Barbula unguiculata Hedw. Bartramia halleriana $=$ Bartramia halleriana Hedw. Bartramia ithyphylla = Bartramia ithyphylla Brid. Bartramia pomiformis = Bartramia pomiformis Hedw. Blindia acuta $=$ Blindia acuta (Hedw.) Bruch et Schimp. Brachydontium trichodes = Brachydontium trichodes $(\mathrm{F}$. Weber) Milde Brachythecium albicans $=$ Brachythecium albicans (Hedw.) Schimp. Brachythecium campestre = Brachythecium campestre (Müll. Hal.) Schimp. Brachythecium capillaceum = Brachythecium capillaceum (F. Weber et D. Mohr) Giacom. Brachythecium geheebii = Brachythecium geheebii Milde Brachythecium glareosum = Brachythecium glareosum (Bruch ex Spruce) Schimp. Brachythecium laetum = Brachythecium laetum (Brid.) Schimp. Brachythecium mildeanum = Brachythecium mildeanum (Schimp.) Schimp. Brachythecium plumosum = Sciuro-hypnum plumosum (Hedw.) Ignatov et Huttunen Brachythecium populeum = Sciuro-hypnum populeum (Hedw.) Ignatov et Huttunen Brachythecium reflexum = Sciuro-hypnum reflexum (Starke) Ignatov et Huttunen Brachythecium rivulare $=$ Brachythecium rivulare Schimp. Brachythecium rutabulum = Brachythecium rutabulum (Hedw.) Schimp. Brachythecium salebrosum = Brachythecium salebrosum (Hoffm. ex F. Weber et D. Mohr) Schimp. Brachythecium velutinum $=$ Brachytheciastrum velutinum (Hedw.) Ignatov et Huttunen Bryoerythrophyllum recurvirostrum $=$ Bryoerythrophyllum recurvirostrum (Hedw.) P. C. Chen Bryum algovicum $=$ Ptychostomum compactum Hornsch.

Bryum alpinum = Imbribryum alpinum (Huds. ex With.) N. Pedersen Bryum argenteum $=$ Bryum argenteum $\mathrm{Hedw}$.

Bryum bicolor $=$ Bryum bicolor Dicks.

Bryum caespiticium = Ptychostomum imbricatulum (Müll. Hal.) Holyoak et N. Pedersen Bryum capillare $=$ Ptychostomum capillare (Hedw.) Holyoak et N. Pedersen Bryum creberrimum = Ptychostomum creberrimum (Taylor) J. R. Spence et H. P. Ramsay Bryum elegans $=$ Ptychostomum elegans (Nees in Brid.) D. Bell et Holyoak Bryum funckii $=$ Ptychostomum funkii (Schwägr.) J. R. Spence Bryum gemmiferum $=$ Bryum gemmiferum $\mathrm{R}$. Wilczek et Demaret Bryum gemmilucens $=$ Bryum gemmilucens $\mathrm{R}$. Wilczek et Demaret 
Bryum imbricatum = Ptychostomum inclinatum (Sw. ex Brid.) J. R. Spence

Bryum intermedium $=$ Ptychostomum intermedium (Brid.) J. R. Spence

Bryum klinggraeffi $=$ Bryum klinggraeffi Schimp.

Bryum mildeanum = Imbribryum mildeanum (Jur.) J. R. Spence

Bryum moravicum = Ptychostomum moravicum (Podp.) Ros et Mazimpaka

Bryum pallens $=$ Ptychostomum pallens (Sw. ex anon.) J. R. Spence

Bryum pallescens $=$ Ptychostomum pallescens (Schleich. ex Schwägr.) J. R. Spence

Bryum pseudotriquetrum = Ptychostomum pseudotriquetrum (Hedw.) J. R. Spence et H. P. Ramsay ex Holyoak et N. Pedersen

Bryum radiculosum $=$ Bryum radiculosum Brid.

Bryum rubens $=$ Ptychostomum rubens (Mitt.) Holyoak et N. Pedersen

Bryum ruderale $=$ Bryum ruderale Crundw. et Nyholm

Bryum stirtonii $=$ Bryum stirtonii Schimp.

Bryum torquescens $=$ Ptychostomum torquescens (Bruch et Schimp.) Ros et Mazimpaka

Bryum turbinatum = Ptychostomum turbinatum (Hedw.) J. R. Spence

Bryum uliginosum = Ptychostomum cernuum (Hedw.) Hornsch.

Bryum violaceum $=$ Bryum violaceum Crundw. et Nyholm

Bryum warneum $=$ Ptychostomum warneum (Röhl.) J. R. Spence

Bryum weigelii = Ptychostomum weigelii (Biehler) J. R. Spence

Buxbaumia aphylla = Buxbaumia aphylla Hedw.

Buxbaumia viridis = Buxbaumia viridis (Moug. ex Lam. et DC.) Brid. ex Moug. et Nestl.

Calliergon cordifolium $=$ Calliergon cordifolium (Hedw.) Kindb.

Calliergon giganteum $=$ Calliergon giganteum (Schimp.) Kindb.

Calliergon stramineum $=$ Straminergon stramineum (Dicks. ex Brid.) Hedenäs

Calliergonella cuspidata $=$ Calliergonella cuspidata $($ Hedw.) Loeske

Campylium calcareum = Campylophyllopsis calcarea (Crundw. et Nyholm) Ochyra

Campylium chrysophyllum $=$ Campyliadelphus chrysophyllus (Brid.) R. S. Chopra

Campylium elodes $=$ Campyliadelphus elodes (Lindb.) Kanda

Campylium polygamum $=$ Drepanocladus polygamus (Schimp.) Hedenäs

Campylium stellatum $=$ Campylium stellatum (Hedw.) Lange et C. E. O. Jensen

Campylopus introflexus $=$ Campylopus introflexus (Hedw.) Brid.

Campylopus pyriformis $=$ Campylopus pyriformis (Schultz) Brid.

Campylostelium saxicola $=$ Campylostelium saxicola (F. Weber et D. Mohr) Bruch et Schimp.

Ceratodon purpureus $=$ Ceratodon purpureus (Hedw.) Brid.

Cinclidotus danubicus = Cinclidotus danubicus Schiffn. et Baumgartner

Cinclidotus fontinaloides $=$ Cinclidotus fontinaloides (Hedw.) P. Beauv.

Cinclidotus riparius $=$ Cinclidotus riparius (Host ex Brid.) Arn.

Cirriphyllum piliferum $=$ Cirriphyllum piliferum (Hedw.) Grout

Cirriphyllum tommasinii = Brachythecium tommasinii (Sendtn. ex Boulay) Ignatov et Huttunen

Climacium dendroides $=$ Climacium dendroides (Hedw.) F. Weber et D. Mohr

Cnestrum schisti $=$ Cnestrum schisti (F. Weber et D. Mohr) I. Hagen

Conardia compacta = Conardia compacta (Drumm. ex Müll. Hal.) H. Rob.

Coscinodon cribrosus $=$ Coscinodon cribrosus (Hedw.) Spruce

Cratoneuron filicinum $=$ Cratoneuron filicinum $(\mathrm{Hedw}$.) Spruce

Crossidium crassinerve $=$ Crossidium crassinervium (De Not.) Jur.

Crossidium laxefilamentosum $=$ Crossidium laxefilamentosum W. Frey et Kürschner

Ctenidium molluscum = Ctenidium molluscum (Hedw.) Mitt.

Cynodontium polycarpon p. p. = Cynodontium polycarpon (Hedw.) Schimp. 
Cynodontium tenellum $=$ Cynodontium tenellum (Schimp.) Limpr.

Desmatodon cernuus $=$ Tortula cernua $($ Huebener $)$ Lindb.

Desmatodon heimii $=$ Henediella heimii (Hedw.) R. H. Zander

Dichodontium pellucidum = Dichodontium pellucidum (Hedw.) Schimp.

Dicranella cerviculata $=$ Dicranella cerviculata (Hedw.) Schimp.

Dicranella crispa = Dicranella crispa (Hedw.) Schimp.

Dicranella heteromalla = Dicranella beteromalla (Hedw.) Schimp.

Dicranella howei $=$ Dicranella howei Renauld et Cardot

Dicranella bumilis = Dicranella bumilis $\mathrm{R}$. Ruthe

Dicranella rufescens $=$ Dicranella rufescens (Dicks.) Schimp.

Dicranella schreberiana $=$ Dicranella schreberiana $(\mathrm{Hedw}$.$) Dixon$

Dicranella staphylina $=$ Dicranella staphylina $\mathrm{H}$. Whitehouse

Dicranella subulata = Dicranella subulata (Hedw.) Schimp.

Dicranella varia $=$ Dicranella varia (Hedw.) Schimp.

Dicranodontium denudatum $=$ Dicranodontium denudatum (Brid.) E. Britton

Dicranoweisia cirrata $=$ Dicranoweisia cirrata (Hedw.) Lindb.

Dicranum bonjeanii $=$ Dicranum bonjeanii De Not.

Dicranum flagellare $=$ Dicranum flagellare Hedw.

Dicranum fulvum = Dicranum fulvum Hook.

Dicranum montanum $=$ Dicranum montanum Hedw.

Dicranum muehlenbeckii $=$ Dicranum mueblenbeckii Bruch et Schimp.

Dicranum polysetum = Dicranum polysetum Sw. ex anon.

Dicranum scoparium = Dicranum scoparium Hedw.

Dicranum spurium $=$ Dicranum spurium Hedw.

Dicranum tauricum $=$ Dicranum tauricum Sapjegin

Dicranum viride $=$ Dicranum viride (Sull. et Lesq.) Lindb.

Didymodon acutus $=$ Didymodon acutus (Brid.) K. Saito

Didymodon cordatus = Didymodon cordatus Jur.

Didymodon fallax $=$ Didymodon fallax (Hedw.) R. H. Zander

Didymodon ferrugineus = Didymodon ferrugineus (Schimp. ex Besch.) M. O. Hill

Didymodon glaucus $=$ Didymodon glaucus Ryan

Didymodon insulanus = Didymodon insulanus (De Not.) M. O. Hill

Didymodon luridus $=$ Didymodon luridus Hornsch.

Didymodon rigidulus $=$ Didymodon rigidulus $\mathrm{Hedw}$.

Didymodon sinuosus $=$ Didymodon sinuosus (Mitt.) Delogne

Didymodon spadiceus $=$ Didymodon spadiceus (Mitt.) Limpr.

Didymodon tophaceus = Didymodon tophaceus (Brid.) Lisa subsp. tophaceus

Didymodon vinealis $=$ Didymodon vinealis (Brid.) R. $\mathrm{H}$. Zander

Diphyscium foliosum = Diphyscium foliosum (Hedw.) D. Mohr

Distichium capillaceum $=$ Distichium capillaceum (Hedw.) Bruch et Schimp.

Ditrichum crispatissimum $=$ Flexitrichum gracile (Mitt.) Ignatov et Fedosov

Ditrichum cylindricum $=$ Trichodon cylindricus (Hedw.) Schimp.

Ditrichum flexicaule $=$ Flexitrichum flexicaule (Schwägr.) Ignatov et Fedosov

Ditrichum heteromallum $=$ Ditrichum heteromallum (Hedw.) E. Britton

Ditrichum pallidum = Ditrichum pallidum (Hedw.) Hampe

Ditrichum pusillum = Ditrichum pusillum (Hedw.) Hampe

Drepanocladus aduncus = Drepanocladus aduncus (Hedw.) Warnst.

Drepanocladus cossonii $=$ Scorpidium cossonii (Schimp.) Hedenäs 
Drepanocladus lycopodioides $=$ Drepanocladus lycopodioides (Brid.) Warnst.

Drepanocladus sendtneri $=$ Drepanocladus sendtneri (Schimp. ex H. Müll.) Warnst.

Drepanocladus sordidus = Drepanocladus sordidus (Müll. Hal.) Hedenäs

Encalypta ciliata $=$ Encalypta ciliata Hedw.

Encalypta streptocarpa $=$ Encalypta streptocarpa $\mathrm{Hedw}$.

Encalypta vulgaris = Encalypta vulgaris $\mathrm{Hedw}$.

Entodon concinnus $=$ Entodon concinnus (De Not.) Paris

Entosthodon fascicularis = Entosthodon fascicularis (Hedw.) Müll. Hal.

Entosthodon hungaricus $=$ Entosthodon hungaricus (Boros) Loeske

Ephemerum cohaerens $=$ Ephemerum cohaerens (Hedw.) Hampe

Ephemerum minutissimum = Ephemerum serratum (Hedw.) Hampe

Ephemerum recurvifolium $=$ Ephemerum recurvifolium (Dicks.) Boulay

Ephemerum serratum auct. non (Hedw.) Hampe = Ephemerum stoloniferum (Hedw.) L. T. Ellis et M. J. Price

Ephemerum sessile = Ephemerum crassinervium (Schwägr.) Hampe subsp. sessile (Bruch) Holyoak Eucladium verticillatum $=$ Eucladium verticillatum (With.) Bruch et Schimp.

Eurhynchium angustirete $=$ Eurhynchium angustirete (Broth.) T. J. Kop.

Eurhynchium crassinervium $=$ Cirriphyllum crassinervium (Taylor) Loeske et M. Fleisch.

Eurbynchium flotowianum $=$ Sciuro-hypnum flotowianum (Sendtn.) Ignatov et Huttunen

Eurhynchium hians $=$ Oxyrrhynchium hians (Hedw.) Loeske

Eurhynchium praelongum $=$ Kindbergia praelonga $($ Hedw.) Ochyra

Eurbynchium pulchellum = Eurbynchiastrum pulchellum (Hedw.) Ignatov et Huttunen

Eurbynchium pumilum = Microeurhynchium pumilum (Wilson) Ignatov et Vanderp.

Eurhynchium schleicheri $=$ Oxyrrbynchium schleicheri (R. Hedw.) Röll

Eurhynchium speciosum $=$ Oxyrrhynchium speciosum (Brid.) Warnst.

Eurbynchium striatulum = Plasteurhynchium striatulum (Spruce) M. Fleisch.

Eurhynchium striatum = Eurhynchium striatum (Hedw.) Schimp.

Fabronia ciliaris $=$ Fabronia ciliaris (Brid.) Brid.

Fabronia pusilla $=$ Fabronia pusilla Raddi

Fissidens adianthoides $=$ Fissidens adianthoides Hedw.

Fissidens arnold $i i=$ Fissidens arnoldii $\mathrm{R}$. Ruthe

Fissidens bryoides $=$ Fissidens bryoides $\mathrm{Hedw}$.

Fissidens crassipes subsp. crassipes $=$ Fissidens crassipes Wilson ex Bruch et Schimp.

Fissidens curvatus $=$ Fissidens curvatus Hornsch.

Fissidens dubius p. p. = Fissidens dubius P. Beauv. var. dubius

Fissidens exilis = Fissidens exilis Hedw.

Fissidens gracilifolius $=$ Fissidens gracilifolius Brugg.-Nann. et Nyholm

Fissidens gymnandrus = Fissidens gymnandrus Buse

Fissidens incurvus $=$ Fissidens incurvus Starke ex Röhl.

Fissidens pusillus $=$ Fissidens pusillus (Wilson) Milde

Fissidens taxifolius $=$ Fissidens taxifolius $\mathrm{Hedw}$.

Fissidens viridulus $=$ Fissidens viridulus $(\mathrm{Sw}$.$) Wahlenb.$

Fontinalis antipyretica $=$ Fontinalis antipyretica Hedw.

Fontinalis hypnoides $=$ Fontinalis hypnoides $\mathrm{C}$. Hartm.

Funaria hygrometrica $=$ Funaria hygrometrica Hedw.

Funaria mublenbergii = Entosthodon mublenbergii (Turner) Fife

Funaria pulchella $=$ Entosthodon pulchellus $(\mathrm{H}$. Philib.) Brugués

Grimmia anodon = Grimmia anodon Bruch et Schimp. 
Grimmia crinita $=$ Grimmia crinita Brid.

Grimmia decipiens $=$ Grimmia decipiens (Schultz) Lindb.

Grimmia dissimulata $=$ Grimmia dissimulata E. Maier

Grimmia elatior $=$ Grimmia elatior Bruch ex Bals.-Criv. et De Not.

Grimmia funalis = Grimmia funalis (Schwägr.) Bruch et Schimp.

Grimmia hartmanii = Grimmia hartmanii Schimp.

Grimmia laevigata $=$ Grimmia laevigata (Brid.) Brid.

Grimmia lisae = Grimmia lisae De Not.

Grimmia longirostris $=$ Grimmia longirostris Hook.

Grimmia montana = Grimmia montana Bruch et Schimp.

Grimmia mueblenbeckii = Grimmia mueblenbeckii Schimp.

Grimmia orbicularis = Grimmia orbicularis Bruch ex Wilson

Grimmia ovalis = Grimmia ovalis (Hedw.) Lindb.

Grimmia plagiopodia $=$ Grimmia plagiopodia $\mathrm{Hedw}$.

Grimmia pulvinata $=$ Grimmia pulvinata (Hedw.) Sm.

Grimmia teretinervis $=$ Grimmia teretinervis Limpr.

Grimmia tergestina $=$ Grimmia tergestina Tomm. ex Bruch et Schimp.

Grimmia trichophylla $=$ Grimmia trichophylla Grev.

Gymnostomum aeruginosum = Gymnostomum aeruginosum $\mathrm{Sm}$.

Gymnostomum calcareum $=$ Gymnostomum calcareum Nees et Hornsch.

Gymnostomum viridulum = Gymnostomum viridulum Brid.

Gyroweisia tenuis $=$ Gyroweisia tenuis (Hedw.) Schimp.

Hamatocaulis vernicosus $=$ Hamatocaulis vernicosus (Mitt.) Hedenäs

Hedwigia ciliata p. p. = Hedwigia ciliata (Hedw.) P. Beauv.

Hedwigia stellata $=$ Hedwigia stellata Hedenäs

Helodium blandowii $=$ Helodium blandowii (F. Weber et D. Mohr) Warnst.

Herzogiella seligeri $=$ Herzogiella seligeri (Brid.) Z. Iwats.

Heterocladium dimorphum $=$ Heterocladiella dimorpha (Brid.) Ignatov et Fedosov

Hilpertia velenovskyi $=$ Hilpertia velenovskyi (Schiffn.) R. $\mathrm{H}$. Zander

Homalia besseri = Alleniella besseri (Lobarz.) S. Olsson, Enroth et D. Quandt

Homalia trichomanoides $=$ Homalia trichomanoides (Hedw.) Brid.

Homalothecium lutescens $=$ Homalothecium lutescens $($ Hedw.) H. Rob.

Homalothecium philippeanum $=$ Homalothecium philippeanum (Spruce) Schimp.

Homalothecium sericeum $=$ Homalothecium sericeum (Hedw.) Schimp.

Homomallium incurvatum = Homomallium incurvatum (Schrad. ex Brid.) Loeske

Hygrohypnum luridum = Hygrohypnum luridum (Hedw.) Jenn.

Hylocomium brevirostre $=$ Loeskeobryum brevirostre (Brid.) M. Fleisch.

Hylocomium splendens $=$ Hylocomium splendens (Hedw.) Schimp.

Hymenostylium recurvirostrum $=$ Hymenostylium recurvirostrum (Hedw.) Dixon

Hypnum cupressiforme $=$ Hypnum cupressiforme Hedw.

Hypnum jutlandicum = Hypnum jutlandicum Holmen et E. Warncke

Hypnum lindbergii $=$ Calliergonella lindbergii (Mitt.) Hedenäs

Hypnum pallescens $=$ Hypnum pallescens (Hedw.) P. Beauv. var. reptile (Michx.) Husn.

Hypnum vaucheri $=$ Buckia vaucheri (Lesq.) D. Rios, M. T. Gallego et J. Guerra

Isopterygiopsis pulchella $=$ Isopterygiopsis pulchella (Hedw.) Z. Iwats.

Isothecium alopecuroides = Isothecium alopecuroides (Lam. ex Dubois) Isov.

Isothecium myosuroides = Isothecium myosuroides Brid.

Leptobryum pyriforme = Leptobryum pyriforme (Hedw.) Wilson 
Leptodictyum riparium $=$ Leptodictyum riparium (Hedw.) Warnst.

Leptodon smithii $=$ Leptodon smithii (Hedw.) F. Weber et D. Mohr

Leskea polycarpa $=$ Leskea polycarpa $\mathrm{Hedw}$.

Leucobryum glaucum = Leucobryum glaucum (Hedw.) Ångstr.

Leucobryum juniperoideum = Leucobryum juniperoideum (Brid.) Müll. Hal.

Leucodon sciuroides = Leucodon sciuroides (Hedw.) Schwägr.

Meesia triquetra = Meesia triquetra (L. ex Jolycl.) Ångstr.

Mnium hornum $=$ Mnium hornum Hedw.

Mnium lyocopodioides = Mnium lycopodioides Schwägr.

Mnium marginatum = Mnium marginatum (Dicks.) P. Beauv.

Mnium spinulosum $=$ Mnium spinulosum Bruch et Schimp.

Mnium stellare $=$ Mnium stellare Hedw.

Mnium thomsonii = Mnium thomsonii Schimp.

Myurella julacea $=$ Myurella julacea (Schwägr.) Schimp.

Neckera complanata $=$ Alleniella complanata (Hedw.) S. Olsson, Enroth et D. Quandt

Neckera crispa $=$ Exsertotheca crispa (Hedw.) S. Olsson, Enroth et D. Quandt

Neckera pennata $=$ Neckera pennata Hedw.

Neckera pumila $=$ Neckera pumila Hedw.

Orthothecium intricatum $=$ Orthothecium intricatum (Hartm.) Schimp.

Orthotrichum affine $=$ Lewinskya affinis (Schrad. ex Brid.) F. Lara, Garilleti et Goffinet

Orthotrichum anomalum = Orthotrichum anomalum Hedw.

Orthotrichum cupulatum $=$ Orthotrichum cupulatum Brid.

Orthotrichum diaphanum $=$ Orthotrichum diaphanum Brid.

Orthotrichum gymnostomum $=$ Nybolmiella gymnostoma (Bruch ex Brid.) Holmen et E. Warncke

Orthotrichum lyellii = Pulvigera lyellii (Hook. et Taylor) Plášek, Sawicki et Ochyra

Orthotrichum obtusifolium $=$ Nybolmiella obtusifolia (Brid.) Holmen et E. Warncke

Orthotrichum pallens $=$ Orthotrichum pallens Bruch ex Brid.

Orthotrichum patens $=$ Orthotrichum patens Bruch ex Brid.

Orthotrichum pumilum p. p. = Orthotrichum pumilum Sw. ex anon.

Orthotrichum roger $=$ Orthotrichum rogeri Brid.

Orthotrichum rupestre = Lewinskya rupestris (Schleich. ex Schwägr.) F. Lara, Garilleti et Goffinet

Orthotrichum speciosum = Lewinskya speciosa (Nees) F. Lara, Garilleti et Goffinet

Orthotrichum sprucei = Orthotrichum sprucei Mont.

Orthotrichum stramineum = Orthotrichum stramineum Hornsch. ex Brid.

Orthotrichum striatum = Lewinskya striata (Hedw.) F. Lara, Garilleti et Goffinet

Orthotrichum urnigerum $=$ Orthotrichum urnigerum Myrin

Oxystegus tenuirostris = Chionoloma tenuirostre (Hook. et Taylor) M. Alonso, M. J. Cano et J. A.

Jiménez

Palustriella commutata p. p. = Palustriella commutata (Hedw.) Ochyra

Paraleucobryum longifolium $=$ Paraleucobryum longifolium (Hedw.) Loeske

Phascum curvicolle $=$ Microbryum curvicollum (Hedw.) R. H. Zander

Phascum cuspidatum = Tortula acaulon (With.) R. H. Zander

Phascum floerkeanum = Microbryum floerkeanum (F. Weber et D. Mohr) Schimp.

Phascum leptophyllum = Chenia leptophylla (Müll. Hal.) R. H. Zander

Philonotis arnellii $=$ Philonotis capillaris Lindb.

Philonotis caespitosa $=$ Philonotis caespitosa Jur.

Philonotis calcarea $=$ Philonotis calcarea (Bruch et Schimp.) Schimp.

Philonotis fontana $=$ Philonotis fontana (Hedw.) Brid. 
Philonotis marchica $=$ Philonotis marchica (Hedw.) Brid.

Physcomitrium eurystomum $=$ Physcomitrium eurystomum Sendtn.

Physcomitrium pyriforme $=$ Physcomitrium pyriforme $($ Hedw.) Bruch et Schimp.

Physcomitrium sphaericum = Physcomitrium sphaericum (C. F.Ludw. ex Schkuhr) Brid.

Plagiobryum zierii = Ptychostomum zieri (Hedw.) Holyoak et N. Pedersen

Plagiomnium affine = Plagiomnium affine (Blandow ex Funck) T. J. Kop.

Plagiomnium cuspidatum = Plagiomnium cuspidatum (Hedw.) T. J. Kop.

Plagiomnium elatum = Plagiomnium elatum (Bruch et Schimp.) T. J. Kop.

Plagiomnium ellipticum = Plagiomnium ellipticum (Brid.) T. J. Kop.

Plagiomnium medium = Plagiomnium medium (Bruch et Schimp.) T. J. Kop.

Plagiomnium rostratum $=$ Plagiomnium rostratum (Schrad.) T. J. Kop.

Plagiomnium undulatum = Plagiomnium undulatum (Hedw.) T. J. Kop.

Plagiopus oederianus = Plagiopus oederianus (Sw.) H. A. Crum et L. E. Anderson

Plagiothecium cavifolium $=$ Plagiothecium cavifolium (Brid.) Z. Iwats.

Plagiothecium curvifolium = Plagiothecium curvifolium Schlieph. ex Limpr.

Plagiothecium denticulatum = Plagiothecium denticulatum (Hedw.) Schimp. var. denticulatum

Plagiothecium laetum = Plagiothecium laetum Schimp.

Plagiothecium nemorale $=$ Plagiothecium nemorale $($ Mitt.) A. Jaeger

Plagiothecium platyphyllum = Plagiothecium platyphyllum Mönk.

Plagiothecium ruthei $=$ Plagiothecium denticulatum (Hedw.) Schimp. var. undulatum R. Ruthe ex Geh.

Plagiothecium succulentum = Plagiothecium succulentum (Wilson) Lindb.

Plagiothecium undulatum = Plagiothecium undulatum (Hedw.) Schimp.

Platydictya jungermannioides $=$ Platydictya jungermannioides $($ Brid.) H. A. Crum

Platygyrium repens $=$ Platygyrium repens (Brid.) Schimp.

Platyhypnidium riparioides $=$ Rhynchostegium riparioides (Hedw.) Cardot

Pleuridium acuminatum $=$ Pleuridium acuminatum Lindb.

Pleuridium subulatum = Pleuridium subulatum (Hedw.) Rabenh.

Pleurochaete squarrosa $=$ Tortella squarrosa (Brid.) Limpr.

Pleurozium schreberi = Pleurozium schreberi (Willd. ex Brid.) Mitt.

Pogonatum aloides $=$ Pogonatum aloides (Hedw.) P. Beauv.

Pogonatum nanum = Pogonatum nanum (Hedw.) P. Beauv.

Pogonatum urnigerum = Pogonatum urnigerum (Hedw.) P. Beauv.

Poblia andalusica $=$ Poblia andalusica (Höhn.) Broth .

Poblia annotina $=$ Poblia annotina (Hedw.) Lindb.

Poblia camptotrachela $=$ Poblia camptotrachela $($ Renauld et Cardot) Broth.

Pohlia cruda = Poblia cruda (Hedw.) Lindb.

Poblia elongata $=$ Poblia elongata Hedw.

Poblia lescuriana = Poblia lescuriana (Sull.) Ochi

Poblia lutescens $=$ Poblia lutescens (Limpr.) H. Lindb.

Poblia melanodon = Poblia melanodon (Brid.) A. J. Shaw

Poblia nutans $=$ Poblia nutans (Hedw.) Lindb. subsp. nutans

Pohlia proligera $=$ Poblia proligera $($ Kindb.) Lindb. ex Broth.

Pohlia schimperi $=$ Pohlia nutans (Hedw.) Lindb. subsp. schimperi (Müll. Hal.) Nyholm

Pohlia wahlenbergii = Poblia wablenbergii (F. Weber et D. Mohr) A. L. Andrews

Polytrichum alpinum $=$ Polytrichastrum alpinum (Hedw.) G. L. Sm.

Polytrichum commune p. p. = Polytrichum commune Hedw.

Polytrichum formosum = Polytrichum formosum Hedw. 
Polytrichum juniperinum $=$ Polytrichum juniperinum Hedw.

Polytrichum longisetum = Polytrichum longisetum Sw. ex Brid.

Polytrichum piliferum $=$ Polytrichum piliferum $\mathrm{Hedw}$.

Polytrichum strictum = Polytrichum strictum Menzies ex Brid.

Pottia bryoides $=$ Tortula protobryoides $\mathrm{R}$. H. Zander

Pottia davalliana $=$ Microbryum davallianum $(\mathrm{Sm}$.$) R. H. Zander$

Pottia intermedia $=$ Tortula caucasica Broth.

Pottia lanceolata $=$ Tortula lindbergii Broth.

Pottia mutica $=$ Microbryum muticum (Venturi) Cl. Schneid., Th. Schneid. et Mahévas

Pottia starckeana = Microbryum starckeanum (Hedw.) R. H. Zander

Pottia truncata $=$ Tortula truncata (Hedw.) Mitt.

Pseudephemerum nitidum = Pseudephemerum nitidum (Hedw.) Loeske

Pseudocrossidium hornschuchianum $=$ Pseudocrossidium hornschuchianum (Schultz) R. H. Zander

Pseudocrossidium revolutum $=$ Pseudocrossidium revolutum (Brid.) R. H. Zander

Pseudoleskea saviana = Lescuraea saviana (De Not.) E. Lawton

Pseudoleskeella catenulata $=$ Pseudoleskeella catenulata (Brid. ex Schrad.) Kindb.

Pseudoleskeella nervosa $=$ Pseudoleskeella nervosa (Brid.) Nyholm

Pseudotaxiphyllum elegans = Pseudotaxiphyllum elegans (Brid.) Z. Iwats.

Pterigynandrum filiforme $=$ Pterigynandrum filiforme Hedw.

Pterogonium gracile $=$ Nogopterium gracile $($ Hedw.) Crosby et W. R. Buck

Pterygoneurum lamellatum = Pterygoneurum lamellatum (Lindb.) Jur.

Pterygoneurum ovatum = Pterygoneurum ovatum (Hedw.) Dixon

Pterygoneurum subsessile $=$ Pterygoneurum subsessile (Brid.) Jur.

Ptilium crista-castrensis $=$ Ptilium crista-castrensis $($ Hedw.) De Not.

Pylaisia polyantha $=$ Pylaisia polyantha (Hedw.) Schimp.

Pyramidula tetragona = Pyramidula tetragona (Brid.) Brid.

Racomitrium aciculare $=$ Racomitrium aciculare (Hedw.) Brid.

Racomitrium affine $=$ Racomitrium affine (F. Weber et $\mathrm{D}$. Mohr) Lindb.

Racomitrium aquaticum = Racomitrium aquaticum (Brid. ex Schrad.) Brid.

Racomitrium canescens $=$ Racomitrium canescens (Hedw.) Brid.

Racomitrium heterostichum $=$ Racomitrium heterostichum (Hedw.) Brid.

Racomitrium lanuginosum = Racomitrium lanuginosum (Hedw.) Brid.

Rhabdoweisia fugax $=$ Rhabdoweisia fugax (Hedw.) Bruch et Schimp.

Rhizomnium punctatum = Rhizomnium punctatum (Hedw.) T. J. Kop.

Rhodobryum ontariense $=$ Rhodobryum ontariense (Kindb.) Kindb.

Rhodobryum roseum $=$ Rhodobryum roseum (Hedw.) Limpr.

Rhynchostegiella curviseta $=$ Rhynchostegiella curviseta (Brid.) Limpr.

Rhynchostegiella tenella $=$ Rhynchostegiella tenella (Dicks.) Limpr.

Rhynchostegiella teneriffae $=$ Rhynchostegiella teneriffae (Mont.) Dirkse et Bouman

Rhynchostegium confertum $=$ Rhynchostegium confertum (Dicks.) Schimp.

Rhynchostegium megapolitanum = Rhynchostegium megapolitanum (Blandow ex F. Weber et D.

Mohr) Schimp.

Rhynchostegium murale = Rhynchostegium murale (Hedw.) Schimp.

Rhynchostegium rotundifolium = Rhynchostegium rotundifolium (Scop. ex Brid.) Schimp.

Rhytidiadelphus squarrosus = Rhytidiadelphus squarrosus (Hedw.) Warnst.

Rhytidiadelphus triquetrus $=$ Hylocomiadelphus triquetrus (Hedw.) Ochyra et Stebel

Rhytidium rugosum $=$ Rhytidium rugosum (Hedw.) Kindb.

Saelania glaucescens $=$ Saelania glaucescens $($ Hedw.) Broth . 
Sanionia uncinata $=$ Sanionia uncinata (Hedw.) Loeske

Schistidium apocarpum $=$ Schistidium apocarpum (Hedw.) Bruch et Schimp.

Schistidium brunnescens subsp. brunnescens $=$ Schistidium brunnescens Limpr. subsp. brunnescens

Schistidium brunnescens subsp. griseum $=$ Schistidium brunnescens Limpr. subsp. griseum (Nees et Hornsch.) H. H. Blom

Schistidium confertum $=$ Schistidium confertum (Funck) Bruch et Schimp.

Schistidium confusum $=$ Schistidium confusum $\mathrm{H}$. H. Blom

Schistidium crassipilum $=$ Schistidium crassipilum $\mathrm{H}$. H. Blom

Schistidium dupretii = Schistidium dupretii (Thér.) W. A. Weber

Schistidium elegantulum $=$ Schistidium elegantulum $\mathrm{H}$. H. Blom

Schistidium flaccidum = Schistidium flaccidum (De Not.) Ochyra

Schistidium helveticum $=$ Schistidium helveticum (Schkuhr) Deguchi

Schistidium lancifolium $=$ Schistidium lancifolium (Kindb.) H. H. Blom

Schistidium papillosum $=$ Schistidium papillosum Culm.

Schistidium platyphyllum $=$ Schistidium platyphyllum (Mitt.) H. Perss.

Schistidium pruinosum $=$ Schistidium pruinosum (Wilson ex Schimp.) G. Roth

Schistidium robustum $=$ Schistidium robustum (Nees et Hornsch.) H. H. Blom

Scleropodium purum = Pseudoscleropodium purum (Hedw.) M. Fleisch.

Scorpidium scorpioides $=$ Scorpidium scorpioides (Hedw.) Limpr.

Seligeria calcarea $=$ Seligeria calcarea $($ Hedw.) Bruch et Schimp.

Seligeria campylopoda $=$ Blindiadelphus campylopodus (Kindb.) Fedosov et Ignatov

Seligeria donniana = Seligeria donniana (Sm.) Müll. Hal.

Seligeria pusilla $=$ Seligeria pusilla (Hedw.) Bruch et Schimp.

Seligeria recurvata $=$ Blindiadelphus recurvatus (Hedw.) Fedosov et Ignatov

Seligeria trifaria var. longifolia = Seligeria trifaria (Brid.) Lindb. var. longifolia (Lindb. ex Broth.)

Ochyra et Gos

Sphagnum angustifolium = Sphagnum angustifolium (C. E. O. Jensen ex Russow) C. E. O. Jensen

Sphagnum capillifolium = Sphagnum capillifolium (Ehrh.) Hedw.

Sphagnum centrale = Sphagnum centrale C. E. O. Jensen

Sphagnum compactum = Sphagnum compactum Lam. et DC.

Sphagnum contortum = Sphagnum contortum Schultz

Sphagnum cuspidatum = Sphagnum cuspidatum Ehrh. ex Hoffm.

Sphagnum denticulatum = Sphagnum auriculatum Schimp.

Sphagnum fallax = Sphagnum fallax (H. Klinggr.) H. Klinggr.

Sphagnum fimbriatum = Sphagnum fimbriatum Wilson

Sphagnum flexuosum = Sphagnum flexuosum Dozy et Molk.

Sphagnum girgensohnii = Sphagnum girgensobnii Russow

Sphagnum inundatum = Sphagnum inundatum Russow

Sphagnum magellanicum auct. p. p. = Sphagnum divinum Flatberg et Hassel

Sphagnum obtusum = Sphagnum obtusum Warnst.

Sphagnum palustre $=$ Sphagnum palustre $\mathrm{L}$.

Sphagnum platyphyllum = Sphagnum platyphyllum (Lindb. ex Braithw.) Warnst.

Sphagnum quinquefarium = Sphagnum quinquefarium (Braithw.) Warnst.

Sphagnum riparium = Sphagnum riparium Ångstr.

Sphagnum russowii = Sphagnum russowii Warnst.

Sphagnum squarrosum = Sphagnum squarrosum Crome

Sphagnum subnitens = Sphagnum subnitens Russow et Warnst.

Sphagnum subsecundum = Sphagnum subsecundum Nees 
Sphagnum teres $=$ Sphagnum teres (Schimp.) Ångstr.

Sphagnum warnstorfii = Sphagnum warnstorfi Russow

Splachnobryum obtusum = Splachnobryum obtusum (Brid.) Müll. Hal.

Taxiphyllum densifolium $=$ Taxiphyllum densifolium (Lindb. ex Broth.) Reimers

Taxiphyllum wissgrillii = Taxiphyllum wissgrillii (Garov.) Wijk et Margad.

Tetraphis pellucida $=$ Tetraphis pellucida $\mathrm{Hedw}$.

Thamnobryum alopecurum = Thamnobryum alopecurum (Hedw.) Gangulee

Thuidium abietinum = Abietinella abietina (Hedw.) M. Fleisch.

Thuidium delicatulum = Thuidium delicatulum (Hedw.) Schimp.

Thuidium philibertii = Thuidium assimile (Mitt.) A. Jaeger

Thuidium recognitum $=$ Thuidium recognitum (Hedw.) Lindb.

Thuidium tamariscinum = Thuidium tamariscinum (Hedw.) Schimp.

Timmia austriaca $=$ Timmia austriaca Hedw.

Timmia bavarica $=$ Timmia bavarica Hessl.

Tomentypnum nitens $=$ Tomentypnum nitens (Hedw.) Loeske

Tortella bambergeri auct. p. p. = Tortella fasciculata (Culm.) Culm.

Tortella bambergeri auct. p. p. = Tortella pseudofragilis (Thér.) Köckinger et Hedenäs

Tortella inclinata $=$ Tortella inclinata (R. Hedw.) Limpr.

Tortella tortuosa $=$ Tortella tortuosa (Hedw.) Limpr.

Tortula atrovirens $=$ Tortula atrovirens $(\mathrm{Sm}$.) Lindb.

Tortula brevissima $=$ Tortula brevissima Schiffn.

Tortula calcicolens $=$ Syntrichia calcicola J. J. Amann

Tortula caninervis subsp. spuria $=$ Syntrichia caninervis Mitt. var. gypsophila (J. J. Amann ex G.

Roth) Ochyra

Tortula crinita p. p. = Syntrichia montana Nees var. montana

Tortula inermis $=$ Tortula inermis (Brid.) Mont.

Tortula laevipila $=$ Syntrichia laevipila Brid.

Tortula latifolia $=$ Syntrichia latifolia (Bruch ex Hartm.) Huebener

Tortula mucronifolia = Tortula mucronifolia Schwägr.

Tortula muralis p. p. = Tortula muralis Hedw.

Tortula norvegica $=$ Syntrichia norvegica $\mathrm{F}$. Weber

Tortula obtusifolia $=$ Tortula muralis Hedw. subsp. obtusifolia (Schwägr.) Culm.

Tortula papillosa = Syntrichia papillosa (Wilson) Jur.

Tortula papillosissima p. p. = Syntrichia subpapillosissima (Bizot et R. B. Pierrot ex W. A. Kramer)

M. T. Gallego et J. Guerra

Tortula ruraliformis $=$ Syntrichia ruraliformis (Besch.) Mans.

Tortula ruralis p. p. = Syntrichia ruralis (Hedw.) F. Weber et D. Mohr var. ruralis

Tortula schimperi $=$ Tortula schimperi $\mathrm{M}$. J. Cano, O. Werner et J. Guerra

Tortula subulata $=$ Tortula subulata Hedw.

Tortula virescens $=$ Syntrichia virescens $($ De Not.) Ochyra

Trichostomum brachydontium $=$ Trichostomum brachydontium Bruch

Trichostomum crispulum $=$ Trichostomum crispulum Bruch

Ulota bruchii = Ulota bruchii Hornsch. ex Brid.

Ulota coarctata $=$ Ulota coarctata (P. Beauv.) Hammar

Ulota crispa p. p. = Ulota crispa (Hedw.) Brid.

Ulota hutchinsiae $=$ Ulota hutchinsiae $(\mathrm{Sm}$.) Hammar

Warnstorfia exannulata $=$ Sarmentypnum exannulatum (Schimp.) Hedenäs

Weissia brachycarpa $=$ Weissia brachycarpa (Nees et Hornsch.) Jur. 
Weissia condens $a=$ Weissia condensa (Voit) Lindb.

Weissia controvers $=$ Weissia controvers $a$ Hedw. var. controversa

Weissia fallax $=$ Weissia controversa Hedw. var. crispata (Nees et Hornsch.) Nyholm Weissia longifolia $=$ Weissia longifolia Mitt.

Weissia rostellata $=$ Weissia rostellata (Brid.) Lindb.

Weissia rutilans $=$ Weissia rutilans (Hedw.) Lindb.

Zygodon rupestris $=$ Zygodon rupestris Schimp. ex Lorentz 